\title{
Biological Effectiveness of Neutron Irradiation on Animals and Man
}

\author{
Tore Straume \\ (Ph.D. Thesis)
}

Manuscript date: November 1982

\section{MOTICE}

PORTIONS OF THIS REPORT ARE ILLEGIBLE. It

has been reproduced froni the best available copy to permit the brosdest possibie avaitability.

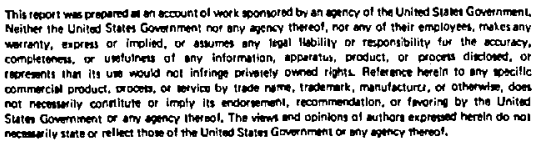


Blological Effectiveness of Neutron Irradiation on Animals and Man

\section{By}

TORE STRAUME

B.S. (University of Washington) 1973

M.S. (University of Washington) 1976

\section{DISSERTATION}

Submitted in partial satisfaction of the requirements for the degree of DOCTOR OF PHILOSOPHY

in

Blophyeles

in the

GRADUATE DIVISION

of the

UNIVERSITY OF CALIFORNIA

DAVIS

Approved:

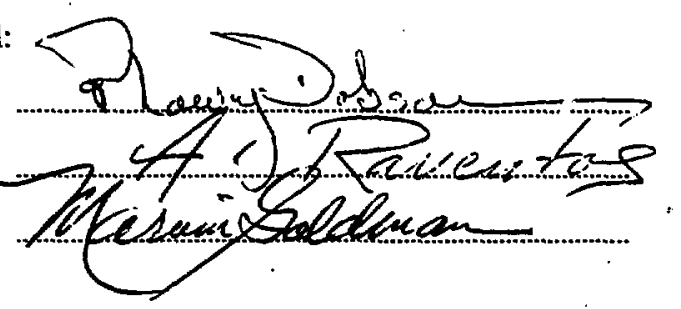

Committee in Charge

Deposited in the University Library. 
List of Figures

List of Tables . . . . . . . . . . . . . . . ix

Acknowledgement . . . . . . . . . . . . . . xiij

Abstract ..... . . . . . . . . . . . . $x v$

1. Introduction . . . . . . . . . . . . . . . 1

2. A Review of the Neutron RBE Literature . . . . . . . 7

The Comparison Radiation . . . . . . . . .8

Acute Radiation Mortality . . . . . . . . 10

Cell killing . . . . . . . . . . . 10

Carcinogenesis . . . . . . . . . . . 16

Mutagenesis . . . . . . . . . . . . . . . 19

Cataractogenesis . . . . . . . . . . . 31

Life Shortening • . • . . . . . . . . . . 31

Effect of Neutron Energy on RBE . . . . . . . 34

3. Neutron and Gamma-Ray Experiments on Mouse Oocytes . . . 39

Materials and Methods . . . . . . . . . . . 41

Results . . . . . . . . . . . . 51

Discussion . . . . . . . . . . . 72

Conclusions . . . . . . . . . . . . . 79

4. The Radiosensitive Target in Mouse Oocytes . . . . . 81

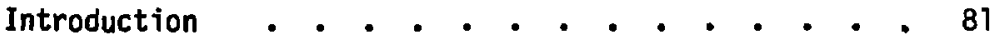

Target Size Estimation with Neutrons . • . . . 83

Apparent Inconsistencies . . . . . . . . . 85

Reconciliations . . . . . . . . . . . 89

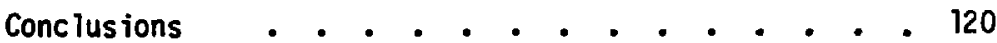

5. A Synthesis of the Human and Animal Neutron RBE Data . . 122

Human Neutron RBEs . • . . . . . . . . 122

Animal and Human Neutron RBEs Compared . . . . . 128

Implications for Radiological

Heal th Protection. . . . . . . . . . 133

6. Summary and Conclusions . . . . . . . . . . 147

Factors Affecting Neutron RBE . . . . . . . . 147 
Effects of Neutrons on Mouse Oocytes . . . . . 149

Effectiveness of Neutrons in Man . . . . . . . 152

References . . . . . . . . . . . . . . . 157

Appendix 1. Implications of new Hiroshima and

Nagasaki Dose Estimates: Cancer

Risk and Neutron RBE . . . . . . . . 165

Appendix 2. Sensitivity of Hiroshima and

Nagasaki Epidemiological

Inferences to Dosimetric Parameters . . . 174

Appendix 3. Cancer Risks and Neutron RBEs

from Hiroshima and Nagasaki . . . . . . 193 
1.1. Theoretical dose-effect relationships for neutrons and gamma rays

2.1. Chromosome aberrations induced in human lymphocytes irradiated in vitro with neutrons, $x$ rays, or ${ }^{60}$ Co gamma rays

2.2. Variation in neutron RBE with neutron dose for cell killing . . . . . . . . . . . .

2.3. The influence of plant-seed water concentration on neutron RBE

2.4. Variation in neutron RBE with neutron dose for the induction of tumors

2.5. Radiation induction of ovarian tumors in two mouse strains

2.6. Variation of neutron RBE with neutron dose for mutagenesis . . . . . . . . . . . . 21

2.7. Dose-response curves for somatic mutations in Tradescantia stamen hairs

2.8. Neutron-induced mutations in mouse oocytes - . . . 27

2.9. Variation in neutron RBE with neutron dose for radiation-induced cataracts in mice . . . . . . 31

2.10. Neutron RBEs for life shortening and principal malignancies compared 
2.11. Variation of neutron RBE with neutron energy . . . 35

3.1. Variation of neutron RBE with neutron dose

for highly radiosensitive biological endpoints . . . . 38

3.2. Radiosensitivity of mouse oocytes as a function of the animal's age at exposure . . . . . . . . . 40

3.3. Positioning of mice during 0.43-MeV neutron exposures . . 43

3.4. Dose transmisston of $0.43-$ MeV neutrons through leucite phantoms of various thickness . . . . . . 44

3.5. The influence of lateral variations in mouse positioning on absorbed dose from $0.43-\mathrm{MeV}$ neutrons .45

3.6. Neutron energy spectrum from a very thin ${ }^{252}$ Cf source

3.7. Oocyte survival following 0.43-MeV neutrun irradiation of 14-day old mice . . . . . . . . 5

3.8. A comparison of oocyte survival following 0.43-MeV neutron irradiation of mice on the 11 th or 17 th day post conception or at 14 days of age . . . 53

3.9. A comparison of oocyte survival following irradiation with $0.43-\mathrm{MeV}$ neutrons or ${ }^{60} \mathrm{Co}$ garma rays . . . . . 57

3.10. RBE for oocyte killing with 0.43-MeV neutrons as a function of neutron dose . . . . . . . 58

3.11. Oocyte survival following ${ }^{252}$ Cf fission irradiation . 60 
3.12. A comparison of oocyte survival following irradiation with "pure" fission neutrons or ${ }^{60}$ Co gamma rays . . .

3.13. RBE for fission neutrons as a function of neutron dose . . . . . . . . . . . . 64

3.14. The estimated effect of fission neutrons, fission gamma rays, and neutron-gamma-ray interactions on oocyte killing

3.15. Oocyte survival following $15-\mathrm{MeV}$ neutron irradiation of mice at 18 days of age . . . . . . 68

3.16. A comparision of oocyte survival following irradiation with 15-MeV neutrons or ${ }^{60}$ Co gamma rays . . . . . 69

3.17. Variation in neutron RBE with neutron energy for mouse oocyte killing . . . . . . . . . . 73

3.18. Variation in neutron RBE with the animal's age at exposure for mouse oocyte killing . . . . . . . . 74

3.19. Neutron RBEs for mouse oocytes compared to RBEs for other radiosensitive biological systems . . . . 76

4.1. Event frequency for various radiations as a function of tissue sphere diameter . . . . . . . . . 80

4.2. Dose-average of the specific energy imparted by single ${ }^{60}$ Co gama-ray events in tissue-equivalent spheres . . 86

4.3. Electron micrograph of an immature mouse oocyte . . 88

4.4. Dimensions of proton recoil tracks traversing the plasma membrane . . . . . . . . . . . 92 
4.5. Extrapolation number estimated from the oocyte survival curve for acute ${ }^{60} \mathrm{Co}$ gamma rays . . . . . 95

4.6. Computational method for plasma membrane traversal by proton recoils $\left(\theta_{2} \leq 90^{\circ}\right)$. . . . . . . . . 100

4.7. Computational method for plasma membrane traversal by proton recoils $\left(\theta_{2}>90^{\circ}\right)$. . . . . . . . . . 101

4.8. A cross-sectional representation of an oocyte showing the regions from where a proton recoil can traverse the plasma membrane . . . . . . 105

4.9. Model arrangement of $3_{\mathrm{H}}$ beta disintegrations near the plasma membrane . . . . . . . . . . 110

4.10. Computational method for plasma membrane traversal by ${ }^{3} \mathrm{H}$ beta particles

4.11. The number of ${ }^{3} \mathrm{H}$ beta particles traversing the plasma membrane as a function of average deposited energy 


\section{LIST OF TABLES}

2.7. Neutron RBE at the $\mathrm{LD}_{50}$ for acute radiation mortality . . . . . . . . . . . . 11

2.2. Selected neutron RBE studies on cell killing . . . . 12

2.3. Selected neutron RBE studies on experimental carcinogenesis . . . . . . . . 16

2.4. Selected neutron RBE studies on experimental mutagenesis . . . . . . . . 20

2.5. RBE values for induction of specific locus and dominant mutations in mouse spermatogonia by fission neutrons . . . . . . . 26

2.6. Specific locus mutations after exposure of mouse oocytes to fission neutrons . . . . . . 26

2.7. Representative neutron RBE studies on experimental cataractogenes is

2.8. Effect on neutron RBE of varying neutron energy . • . 34

3.1. Resu?ts from very-low-dose neutron RBE studies . . . . 37

3.2. Neutron and gamma-ray dose contributions from ${ }^{252}$ Cf source

3.3. Effect of $0.43-\mathrm{MeV}$ neutron exposure of mice at 14 days of age 
3.4. Effect of 0.43-MeV neutron exposure on the 17 th day postconception

3.5. Effect: of $0.43-\mathrm{MeV}$ neutron exposure on the 11 th day postconception

3.6. Results for dose-rate experiments with ${ }^{60}$ Co gamma rays . . . . . . . . . . . . 56

3.7. Effect of Californium-252 exposure of 14-day-old mice . . . . . . . . . . . . 59

3.8. Effect of Californium-252 exposures at several dose-rates . . . . . . . . . . .

3.9. Estimated effect of pure fission neutron exposure . . . 62

3.10. Effect of neutron and gamma-ray synergisms for ${ }^{252} \mathrm{Cf}$ exposures . . . . . . . . . . 65

3.11. 15-MeV neutron exposure at 18 days of age...+.67

4.1. Estimated radiosensitive target diameters in mouse oocytes . . . . . . . . . . . . . . 82

4.2. Variation in target diameter with mouse age for $0.43-\mathrm{MeV}$ neutrons . . . . . . . . . 82

4.3. Average energy deposition at $\mathrm{D}_{37}$ in the nucleus and total cell for the neutron radiations used

4.4. Oocyte target size estimates for the radiations used in this study 
4.5. Energy transfer characteristics of the

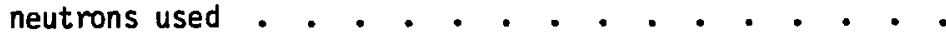

4.6. Track dimensions of recoil protons . . . . . . . . 91

4.7. Number of recoil proton cell traversals

required for oocyte $\mathrm{killing}$ by $15-\mathrm{MeV}$ neutrons, estimated by 3 methods

4.8. Diffusion distances for radiolys is products of water following ${ }^{60}$ Co irradiation . . . . . . 94

4.9. Number of ionizations ("hits") required in volume of plasma membrane for nocyte killing by ${ }^{60}$ Co gamma rays, estimated by three methods . . . . 96

4.10. Energy imparted to plasma membrane at $D_{37}$ by the radiations used . . . . . . . . . . . .

4.11. Solid angles for plasma membrane traversal by proton recoils originating at location $x, y, z$. . 104

4.12. Probabilities for plasma membrane traversa: and cell hit by a 0.215 -Mev recoil proton . . . . . 108

4.13. Number of ${ }^{3} \mathrm{H}$ beta rays traversing plasma membrane at $\mathrm{D}_{37}$ as a function of energy deposited . . 113

4.14. Effect of ${ }^{3} \mathrm{H}$ beta ray expostre on mouse oocytes . . 117

4.15. Evidence for plasma membrane as the radiosensitive target in mouse oocytes 
5.1 Ratios of carcinogenic efficiency for Hiroshima and Nagasaki radiation (using new Jose estimates) . . . 123

5.2. Neutron RBE est imates for human radiation effects . . 124

5.3. Human sensitivity to cancer induction by low-LET radiation and neutron RBE compared . . . . . . . 125

5.4. Animal and human neutron RBE data compared . . . . 126

5.5. Risk-coefficients derived by Method 2 . . . . . . 137

5.6. LOW-LET risk coefficients at low doses

(10 rad) estimated directly from

linear-quadratic curve fitting . . . . . . 140

5.7. Low-LET risk coefficients at low doses estimated from high doses $(200 \mathrm{rad}) . . .40$

5.8. Neutron risk coefficients for total malignancies derived by the four methods . . . . . 141

5.9. Neutron "quality factor" estimates derived by the four methods . . . . . . . . 143

5.10. Estimated uncertainties in Hiroshima and Nagasaki dosimetry and biological effects data . . . . . 144

6.1. A ranking of factors according to their ability to affect neutron RBE . . . . . 148

6.2. Summary of neutron RBEs for human effects . . . . . 152 
I would like to thank Drs. R. L. Dobson, M. L. Mendelsohn, D. Moore, E. Goldberg, Ms. T. C. Kwan and Mr. L. D. Stephens at the Lawrence Livermore National Laboratory; Ors. M. Goldman, A. Raventos, D. Deamer, and J. Jungerman of the University of California at Davis; Drs. N. Rohrig, D. Joel, Mr. S. Marino and Mr. R. Mills at the Brookhaven National Laboratory. 


\section{ABSTRACT}

Neutron experiments on a highly radiosensitive in vivo system -- oocytes in mice -- provide new insight into the nature of the radiosensitive targets of these important cells. With the radiobiological literature as background, neutron data from animais and humans (including studies by the author using reevaluated Hiroshima-Nagasaki dosimetry) are integrated, and the controversial question of radiation protection standards for neutrons is addressed. 0ocyte killing in juvenile mice by $0,43-\mathrm{MeV},{ }^{252} \mathrm{Cf}-\mathrm{fission}$, and $15-\mathrm{MeV}$ neutron:i, compared with that by ${ }^{60} \mathrm{Co}$ gamma rays, yields unusually low neutron RBEs (relative biological effectiveness). At 0.1 rad of $0.43-M e V$ neutrons the RBE is only 1.8 , contrasting greatly with values of 100 or more reported at low-doses for other endpoints. In mice just prior to birth, however, when oocytes are less radiosensitive, the neutron RBE is much higher, similar to values for most other mammalian endpoints. This dramatic change in neutron RBE with mouse age (occurring within 2-3 days) can be explained as the result of a shift from a less radiosensitive target (presumably nuclear ONA) to a much more radiosensitive one (probably the oocyte plasma membrane).

Study of the neutron literature reveals that factors most important in influencing neutron RBE are dose and biological endpoint. For most (but not all) endpoints neutron RBE increases 
with decraasing dose. Effects of other factors (neutron energy, dose rate, etc.) must therefore be evaluated at constant dose (rather than constant level of effect as generally done). Critical analysis of published mutation data shows that the influence of neutron energy on RBE has frequentiy been overestimated.

Both animal and human data suggest the intriguing general pattern (albeit with exceptions) of low neutron RBEs for malignancies. that have known (or suspected) viral association (e.g., Iymphomas) and high RBEs (similar to those for chromosome aberrations) for malignancies not so associated (e.g., lung cancer).

Using various approaches, a value for the neutron Quality Factor (Q, a radiation protection standard) is estimated as $17( \pm 100 \%)$, much lower than 100 which has been suggested. With the large uncertainty, 17 is not markedly different from the value of 10 presently in general use. 
1. INTRODUCTION

All ionizing radiations are not equally damaging to living things. Differences in the submicroscopic distribution patterns of energy that differing radiations transfer to matter can profoundly. influence their biological effectiveness. Ionizing radiations, whether they are purely electromagnetic or consist of subatomic particles, display energy transfer characteristics that can often be used to distinguish them. Neutron radiation, which is our principal concern here, transfers energy through collisions with atomic nuclei, and the resulting recoil ions produce dense tracks of ionization as they dissipate their kinetic energies. Gamma rays, on the other hand, transfer their energy to atomic e?ectrons, which are much less densely ionizing. Linear Energy Transfer (LET) is a measure of this ionization density and is defined as the average energy lost per unit of distance traversed by the recoil particle; it is generally expressed in $\mathrm{keV} / \mu \mathrm{m}$. The concept of LET is useful in comparing the biological effectiveness of various radiations, and it provides an important basis for the quantitative analyses presented here.

The effectiveness of any ionizing radiation may be compared to that of a "standard" radiation (conventionally 250-kVp $\times$ rays or gamna rays) by the ratio of doses that produce equal levels of effect. This ratio is called the Relative Biological Effectiveness (RBE) and is defined as:

$$
\mathrm{RBE}=\frac{\text { dose of "standard" comparison radiation }}{\text { dose of radiation of interest }} .
$$


The RBE provides an index for the relative potencies of different radiations in producing biological damage and is, therefore, useful in many areas of radiation-effects analysis, e.g., in establishing radiological health protection standards, in improving tumor therapeutic procefures, and as a tool in basic biological research.

The present work is concerned with neutron RBE in both a fundamental and practical sense. An attempt is made to uncover basic factors that influence the neuiron RBE and to determine how these factors may vary in different biological systems. It is believed that by focusing on the principal factors affecting RBE a better basis can be provided for its practical use.

A practical problem, which presently is receiving much attention, concerns the adequacy of radiological health protection standaris. Knowing the effectiveness of radiation is essential to the development of such standards, which should provide consistent dose limits for all radiation types. This requires (ideally) that dose-response curves be determined for the biological effects of principal concern to human health, using a variety of radiations and values of LET. Dose-response curves for many effects in man can be obtained from survivors of the Hiroshima and Nagasaki atomic bombings, from persons exposed medically, from those exposed occupationally, and from the few individuals exposed in radiation accidents. Since the focus here is primarily on neutrons and associated RBE values, the relevant human data base is narrowed to only the Japanese A-bomb survivors and a few nuclear criticality 
accident cases, as they alone received nearly uniform whole body exposures to significant amounts of neutrons.

Although large efforts have been underway to gather and analyze these data, only for a few human health erfects do they appear of sufficient statistical robustness to allow construction of meaningful dose-response curves. These effects include leukemia, breast cancer, lung cancer, chromosome aberrations, and all malignancies combined. Confidence in these dose-response curves depends, of course, upon the quality of both the biomedical data and the dosimetry. For the effects of greatest interest here (rare events, such as malignancies and mutations), the biomedical data are generaliy assumed to be Poisson distributed hence confidence intervals can be obtained by simple statistical procedures. The dosimetry data, however, for in vivo human exposures are gererally retrospective and likely to have considerable (and often difficult to quantify) uncertainty. At the present time the Japanese A-bonb dosimetry data are being reevaluated, and biomedical inferences drawn from them must be accepted only with caution. In light of both the lack of human dose-response data for, numerous important effects and the considerable uncertainties in the data where dose-response curves can be constructed, it is desirable to supplement our knowledge with animal experiments. The present thesis attempts to bring together the human and animal neutron-effects data, with the hope that 
similarities and differences will further our understanding of basic mechanisms of radiation action and provide new insight into the relevancy of extrapolation from animals to man.

Many factors are known to affect neutron RBE, including dose, dose rate, radiation energy, LET, chemical environment of the energy absorption event, and the biological system being studied. Some of these factors appear to influence RBE in much the same way regardless of biological endpoint, For example, RBE usually increases with decreasing dose and seems, in general, to follow a rather predictable trend that can often (but not always) be described by the function $R B E=k D_{n}^{-1 / 2}$, where $k$ is a constant and $D_{n}$ is the neutron dose (1). It has been postulated that the reason RBEs for so many endpoints follow this function is because these radiation effects are induced tinrough mechanisms conforming with the Theory of Dual Radiation Action (2), which states that two sublesions must combine to form a lesion before biological damage can be realized. The result of these mechanisms, at doses of concern here, is tinat neutrons of moderate energy will yield linear dose-response curves, while those for garma rays will be linear-quadratic. This is illustrated in Fig. 1.1 on a $\log -\log$ plot. It can be seen that neutron RBE (the ratio of equal-effect doses) will increase with decreasing dose, but at very low doses it will become constant as the gamma-ray curve also becomes linear (as slope approaches 1 on $\log -\log$ plot). It will be pointed out in Chapter 2 that although many endpoints appear to be consistent with this theory there are also numerous (and important) exceptions. 


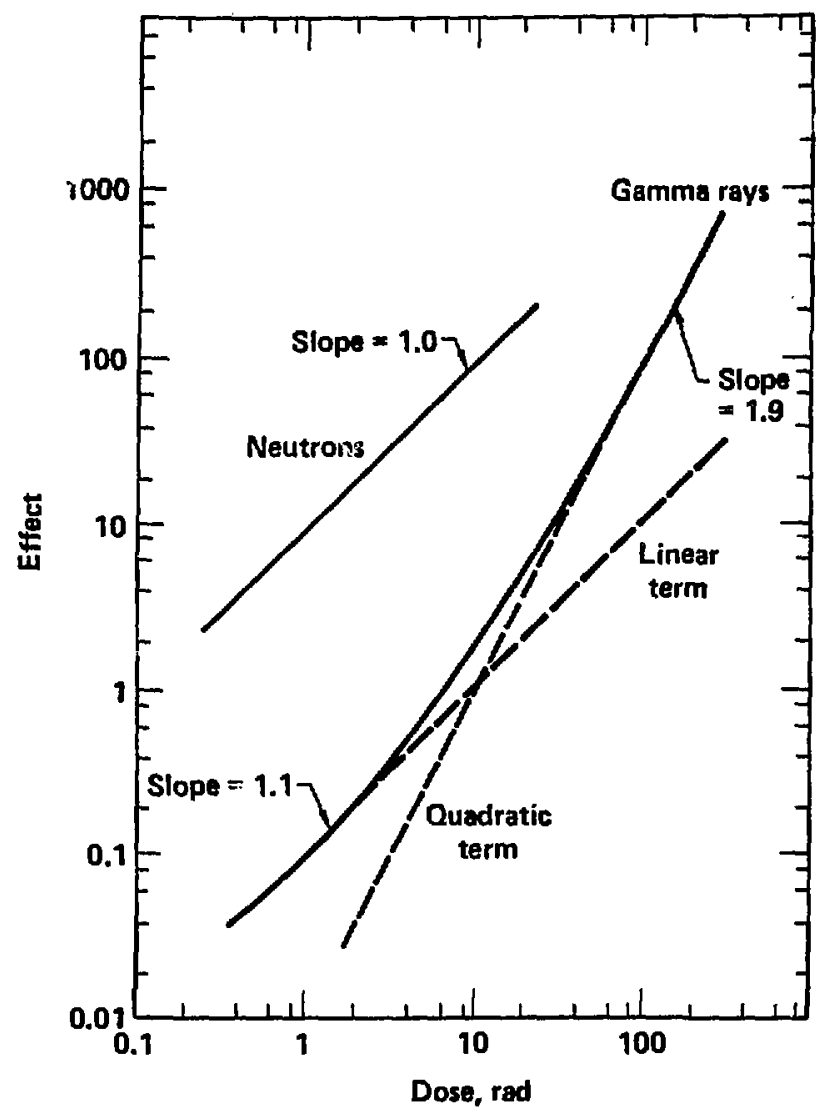

FIG. 1.1. Theoretical dose-effect relationships for neutrons and garma rays. The linear function for neutrons and the linear-quadratic function for gamma rays are illustrated. The $10 \mathrm{~g}-\mathrm{log}$ plot allows simple visualization of linear and quadratic components. A line with slope of 1 on a log-log plot is a linear function and one with a slope of 2 is a quadratic function. Madified from (3). 
A handful of investigators have published reports during the past decade showing that neutron RBEs may reach very higin values at: low doses. Their experiments, using highly sensitive indicators of radiobiological damage (e.g., somatic mutations in Tradescantia stamen hairs $(4,5)$, mammary neoplasms in rats $(6)$, and lens opacities in mice $(7,8))$, have demonstrated that neutron RBEs at very low doses (less than 1 rad of neutrons) can reach values in excess of 100 . Only a few biological endpoints are sufficiently radiosensitive to allow direct neutron RBE measurement in the one-rad range; it is important that they be identified and thoroughly studied. One of the most radiosensitive mammalian endpoints known, oocyte killing in juvenile mice, has not been adequately studied with neutron irradiation and was selected as the experimental endpoint here.

The principal objectives of the present work are to 1) bring together the experimental and human neutron dose-effect data so that similarities and differences may be identified, thereby providing balanced insight into the biological effectiveness of neutron radiation; 2) uncover fundamental mechanisms of radiation action through basic radiobiological research; and 3) consider how these new insights may influence radiological health protection standards. 


\section{A REVIEW OF THE NEUTRON RBE LITERATURE}

A sery large number of studies on neutron RBE are reported in the literature. All of then cannot be dealt with here, so represeritative examples from each of several broad topics are selected to illustrate the present-day knowledge on this subject.

The neutron RBE literature can be dividad into three major categories. There are many reports oriented toward cancer radiotherapy, others principally interested in radiological health protection, and numerous ones inakill up a highly diverse radiobiological data base concerned with uncovering basic biological mechanisms (often without specific regard to either of the first two catagories). Although overlafping occurs, these are separate research areas requiring differing approaches. For tumor sterilization, doses of several thous and rad are required, whereas doses of most concern to radiological health protection are in the one-rad range. The focus of the present dissertation is on those basic factors that influence neutron RPF and how they may affect our understanding of human radiobiology. Special emphasis is placed on low doses. The literature pertaining to cancer radiotherapy will not be reviewed here, as this area of research is not directly considered in this thesis. The radiotherapy data have been previously reviewed by the author (9). 
The radiation chosen as a standard against which neutron dose-response data are compared to obtain RBEs wi11, of course, affect the resulting RBE values. By convention, the comparison radiation usually selected is $x$ rays or gamma rays. The energy and iritensity often depends upon the source most readily available to the experimenter. During the early days of radiobiology, $x$-ray generators were used almost exclusively, with peak voltages in the 200 to $300 \mathrm{kV}$ range. However, more recent interest in effects from chronically delivered radiation, for which these machines are not practical, has resulted in increased use of gamma-ray emitting sources, e.g., ${ }^{60} \mathrm{Co}$ and ${ }^{137} \mathrm{Cs}$, which allow low-dose-rate and long-term exposures.

Photon energy and dose rate of the comparison radiation can affect the neutron RBE. The influence of gamma-ray dose rate and photon energy on neutron RBE can be inferred from Fig. 2.1 for radiation-induced chromosome aberrations in human lymphocytes $(10,11)$. It is apparent that the neutron RBE decreases as the ${ }^{60}$ Co gamma-ray dose-rate increases and that $x$ rays (the curve shown is for $250-k V \times$ rays) yield lower neutron RBEs than do the higher energy (1.25-MeV) gamma rays. 


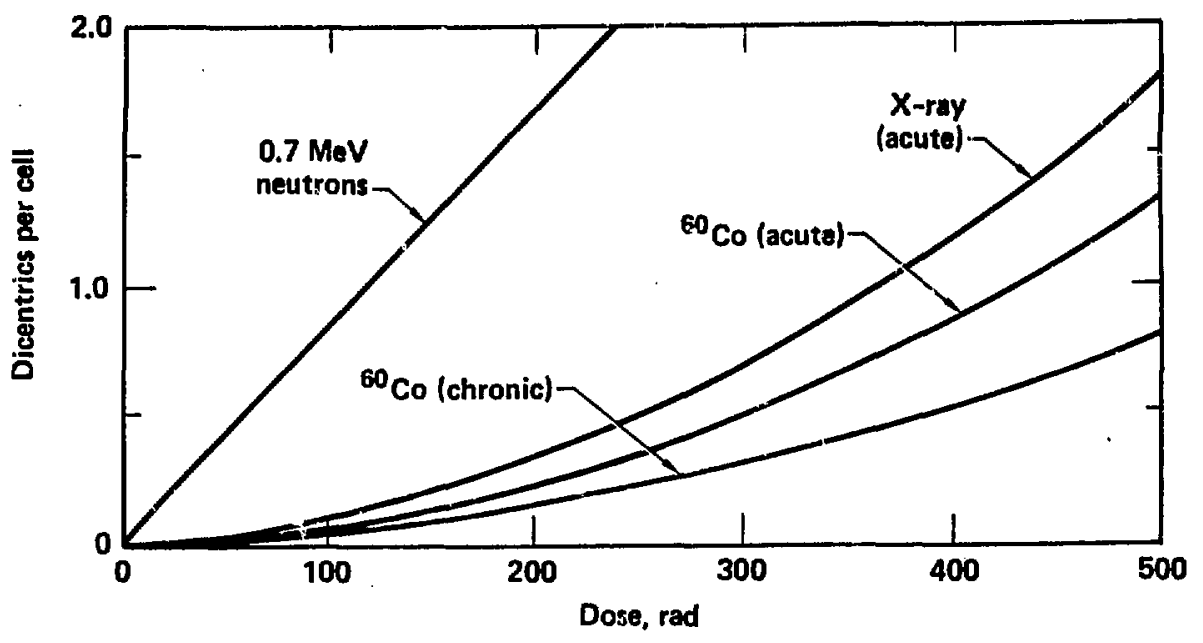

FIG. 2.1. Chromosome aberrations (dicentrics) induced in human iymphocytes irradiated in vitro with neutrons, $x$ rays, or $50 \mathrm{Co}$ gamma rays (see text). Modified from $(10,11)$. 
Death in aninals from large duses of radiation is generally divicied into three categories, the bone-marrow, gistrointestinal, and central-nervous-system syndromes, depending upon the organ systems primarily affected alıd responsible for mortality. RBES derived at the $L D_{50}$ (lethal dose for $50 \%$ of those exposed) for bone-marrow and gastrointestinal syndromes are listed in Table 2.1 as functions of neutron energy. For both enduoints neutron RBE increases with decreasing neutron energy. This phenomenon is observed for most endpoints, and is expected on microdosimetric grounds (see e.g. References 2,12-15). Also from Table 2.1 it can be seen that the intestinal syndrume yields larger neutron RBEs than Joes the bone-marrow syndrome, probably reilecting the relatively great $3 r^{\circ}$ sensitivity of the marrow cells to low-LET radiation. The observed neutron RBEs for these syndromes appear related (as expected) to the radiosensitivities of the target-cell populations (16).

\section{CELL KILLING}

Neutron RBEs for cell killing, derived for various neutron energies and doses, are listed in Table 2.2. The data that allow neutron RBE evaluation over a range of doses are shown in Fig. 2.2. They demonstrato the generally observed trend of increasing RBE with decreasing dose. It is also noted in Fig. 2.2 that RBE decreases 
TABLE 2.1. Neutron RBE at the $L_{50}$ for acute radiation mortality.

\begin{tabular}{lccccc}
\hline \multicolumn{2}{c}{ Radiation* } & $\begin{array}{c}\text { Neutron } \\
\text { dose } \\
\text { (rad) }\end{array}$ & RBE & $\begin{array}{c}\text { Biological } \\
\text { endpoint }\end{array}$ & Reference \\
\hline Neutrons & Photons & 384 & 1.9 & Bone-marrow & 16 \\
\hline Fission & $250-k V p \times$ rays & & & & \\
$8 \mathrm{MeV}$ & $250-k V p \times$ rays & 486 & 1.5 & syndrome in & 16 \\
$15 \mathrm{MeV}$ & $250-k V p \times$ rays & 670 & 1.1 & mice & 16 \\
& & & & & \\
Fission & $250-k V p \times$ rays & 450 & 3.0 & Intestinal & 16 \\
$8 \mathrm{MeV}$ & $250-k V P \times$ rays & 482 & 2.8 & syndrome in & 16 \\
$15 \mathrm{MeV}$ & $250-k V p \times$ rays & 964 & 1.4 & mice & 16 \\
& & & & & \\
\hline
\end{tabular}

* Whole-body exposures.

as neutron energy is increased from $0.43 \mathrm{MeV}$ to $14 \mathrm{MeV}$. Since significant cell killing (for most cell types) requires rather large doses, the largest RBEs attained in studies on cell killing (except for dry plant seeds) are about 10. However, larger values are suggested at lower doses (from slopes of curves in Fig. 2.2) and may be quantifiable by further experiments.

The high RBEs observed with dry plant seeds are drastically lowered by soaking the seeds in water prior to irradiation, see Fig. 2.3. As water content approximates those found in soft animal tissues (60-80\%), the plant-seed RBEs become similar to those observed for animal cell killing (Fig. 2.2). 
TABLE 2.2. Selected neutron RRE studies on cell killing.

\begin{tabular}{|c|c|c|c|c|c|}
\hline \multicolumn{2}{|c|}{ Radiation } & \multirow{2}{*}{$\begin{array}{l}\text { Neutron } \\
\text { dose } \\
\text { (rad) }\end{array}$} & \multirow[b]{2}{*}{ RBE } & \multirow{2}{*}{$\begin{array}{l}\text { Biological } \\
\text { endpoint }\end{array}$} & \multirow[b]{2}{*}{ Referen } \\
\hline Neutrons & Photons & & & & \\
\hline $0.43 \mathrm{MeV}$ & $250-k V p \times$ rays & $15-210^{\star}$ & $10-4^{*}$ & Hamster cell killing & 17 \\
\hline $\mathrm{MeV}$ & $250-k V p \times$ rays & $\Delta 00-1000$ & $5-1.8$ & Mouse skin damage & 18 \\
\hline $0.43 \mathrm{MeV}$ & ${ }^{60}$ Co gamma rays & $4-10$ & $10-6$ & Mouse spermatogoniai killing & 19 \\
\hline $1.8 \mathrm{MeV}$ & ${ }^{60}$ Co gamma rays & $4-13$ & $9-5$ & $n$ & 19 \\
\hline $\mathrm{MeV}$ & ${ }^{60}$ Co gamma rays & $10-30$ & $4-2$ & " & 19 \\
\hline $\mathrm{MeV}$ & $220-k V p \times$ rays & $7-21$ & 21 & Mouse occyte killing & 20 \\
\hline $\mathrm{MeV}$ & $250-k V p \times$ rays & 12 & 13.2 & $50 \%$ mouse testis weight loss & 21 \\
\hline $\mathrm{MeV}$ & $250-k V p \times$ rays & 40 & 3.8 & " & 21 \\
\hline $15 \mathrm{MeV}$ & $250-k V p \times$ rays & 75 & 2.3 & $"$ & 21 \\
\hline $400 \mathrm{MeV}$ & $250-k V p \times$ rays & 100 & 1.7 & $"$ & 21 \\
\hline $600 \mathrm{MeV}$ & $250-k V p \times$ rays & 80 & 2.4 & $n$ & 21 \\
\hline Fission & $250-k V p \times$ rays & 1020 & 57. & $\begin{array}{l}\text { Plant seedling growth reduction } \\
\text { (dry seeds irradiated) }\end{array}$ & 22 \\
\hline Fission & $250-k V p \times$ rays & 230 & 6.7 & $\begin{array}{l}\text { Plant seedling growth } \\
\text { reduction (wet seeds irradiate }\end{array}$ & 22 \\
\hline
\end{tabular}


TABLE 2.2. (Continued)

\begin{tabular}{llllll}
$22 \mathrm{MeV}$ & $137 \mathrm{Cs}$ gamma rays & $100-600$ & $2.9-2.2$ & Mouse testis stem-cell killing & 23 \\
$15 \mathrm{MeV}$ & $300-\mathrm{kVp} \times$ rays & 2500 & 2.2 & LD50 pulmonary lethality & 24 \\
$15 \mathrm{MeV}$ & $300-\mathrm{kVp} \times$ rays & 889 & 3.0 & $\begin{array}{l}\text { LD } \\
\text { (D) esophageal lethality }\end{array}$ & 24 \\
$8 \mathrm{MeV}$ & $250-\mathrm{kVp} \times$ rays & $12-500$ & $4.7-1.5$ & $\begin{array}{c}\text { Damage to hematopoietic } \\
\text { tissue }\end{array}$ & 18 \\
$8 \mathrm{MeV}$ & $250-\mathrm{kVp} \times$ rays & $60-800$ & $3.8-1.7$ & Damage to growing cartilage & 18 \\
$8 \mathrm{MeV}$ & $250-\mathrm{kVp} \times$ rays & $250-1500$ & $3.5-2.0$ & Damage to mouse intestine & 18 \\
\hline
\end{tabular}

* RBE values are obtained at the neutron doses given. For some endpoints RBE values are kncwn over a range of ratron doses. Here and in subsequent Tables, the first listed RBE value (of a range) corresponds to the lowest neutron dose evaluated and the second RBE value correspond to the highest neutron dose evaluated. 


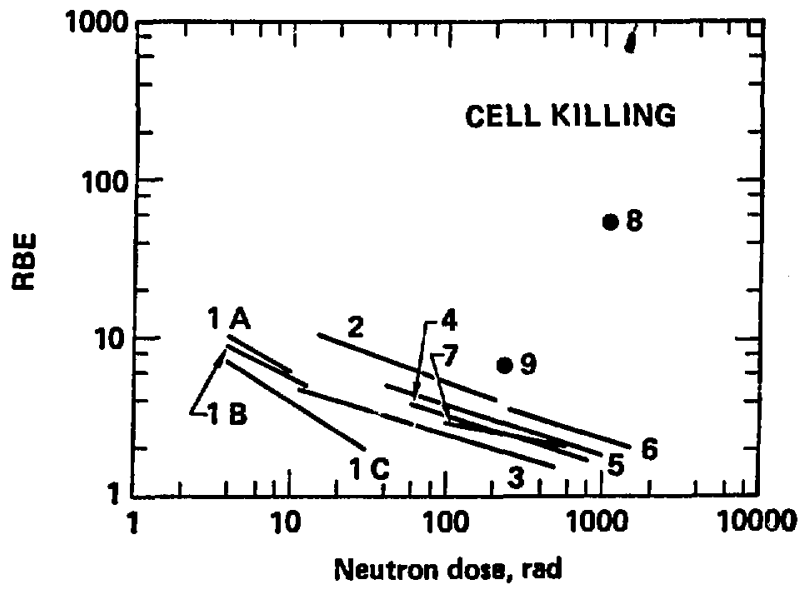

FIG. 2.2 Variation in neutron RBE with neutron dose for cell killing. Mouse spermatogonial cell killing with $0.43-\mathrm{MeV}$ neutrons (curve $1 A$ ), with 1.8-MeV neutrons (curve IB), and with 14-MeV neutrons (curve IC); V-79 hamster cell killing with $0.43-\mathrm{MeV}$ neutrons (curve 2); damage to haemopoietic tissue with $8-\mathrm{MeV}$ neutrons (curve 3 ); damage to growing cartilage with $8-M e V$ neutrons (curve 4); mouse skin damage with $8-\mathrm{MeV}$ neutrons (curve 5); damage to mouse intestine with 8-MeV neutrons (curve 6); mouse testis stem-cell killing with 22-MeV neutrons (curve 7); barley seedling growth reduction with fission neutrons seeds with $13 \%$ water content (curve 8) and with $85 \%$ water content (curve 9). 


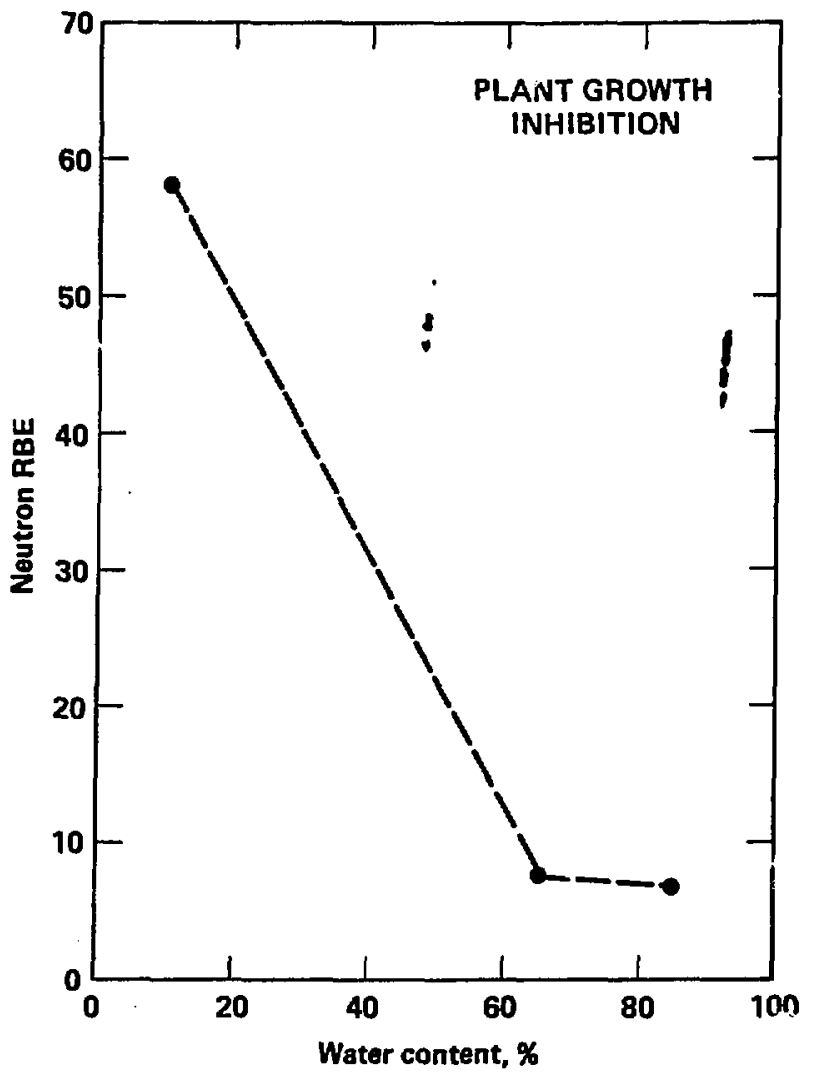

FIG. 2.3. The influence of plant-seed (barley) water concentration on neutron RBE as determined for plant growth inhibition using fission neutrons and $250 \mathrm{kVp} \times$ rays (from reference 22 ). 


\section{CARCINOGENESIS}

Neutron RBE studies on carcinogenesis are summarized in Table 2.3. The RBEs listed correspond to various neutron doses. Here however, in the case of neoplasia induction, the data indicate increasing RBE with decreasing dose for only some tumors; others yield RBEs not significantly different from unity, as seen in Fig. 2.4. This interesting departure from the RBE functions observed for cell killing suggests different mechanisms in the induction of at least some cancers and of cell lethality, and w1ll be discussed further in Chapter 5. It must be pointed out that sufficient uncertainties exist in most of the experimental carcinogenesis data so that the slopes of the RBE-vs-dose functions shown in Fig. 2.4 must be considered approximate only.

The neutron RBE function for mammary tumors in the Sprague-Dawley rat (Curve 1 in Fig. 2.4) is peculiar in that the observed RBE increase with decreasing dose does not result from the same underlying reasons as for other endpoints (29). The usual reason for an increasing neutron RBE with decreasing dose is that the neutron dose-effect curve is linear whereas the garma-ray dose-effect curve has a strong quadratic term. The rat mammary tumor data cannot be adequately fitted by these functions for neutrons and gamma rays and, in fact, do not appear to behave according to the Theory of Dual Radiation Action (29). The tumor incidence curve for neutrons shows negative curvature at very low doses (doses delivering much less than one energy absorption event per cell) while the gamma-ray curve is 
TABLE 2.3. Selected neutron RBE studies on experimental carcinogenesis.

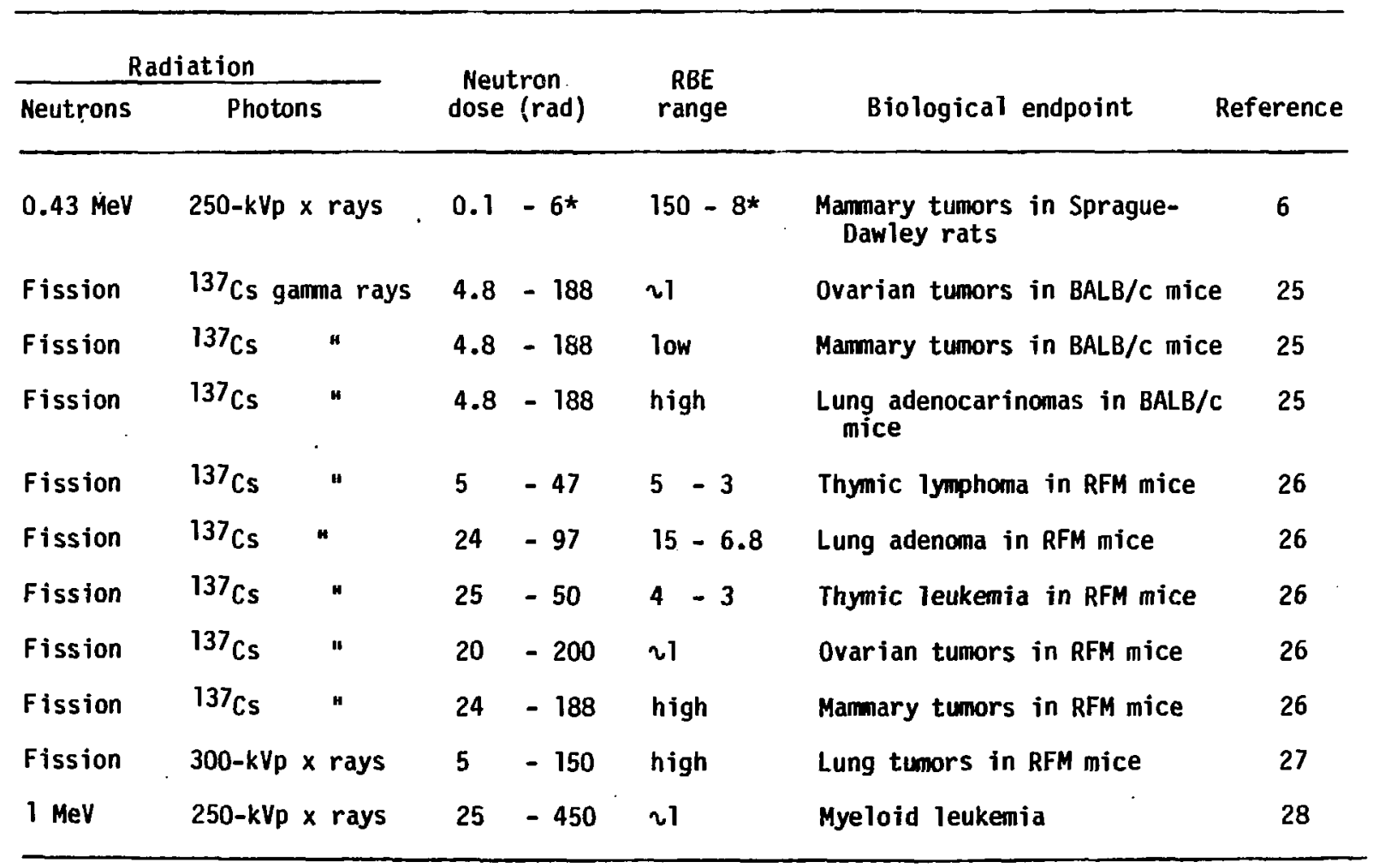

* See Table 2.2 footnote. 


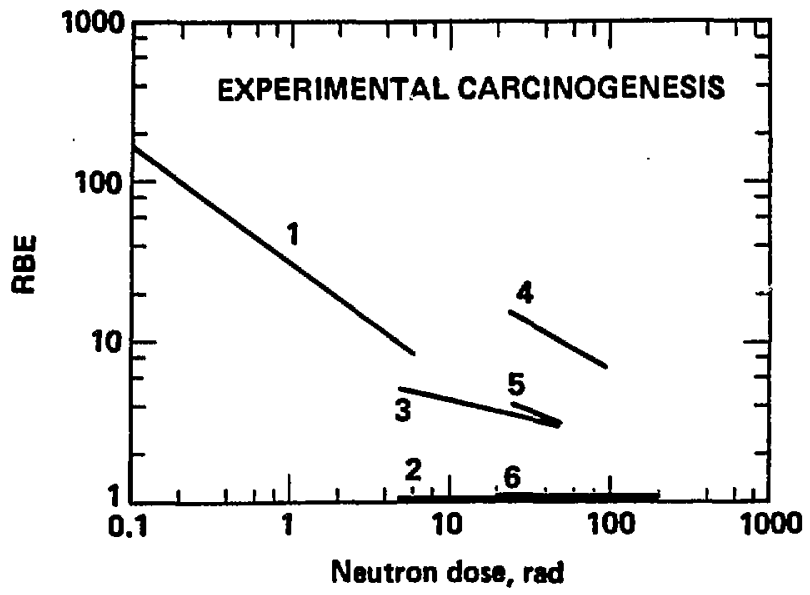

FIG. 2.4. Variation in neutron RBE with neutron dose for the induction of tumors. Rat mammary tumors with $0.43-\mathrm{MeV}$ neutrons (curve 1), ovarian tumors in mice with fission neutrons (curves 2 and 6), thymic lymphoma in mice with fission neutrons (curve 3), lung adenoma in mice with fission neutrons (curve 4), and thymic leukemia in mice with fission neutrons (curve 5 ). 
nearly linear. The net result is that the neutron RBE varies with dose as it does in other endpoints (Fig. 2.4), but for totally different reasons! In addition to peculiarities in dose-effect curves in Sprague-Dawley rats, the high spontaneous incidence of marmary tumors (the control and exposed animals show similar tumor incidence near the end of their life spans $(30$, implying that the tumor appearance is simply accelerated rather than induced) makes these data of questionable relevance to man.

The RBE for ovarian tumors in mice (curves 2 and 6 in Fig. 2.4) is strikingly low compared to those for other effects. Figure 2.5 shows replotted data for the induction of ovarian tumors in mice $(25,26)$. It is interesting to note the apparent dose-rate effect for neutrons. Neutrons delivered at low dose rate (chronically) appear much less effective than when given acutely. It is suggested here that ovarian tumors result from depletion (by radiation) of the mouse's oocyte supply (see Chapter 3) and that the previously noted neutron "dose-rate" effect $(25,26)$ is simply a result of the oocytes becoming more radioresistant with nouse age. An experiment is presently underway in this Laboratory to test this hypothesis.

\section{MUTAGENESIS}

A representative sample of neutron RBE studies on experimental mutagenesis is listed in Table 2.4 for various neutron energies and doses. Figure 2.6 illustrates how the experimentally determined RBEs vary with neutron dose, showing again that neutron RBE increases with decreasing dose. It is interesting to note that the RBE-VS-dose 


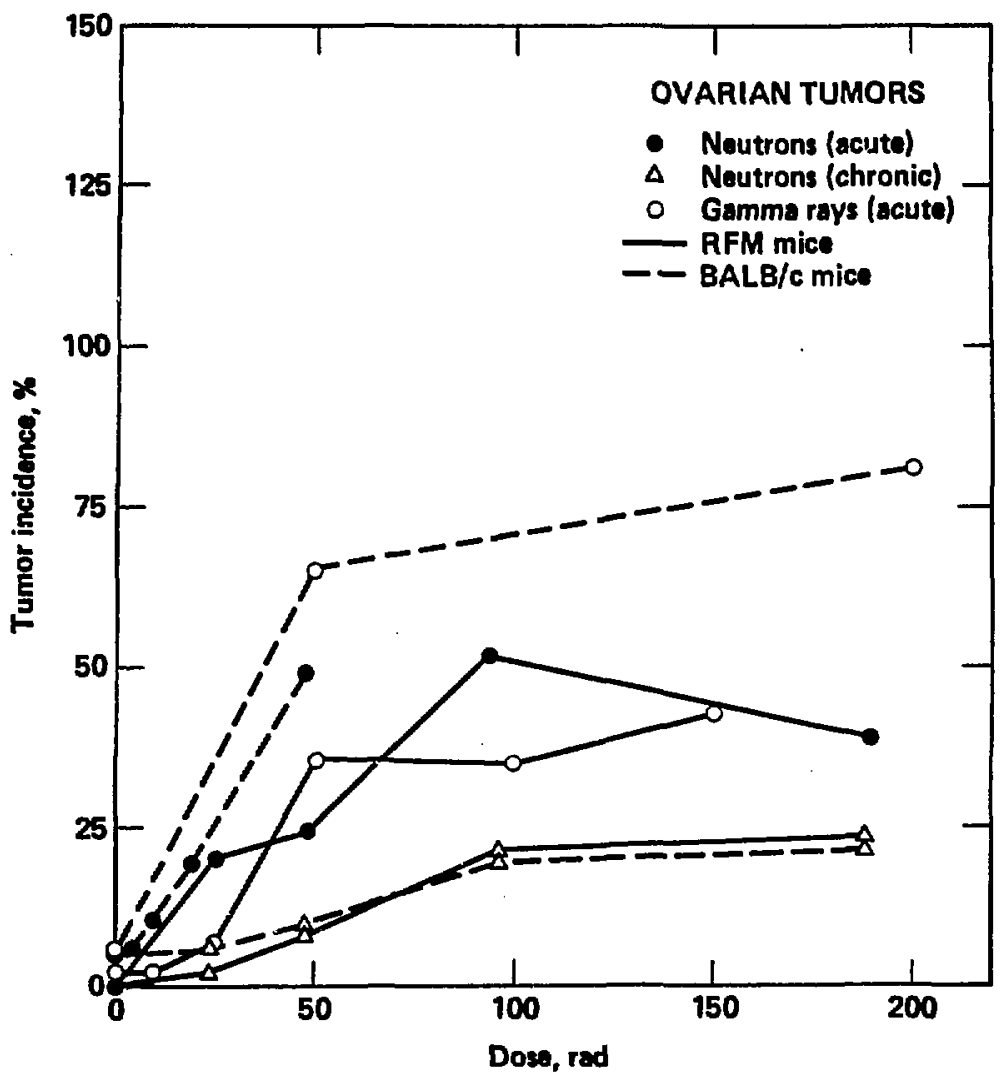

FIG. 2.5. Radiation induction of ovarian tumors in two mouse strains. Fission neutrons and $937 \mathrm{Cs}$ gamma rays were used. Platted from tabular data given by $(25,26)$. 
TABLE 2.4. Selected neutron RBE studies on experimental mutagenesis.

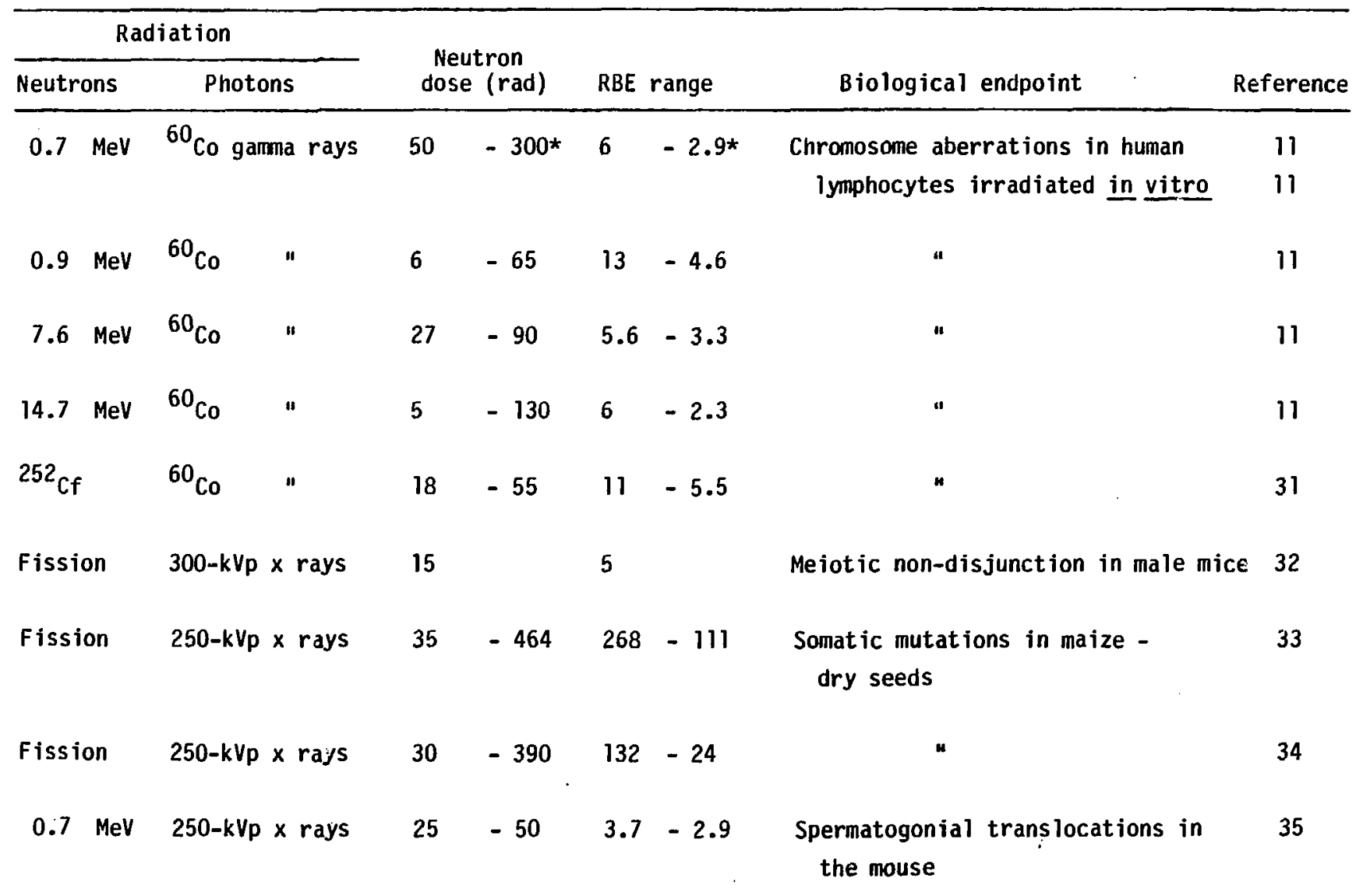


TABLE 2.4. (Cont inued)

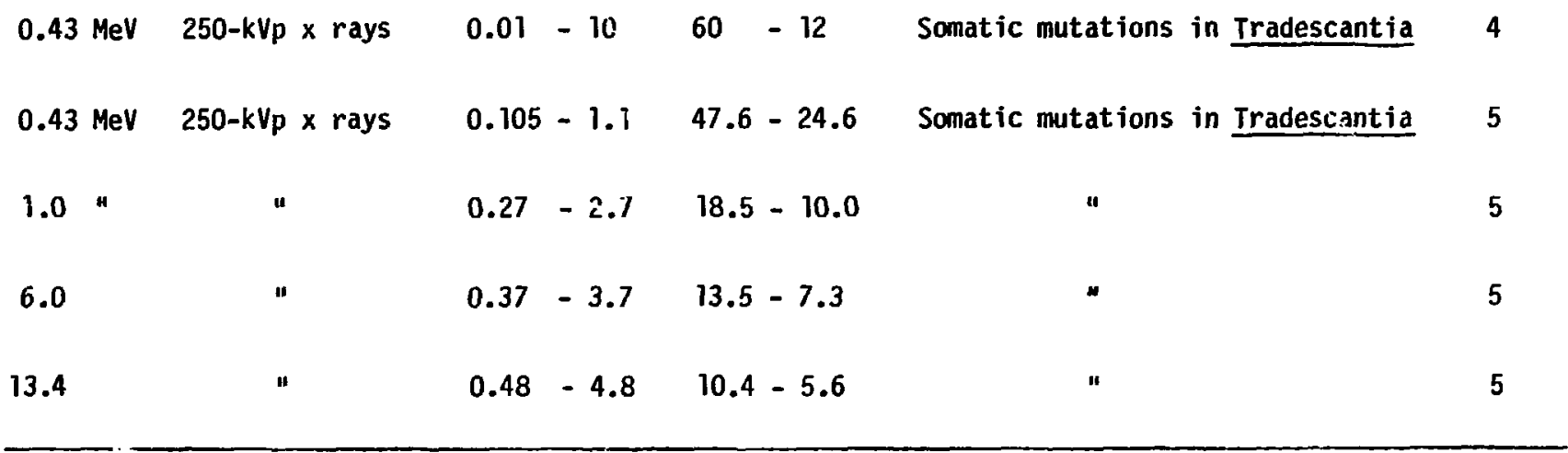

* See Table 2.2 


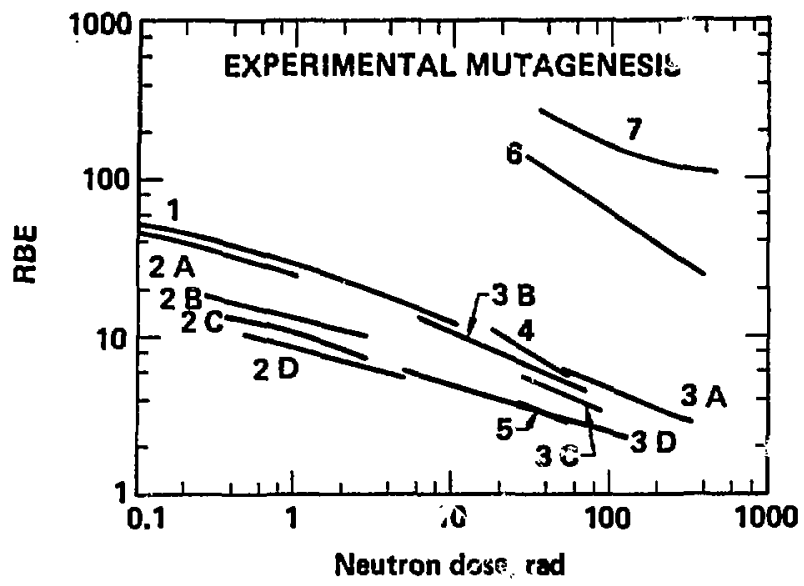

FIG. 2.6. Variation of neutron RBE with neutron dose for mutagenesis. Somatic mutations in Tradescantia stamen hairs with $0.43-\mathrm{MeV}$ neutrons (rurves $\mathrm{I}$ and $2 \mathrm{~A}$, with $\mathrm{T-MeV}$ neutrons (curve $2 \mathrm{~B}$ ), with 6-MeV neutrons (curve 2C), with 13.4-MeV neutrons (curve 2D). Chromosome aberrations in human 1 mphocytes with $0.7-\mathrm{MeV}$ neutrons (curve 3A), with 0.9-MeV neutrons (curve 3B), with $1.6-\mathrm{MeV}$ neutrons (curve $3 \mathrm{C}$ ), with $14.7 \mathrm{-MeV}$ neutrons (curve 30 ), and with $252 \mathrm{Cf}$ fission neutrons (curve 4). Spermatogonial translocations in the mouse with 0.7-Mel neutrons (curve 5). Somatic mutations in maize following irradiation of dry seeds with fission neutrons (curves 6 and 7). 
functions are rather consistent for all endpoints (witnin a factor of about two) in contrast to those observed for carcinogenesis (Fig. 2.4), and appear similar to RBE functions obtained for cell killing (Fig. 2.2). This would suggest that cell killing, usually cellular reproductive death (total impairment of the cell's ability for sustained division) is similar at the target level to mutagenesis; that is, that it involves DNA damage. However, tiie large differences in nautron RBE found between mutagenes is and some malignancies such as mouse ovarian tumors indicate that these particular malignancies are induced by different mechanisms than are point mutations or chromosome aberrations.

Neutron RBEs for mutation induction in dry plant seeds are exceptionally large (curves 6 and 7 in Fig. 2.6), probably as a result of the vell-known dependence of low-LET radiation damage on the water cuntent of material being irradiated $(22,23)$. Indirect radiation damage through radiolysis products formed in water are of considerable importance for lOW-LET radiation, whereas they are often of little significance for high-LET radiation such as neutrons. This resuits in exceptionally high RBEs when the water content of the irradiated material is low (because the low-LET comparison radiation is so ineffective). When these biological materials are soaked in water prior to irradiation, the neutron RBEs become similar to those for wet tissues $(22,23)$.

Somatic mutations ir Tradescantia stamen hairs (curves 1. and 2A-D) have been extensively studied e.g., $(4,5)$, and have been used in a major way to support the Theory of Dual Radiation Action (2). 
These experimenis are now considered here, and it will be pointed out that their support of this Theory is not without question.

The principal advantage of using the Tradescantia endpoint is the associated ability to detect significant effects at very low doses (down to about 0.01 rad of neutrons). The theory of Dual Radiation Action states in part that radiation absorption events in biological materials result in sublesions that must interact in order for radiation damage to appear. For high-LET radiation, e.g., 0.43-MeV neutrons, the proton recoil tracks are spar'sely spaced for dose levels of must interest here, hence lesions are formed almost entirely from interactions within tracks (rather than between tracks) and therefore yield effects that are proportional to the first power of the dose. However, low-LET radiation, e.g. garma rays, deposits energy much more uniformiy and therefore two different gamma-ray photons have a good chance (even at doses of only a few rad) of creating sublesions sufficiently close in both time and space to allow interaction between them. Therefore, the number of these interactions (and level of effect) depend, at least in part, upon the square of the dose. At very low doses (or low dose rates), the Theory predicts that low-LET radiation will also produce effects that are linear with dose, since sublesions from two independent events are unlikely to be near enough to each other (in either time or space) to interact. The Iradescantia data for pink mutations are shown in Fig. 2.7 for $0.43-\mathrm{MeV}$ neutrons and 250-kVp $\mathrm{x}$ rays and illustrate the points discussed above. A slope of +1 on the log-log plot indicates a first order linear function, white a slope of +2 indicates a second order quadratic expression. Interestingly, the 


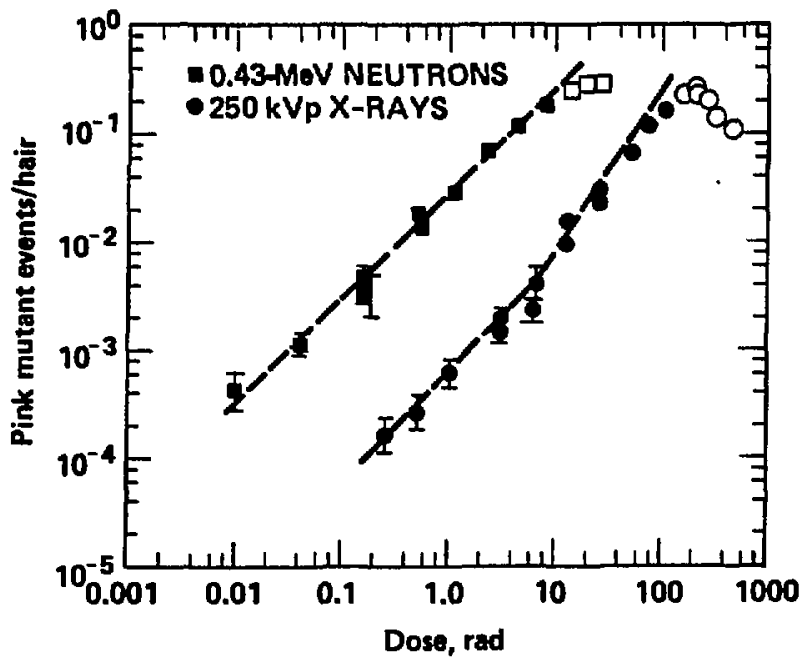

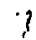

FIG. 2.7. Dose-response curves for somatic mutations in Tradescantia stamen hairs. The open data points at high doses (whose curve bend over, presumably from cell killing effets, is seen) were not used in curve fitting. This figure is modified from (4). See text for details. 
maximum slope observed for $250-k V p \times$ rays (at high doses) is about +7.4 (Fig. 2.7), which is less than the theoretical maximum value of +2 predicted by the Theory of Dual Radiation Action. This departule from the Theory may result from dose rate effects (i.e. . less than optimum dose rate) or from significant cell killing prior to the maximum slope being realized. It is also possible that the Theory of Dual Radiation Action simply does not adequately describe the effects observed in this endpoint and that more complex factors may be involved in the radiation effect process.

Neutron RBEs for heritable mutations in mouse spermatogonia are listed in Table 2.5 for fission neutrons. High values of RBE are attained for mutagenesis, which compare well with those from ciher biological materials (see Fig. 2.6). Mutations induced in mouse oocytes, however, show a more complicated relationship and are strongly influenced by the developmental stage of the oocyte at the time of irradiation, as seen in Table 2.6. The maturation period of mouse oocytes appears to be 6 to 7 weeks from stage $3 \mathrm{~b}$ of 0akberg to ovulation (30), and therefore irradiation within 7 weeks of conception insures that offspring are derived from oocytes that were mature or maturing at the time of exposure. These cells, not being especially sensitive to radiation killing, can survive exposure to several hundred rad of gamma rays and transmit mutations to the offspring $(30,39)$. The fission neutron dose-response for mutation induction in mature or maturing mouse oocytes are plotted in Fig. 2.8. RBEs are calculated from the linear regression of these data points and the following low-LET dose-response function: 
TABLE 2.5. RBE values for induction of specific locus and dominant mutations in mouse spermatogonia by fission neutrons.

\begin{tabular}{lllll}
\hline $\begin{array}{l}\text { Type of } \\
\text { mutation }\end{array}$ & $\begin{array}{l}\text { Dose rate } \\
\text { (rad/min) }\end{array}$ & $\begin{array}{l}\text { Low-LET } \\
\text { comparison }\end{array}$ & RBE & Reference \\
\hline Specific locus & $0.2-79$ & Acute $x$ & 5.8 & 36 \\
Specific locus & 0.1 & Chronic $\gamma$ & 18 & 36 \\
Specific locus & 0.002 & Chronic $\gamma$ & 23 & 37 \\
Specific locus & 0.001 & Chronic $\gamma$ & 17 & 38 \\
Dominant visible & $0.002-0.001$ & Chronic $\gamma$ & 19 & 37 \\
\hline
\end{tabular}

TABLE 2.6. Specific locus mutations after exposure of mouse oocytes to fission neutrons.

\begin{tabular}{lccc}
\hline $\begin{array}{l}\text { Interval between* } \\
\text { irradiation and } \\
\text { conception (weeks) }\end{array}$ & $\begin{array}{c}\text { Absorbed } \\
\text { dose } \\
(\mathrm{rad})\end{array}$ & $\begin{array}{c}\text { Frequency** } \\
\text { per locus per } \\
10^{5} \text { gametes }\end{array}$ & $\begin{array}{c}\text { Neutron } \\
\text { RBE }\end{array}$ \\
\hline & & & \\
$<7$ & 30 & 2.43 & 6.3 \\
& 60 & 12.29 & 4.5 \\
& 120 & 16.51 & 3.2 \\
& & & - \\
& 30 & 0.73 & - \\
& 60 & 0.00 & - \\
\hline
\end{tabular}

* See text for explanation.

** nata from reference 30 . 


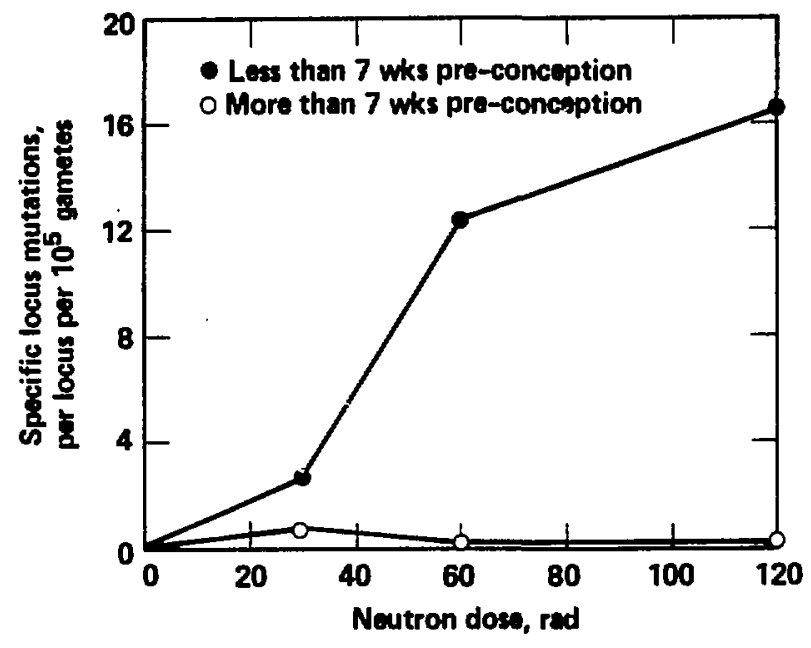

FIG. 2.8. Mutations induced by fission neutrons in mouse oocytes. See text for explanation. Plotted from tabulated data in (30). 


$$
\left.E=2.81 \times 10^{-8} D+1.15 \times 10^{-9} D^{2} \text { (from Reference } 30\right),
$$

where $E$ equals the specific locus mutation frequency per locus per $10^{5}$ gametes. The resulting RBEs (for intervals less than 7 weeks) are listed in the upper portion of Table 2.6 and are similar to those for other mutagenesis endpoints (see Fig. 2.6).

However, radiation exposure prior to seven weeks before conception insures that the offspring are products of oocytes that were immature at time of irradiation. Although the high radiosensitivity of these oocytes to killing results in almost total cell depletion following gamma-ray doses of 30 to 40 rads (40-42), the few offspring that are produced show no heritable mutations $(43,44)$. This is indeed an extraordinary departure from observations in mature mouse oocytes and spermatogonia. Explanations have been given for this peculiar lack of mutations. Russell (44) argues that highly efficient repair mechanisms in these cells virtually eliminate radiation-induced DNA lesions. Others conclude that repair mechanisms cannot possibly be that efficient, especially for neutron-induced damage, and therefore favor an alternate hypothesis that selection is operating to elininate cells carrying mutations. Neither of these explanations is totally convincing. An explanation that uses new data on oocyte killing (obtained here and presented in Chapters 3 and 4) will be given in Chapter 6 . 


\section{CATARACTOGENESIS}

Cataract formation in the mouse is a highly neutron-sensitive biological endpoint, allowing the demonstration of effects at very low doses $(7,8)$. The most widely quoted study is probably that by Bateman et al. (7) which demonstrated effects at $20 \mathrm{mrad}$ of neutrons, and RBEs approaching 200 (see Table 2.7). Results from a more recent. study (8) is also shown in Table 2.7. Neutron RBE-vs-dose curves from both studies are shown in Fig. 2.9 for each neutron energy used. Again RBEs are observed to increase with decreasing dose. However, values appear to be somewhat highe:" for cataractogenes is than for either cell killing or mutagenesis (compare Fig. 2.9 with Figs. 2.2 and 2.6). The reason for this is not understood; but gamma rays are known to be highly inefficient in producing cataracts (3).

\section{LIFE SHORTENING}

The shortening of lifespan in experimental animals following low to moderate radiation doses is essentially accounted for by induced malignancies (30). The neutron RBE for life shortening should therefore simply reflect the collective RBE values for the induced malignancies that would result in death; it should be equal to the weighted average of these values. For example, in RFM female mice the malignancies primarily induced are of reticular origin (1ymphomas and leukemias) (45). When the neutron RBE for life shortening in 
TABLE 2.7. Representative neutron RBE studies on experimental cataractogenes is.

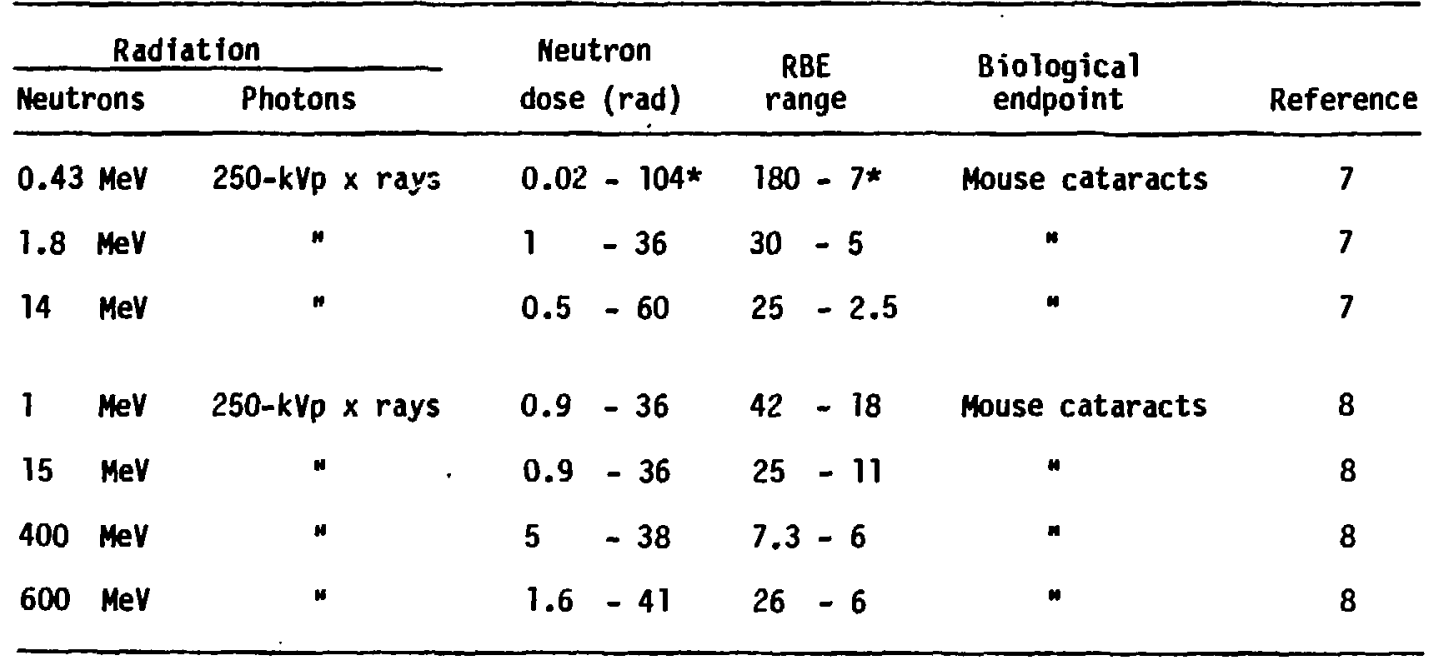

* See Table 2.2 footnote 


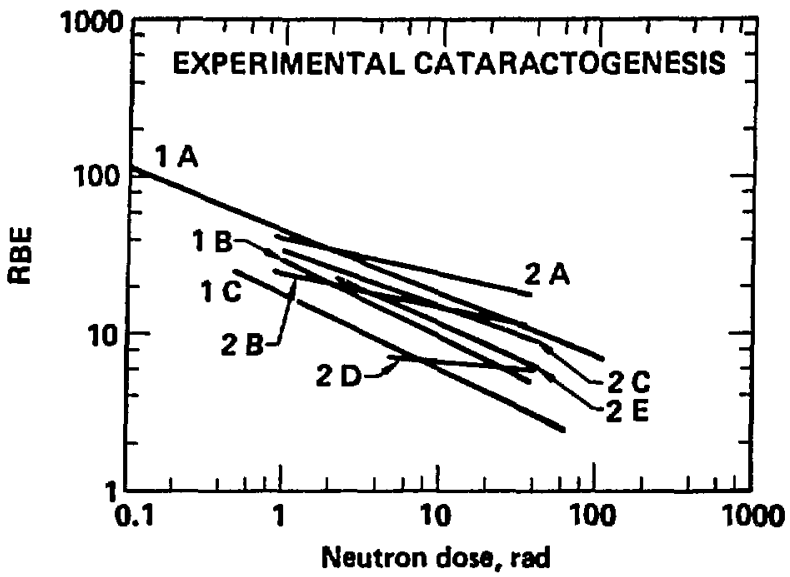

FIG. 2.9. Variation of neutron RBE with neutron dose for radiation-induced cataracts in mice. Lens opacities in albino Swiss females with $0.43-\mathrm{MeV}$ neutrons (curve $1 A$ ), with $1.8-\mathrm{MeV}$ neutrons (curve $(B)$, with $14-\mathrm{MeV}$ neutrons (curve $(C)$ ). Lens opacities in $\mathrm{F}$ ? hybrid between $\mathrm{C} 3 \mathrm{H} \times \mathrm{C57BL}$ mice with 1-MeV neutrons (curve $2 \mathrm{~A}$ ), with 5-MeV neutrons (curve 2B), with 15-MeV neutrons (curve $2 C$ ), with $400-\mathrm{MeV}$ neutrons (curve 2D), and with 600-MeV neutrons (curve $2 E$ ). See text for discussion. 
these animals (45) is plotted as a function of neutron dose (Fig. 2.10), together with neutron RBEs for thymic lymphoma and leukemia (26), we see, as expected, good agreement.

\section{EFFECT OF NEUTRON ENERGY ON RBE}

Several investigators have studied the effect of neutron energy on $\operatorname{RBE}(5,7,8,12-i 7,21,46,47)$, with the general conclusion that maximum effectiveness is obtained with neutrons of about $0.4 \mathrm{MeV}$. Lower RBEs are found with neutron energies above and below this value. However, since neutron dose also affects RBE, the proper way to determine the influence of neutron energy is to hold dose constant while varying only the energy. This has generally not been done, i.e., the level of effect has usually been kept constant while both neutron dose and energy are varied, which can result in misleading information. For example, neutron RBE for pink mutant events in Tradescantia stamen hairs (5) changes by a factor of more than $200 \%$ between 0.1 and $0.43 \mathrm{MeV}$ when level of effect is kept constant, whereas only $30 \%$ change is observed between these energies when the dose is kept constant, as shown in Table 2.8. Hence, at least some of the so called "neutron-energy-RBE effect" found in the literature is spurious, actually being due to effects of neutron dose rather than of meutron energy.

Figure 2.11 illustrates the true effect of neutron energy on RBE (neutron dose here is kept constant at $10 \mathrm{rad}$ ) for five endpoints. 


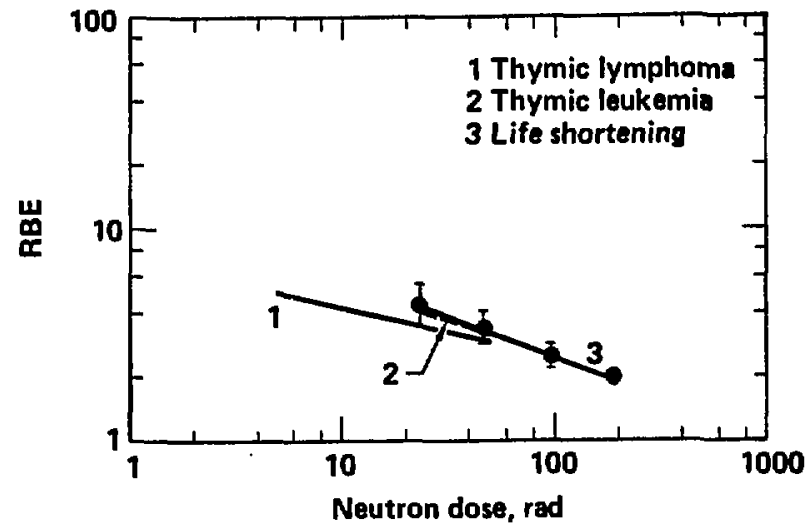

FIG. 2.10. Comparison of neutron RBE for life shurtening and the principal induced malignancies which result in death. See text for explanation. 
TABLE 2.8. Effect on neutron RBE of varying neutron energy.

\begin{tabular}{lcccc}
\hline $\begin{array}{l}\text { Neutron } \\
\text { energy } \\
\text { (MeV) }\end{array}$ & $\begin{array}{c}\text { RBE* at } \\
\text { constant } \\
\text { effect }\end{array}$ & $\begin{array}{c}\text { Neutron } \\
\text { dose } \\
\text { (rad) }\end{array}$ & $\begin{array}{l}\text { RBE* at } \\
\text { constant } \\
\text { neutron } \\
\text { dose }\end{array}$ & $\begin{array}{c}\text { Neutron } \\
\text { dose } \\
\text { (rad) }\end{array}$ \\
\hline 0.065 & 14.7 & 0.34 & 14 & 0.3 \\
0.110 & 21.7 & 0.23 & 20 & 0.3 \\
0.430 & 47.6 & 0.11 & 34 & 0.3 \\
1.020 & 18.5 & 0.27 & 18 & 0.3 \\
6.000 & 13.5 & 0.37 & 14 & 0.3 \\
13.400 & 10.4 & 0.48 & 12 & 0.3 \\
\hline
\end{tabular}

* For Tradescantia stamen-hair mutations.

Although RBEs are observed to decrease with increasing energy between about C. $.4 \mathrm{MeV}$ and $15 \mathrm{MeV}$, the magnitude of the decrease is considerably less than expected from analyses where the effect is kept constant $(5,7,8,10,11,19)$. Curves $A$ and $B$ are both for cataracts, but in two different mouse strains (given below), and show differences between them in RBEs of about two-fold. Since 250-kVp $x$ rays were used as the comparison radiation in both studies, it is unlikely that factors other than mouse-strain differences could result in such discrepancies (assuming dosinetry was adequate). The differences between curves $A$ and $B$ do not remain constant with neutron dose; that is, RBE does not vary equally with neutron dose for the two mouse strains (also compare curves $1 A$ and $2 A$ in Fig. 2.9). It appears that the RBE results obtained with FI offspring from C3HJ $\times$ C57BL mice (curves $2 A$ through $2 E$ in Fig. 2.9) 


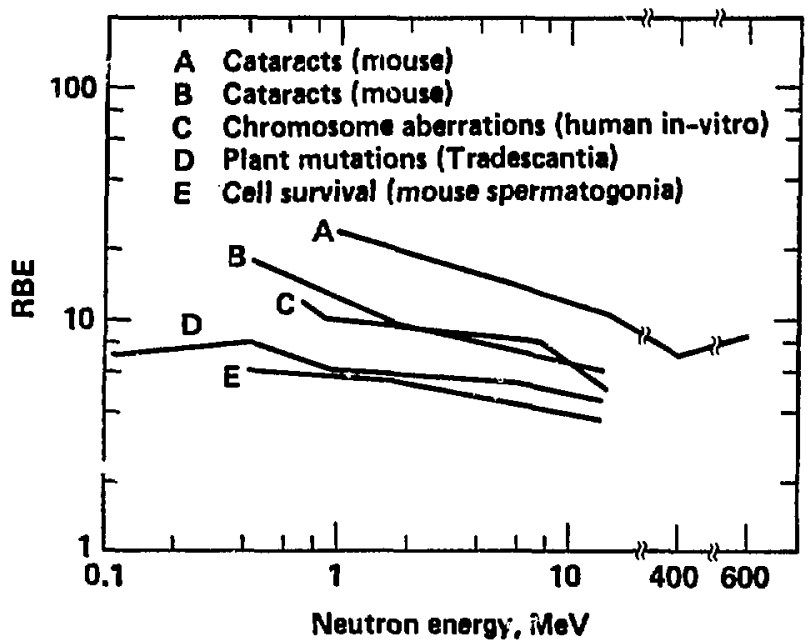

FIG. 2.11. Variation in neutron RBE with neutron energy. See text. 
yield lower slopes with neutron dose than do those for albino Swiss mice (curves $1 A$ through $I C$ in Fig. 2.9), indicating that the former may not reach the very high neutron RBEs ( 200 at $0.02 \mathrm{rad}$ ) previously obtained for cataract induction by Baternan et al. (7). Curves $C$ and $D$ in Fig. 2.11 are for in vitro human chromosome aberrations and Tradescantia somatic mutations respectively. They appear somewhat different from each other. However, the comparison radiation for curve $C$ was ${ }^{60}$ Co gamma rays whereas for curve $D$ it was 250-kVp $\times$ rays, and this could account for the observed difference (48). For type $B$ mouse spermatogonial survival data (curve $E$ in Fig. 2.11), ${ }^{60}$ Co gamma rays were used as the comparison radiation; yet these data yield the lowest neutron RBEs of all the endpnints shown in Fig. 2.11. This probably reflects their high gamma-ray sensitivity to cell killing.

Neutron RBEs generally decrease with increasing neutron energy between $0.4 \mathrm{MeV}$, and $15 \mathrm{MeV}$ and there is some (but more limited) evidence of decrease in the other direction, as energy decreases below $0.4 \mathrm{MeV}$. The influence of neutron exergy on RBE varies with endpoint, and among the effects shown in Fig. 2.11 appears greatei for cataractogenesis than for mutation induction and cell killing. 


\section{NEUTRON AND GAMMA-RAY EXPERIMENTS ON MOUSE OOCYTES}

Neutron RBE values for numerous endpoints have been reported and shown to vary with several physical and biological parameters (see Chapter 2). However, few meutron RBE studies have used biological endpoints capable of providing meaningful data at doses as low as the 1-rad range. Studies in that category are listed in Table 3.1, with indications of the lowest doses used and the RBEs found. It is seen that RBEs at low doses can reach high values and are remarkably similar for different eridpoints (see Fig. 3.1). Since knowledge of neutron RBEs at very low doses is based primarily on these few studies, which may not be broadly representative, it is important to investigate other highly radiosensitive biological endpoints as well.

TABLE 3.1. Results from very-low-dose reutron RBE studies.

\begin{tabular}{lllll}
\hline & $\frac{\text { Minimum dose used }}{\text { X ray or }}$ & $\begin{array}{c}\text { RBE at } \\
\text { minimum } \\
\text { Eamase used } \\
\text { (rad) }\end{array}$ & $\begin{array}{c}\text { Neutron } \\
\text { (rad) }\end{array}$ & $\begin{array}{c}\text { RBE at } \\
\text { neutrons }\end{array}$ \\
\hline $\begin{array}{l}\text { Tradescantia } \\
\text { mutations }\end{array}$ & 0.6 & 0.010 & 60 & 12 \\
$\begin{array}{l}\text { Mouse cataracts } \\
\begin{array}{l}\text { Rat mammary } \\
\text { tumors }\end{array}\end{array}$ & 4.5 & 0.022 & 204 & 15 \\
\hline
\end{tabular}




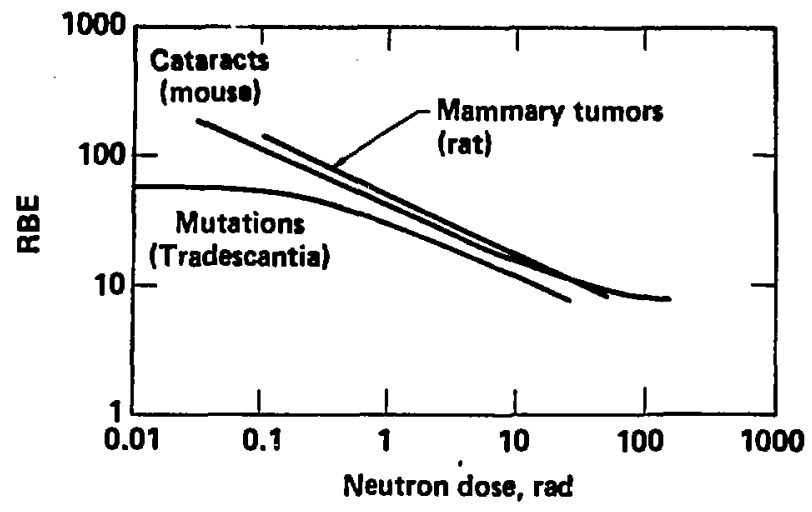

FIG. 3.1. Variation in neutron RBE with neutron dose for highty radiosens:tive biological endpoints. Redrawn from (1). 
The killing of immature oocytes (stages 1 and 2 of 0akberg, see Reference 51 ) in juvenile mice, which is among the most radiosensitive mamalian endpoints known, was chosen here. This endpoint shows a rather dramatic radiosensitivity relationship to mouse age (see Fig. 3.2). A very large increase in oocyte sensitivity occurs at or shortly after birth and this high level of sensitivity continues until about day 18 when it becomes smaller. The experiments described below provide neutron RBE values for mouse oocyte killing and explore how these RBEs are influenced by neutron energy, radiation dose, and the animal's age at exposure. An effort is made to identify the radiosensitive target* for mouse oocyte killing and to discuss the possible mechanism of radiation action.

MATERIALS AND METHODS

Experimental Animals

The animals used were non-inbred Swiss-Webster mice (obtained from Simonsen Laboratories, Gilroy, CA). They have been used extensively here at Lawrence Livermore National Laboratory (LLNL) for tritium toxicity and tritium RBE studies $(40,41,42)$. Mice were housed six to a cage, fed Purina mouse food and tap water ad-libitum, and cared for according to the standard procedures of the L L $M L$ animal

* The "target" refers here to the one (or more) subcellular site that must be sufficiently damaged by the radiation to $k i l l$ the cell. 


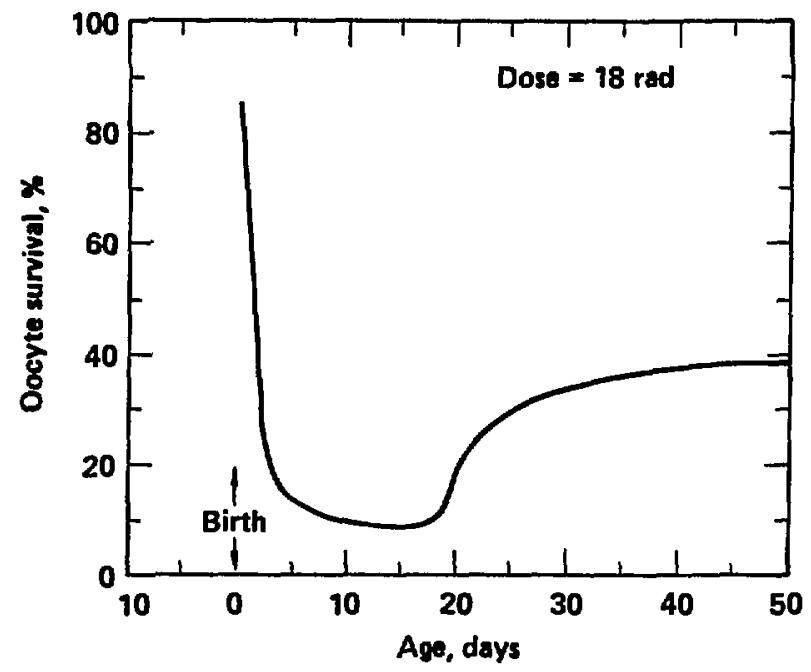

FIG. 3.2. Radiosensitivity of mouse oocytes as a function of the animal's age at exposure. 60 Co ganma rays were given at $1 \mathrm{rad} / \mathrm{min}$. Modified from (42). 
facility. The mice used in $0.43-\mathrm{MeV}$ neutron experiments, performed at Brookhaven National Laboratory, were shipped by air-freight from Simonsen's in California to BNL on Long Island where they were housed and cared for in animal facilities there.

\section{Cobalt-60 Gamma-Ray Exposures}

Two different ${ }^{60}$ Co gamma-ray sources were used, a $400-\mathrm{C} i$ source at Lawrence Berkeley Laboratory and a 6-Ci source at the LLNL Calibration and Standards Facility. Dosimetry was performed with both Victoreen ion chambers aud thermoluminescent dosimeters (TLDs). In one case (involving 100-rad/min exposures), TLDs were placed inside the peritoneal cavity (adjacent to the ovaries) in sacrificed 14-day-old mice. Routinely during radiation procedures, mice were contained in moderately snug perforated plastic capsules.

\subsection{3-MeV Neutron Exposures}

The 0.43-MeV neutron exposures were undertaken at the Brookhaven National Laboratory's Radiological Research Accelerator Facility (RARAF). The 3-MeV Van de Graaff machine produces almost monoenergetic neutrons at selected energias (in this case $0.43 \mathrm{MeV}$ ) by proton bombardment of a tritium target. Fourteen-day-old mice, and 11-day and 17-day post conception fetal mice (previously transported to Brookhaven from Simonsen's Laboratories in California) were placed in perforated plastic capsules and attached to a specially designed "Ferris-Wheel" rotating at 4 rpon about the tritium 
target (shown in Fig. 3.3). The angle from the target to the center of a mouse with respect to the proton beam axis was $114^{\circ}$. The mouse ovaries were at $22 \mathrm{~cm}$ from the target (approximating a point source at the distance), resulting in an absorbed dose rate to the ovaries of about $10 \mathrm{rad} / \mathrm{hr}$. Dosimetry was performed with a tissue-equivalent ion chamber and leucite phantoms simulating the mice. The ion chamber was located inside a phantom while the wheel was rotating. Dosimetry results are plotted in Fig. 3.4 for the dose transmission factor (absorbed dose in phantom per unit tissue kerma in air) as a function of leucite thickness (phantom radius). The dose transmission in the phantom is approximated by: OT $=1.0-0.0215 \mathrm{r}$, where $r$ is the phantom radius. This function is linear with depth and yields a $10 \mathrm{x}$ reduction in dose through $0.5 \mathrm{~cm}$ of tissue-equivalent material, the approximate tissue penetration distance to ovaries in 14-day-old mice. Penetration distances for 11-day and 17-day post conception fetal mice are estimated as $1.5 \mathrm{~cm}$ and $2.0 \mathrm{~cm}$ respectively, resulting in $32 \%$ dose reduction for 11 -day and $43 \%$ for 17 -day post conception animals. Figure 3.5 illustrates the effect on absorbed dose of lateral variations in mouse position. The maximum variation allowed by experimental design is estimated as $\pm 1 \mathrm{~cm}$, resulting in a dose uncertainty of not more than $\pm 5 \%$.

\section{Californium-252 Fission-Neutron Exposures}

${ }^{252} \mathrm{Cf}$ exposures were done at the LLNL Calitration and Standards Facility, using a source traceable to the National Bureau 


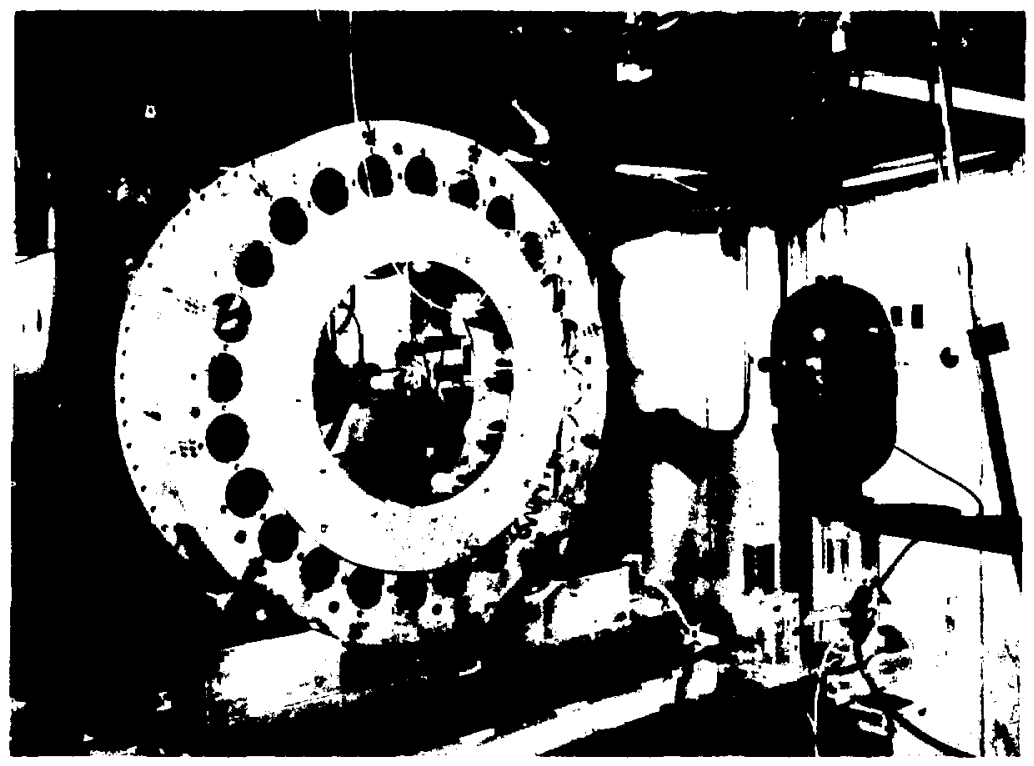

FIG. 3.3. Experimental set-up used for 0.43-MeV neutron exposures at RARAF. The mice were contained in the perforated plastic capsules (partially observed inside "Ferris-Wheel") while the wheel rotated around the accelerator ${ }^{3} \mathrm{H}$ target (seen at center of "Ferris-Whee 7") at $4 \mathrm{rpm}$. 


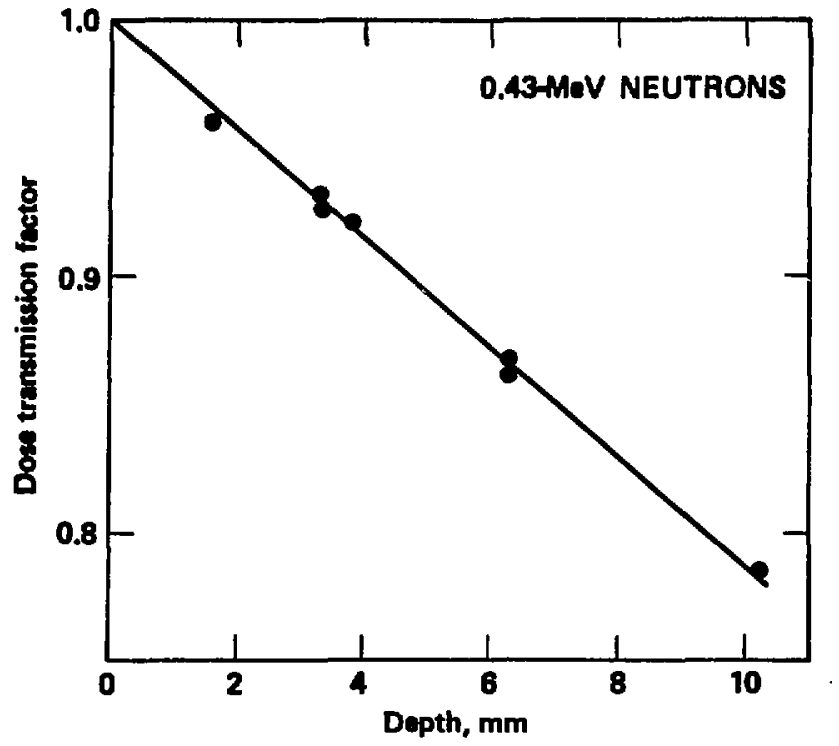

FIG. 3.4. Dose transmission of $0.43-\mathrm{MeV}$ neutrons through leucite phantorns of various thickness. Depth of penetration is equivalent to the phanthon radius. 


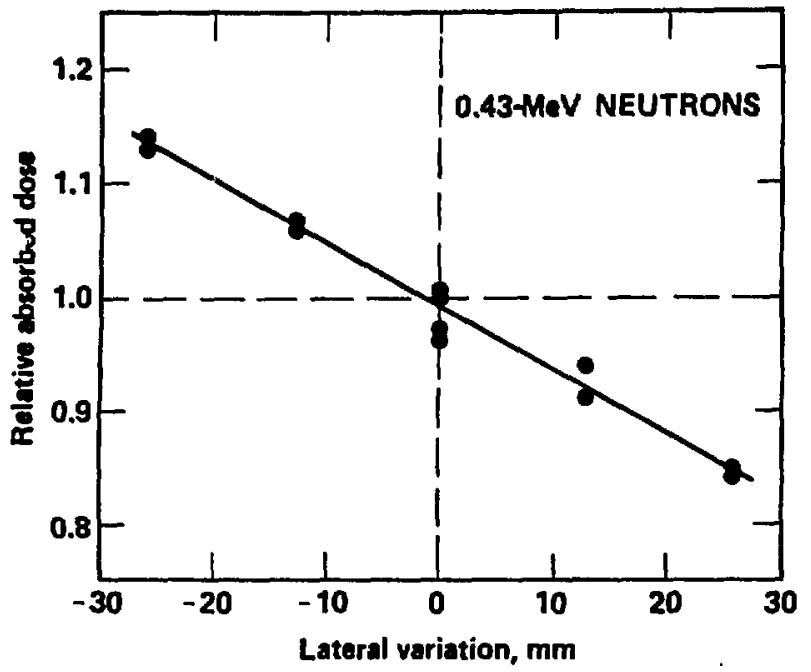

FI6. 3.5. The influence of lateral variations in mouse positioning on absorbed dose from $0.43-\mathrm{MeV}$ neutrons. 
of Standards. Figure 3.6 shows an uncollided ${ }^{252} \mathrm{Cf}$ neutron spectrum (49) which has peak intensity at about $0.8 \mathrm{MeV}$ and an average neutron energy of about $2.15 \mathrm{MeV}$. The ${ }^{252} \mathrm{Cf}$ fission neutrons used in this experiment were slightly degraded in energy from neutron interactions within the source and with intervening light structural materials, and were emitted with an average energy of about $1.8 \mathrm{MeV}$. In addition to the extensive dosimetric history of this source, tissue-equivalent ion chamber and Geiger-Muller dosimetric measurements were made. Table 3.2 lists dose rates for both neutrons and garma rays. Dose transmission through $0.5 \mathrm{~cm}$ of tissue is approximately 100\% for these neutron and gamma-ray energies. Perforated plastic capsules (containing the mice) were placed in an $A 1$ holder that was rotated about the ${ }^{252} \mathrm{Cf}$ source at $4 \mathrm{rpm}$. Mouse ovaries were at a distance of $10 \pm 0.5 \mathrm{~cm}$ from the source. Two small pflot experiments were also performed using dose rates different from those 1:sted in Tatle 3.2. In these experiments mice were kept in thin $A 1$ cages $(6 \mathrm{~cm}$ wide) at a distance of $80 \mathrm{~cm}$ or $100 \mathrm{~cm}$ from the ${ }^{252} \mathrm{Cf}$ source for two to three days. Dose rates were a few rad/day (see Table 3.8).

\section{5-MeV Neutron Exposures}

Neutrons of $15 \mathrm{Mel}$ were obtained from the Insulated Core Transformer (ICT) deuterium-tritium neutron generator at LLNL. Deuterium ions are accelerated into a rotating tritium target, producing an intense neutron source. The ICT has an output of up to about $4 \times 10^{12}$ neutrons per second in the energy range of $15 \mathrm{MeV}$. 


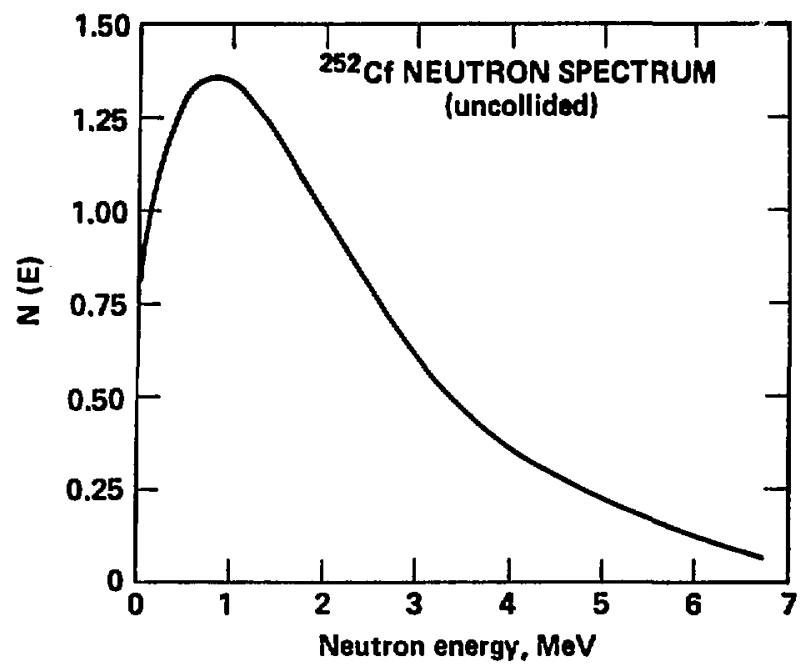

FIG; 3.6. Neutron energy spectrum from a very thin $252 \mathrm{Cf}$ source. $N(E)$ is the relative number of neutrons with energy (E). Drawn from data given in (49). 
TABLE 3.2. Neutron and garma-ray dose contributions from ${ }^{252} \mathrm{Cf}$ source.

Radiation

Dose rate*

$(\mathrm{rad} / \mathrm{hr})$

\begin{tabular}{lr} 
Neutrons & 8.5 \\
Gamma rays & 5.0 \\
Total & 13.5 \\
\hline
\end{tabular}

* At center of mouse.

Dosimetric characteristics of this facility are well known and have been published elsewhere (50). Mice were contained in perforated plastic capsules and placed in an Al fixture positioned $30 \mathrm{~cm}$ and $45 \mathrm{~cm}$ from the tritium target.

Preparation of Ovaries for Analysis

Mice were painlessly killed by cervical dislocation; ovaries were removed and allowed to fix in Helly's solution for 24 hours, washed in running tap water overnight, and placed into $75 \%$ ethyl alcohol. The remaining water content of the ovaries was removed by transferring them successively through higher alcohol concentrations, terminating with 100\%. The ovaries were then embedded in paraffin, serially sectioned at $5 \mu \mathrm{m}$, mounted as complete sets on slides, and stained with Delafield's hematoxyl in and eosin. 
Enumeration of 0ocytes

Immature oocytes in stages 1 and 2 of 0akberg (51) were counted at $1000 \mathrm{x}$, using a Leitz microscope fitted with an eyepiece grid. 0ocytes in every 20th section were counted. One ovary from each mouse was used and the other kept as a reserve.

\section{RESTiLiTS}

\subsection{3-MeV Neutron Exposure of Mice at 14 Days of Age}

The numbers of oocytes counted per ovary in control and exposed animals are given in Table 3.3, with the number of inice per dose group and oocyte survival (as percentage of control). Mice were killed at 35 days of age. In Fig. 3.7 oocyte survival is shown as a function of neutron tose. The data fit well the following exponential function

$$
S(x)=100 \mathrm{e}^{-0.1820},
$$

and it may be noted that the $D_{37}$ (the dose that reduces the surviving fraction to $1 / \mathrm{e}$ ) is only $5.5 \mathrm{rad}$. Oocyte dose-response data (except results from the explorat 'experiments where mice were exposed on the 11 th or 17 th postconception day) were fitted by linear-quadratic functions. The fitted linear-quadratic curve for $0.43-\mathrm{MeV}$ neutron irradiation at 14 days of age is statistically not significantly different $(p<0.05)$ from the exponential function above, shown in Fig. 3.7. 
TABLE 3.3. Effect of 0.43-MeV neutron exposure of mice at 14 days of age.

\begin{tabular}{lccr}
\hline $\begin{array}{l}\text { Dose } \\
\text { (rad) }\end{array}$ & $\begin{array}{c}\text { Number } \\
\text { of mice* }\end{array}$ & $\begin{array}{c}\text { Immature oocy:es } \\
\text { counted } \\
\text { per ovary** }\end{array}$ & $\begin{array}{c}\text { Oocyte } \\
\text { survival** } \\
\%\end{array}$ \\
\hline 0 (contro1) & 9 & $298 \pm 21.4$ & $100 \pm 7.2$ \\
0.1 & 11 & $287 \pm 18.5$ & $96 \pm 6.2$ \\
1 & 1 & 237 & 80 \\
5 & 12 & $134 \pm 12.6$ & $45 \pm 4.2$ \\
10 & 8 & $48 \pm 5.6$ & $16 \pm 1.9$ \\
20 & 10 & $12.6 \pm 4.6$ & $4 \pm 7.5$ \\
30 & 11 & $0.91 \pm 0.4$ & $0.31 \pm 0.13$ \\
\hline
\end{tabular}

Twelve mice were exposed in each dose group. Some died as a result of transportation from Brookhaven to Livermore. Mice used in the 1 rad dose group were "runts" even at time of exposure. They begar dying a few days after exposure. Only one survived to 35 days, the age of sacrifice. st Mean \pm SEM.

0.43-MeV Neutron Exposure on 17th Day Postconception

Results from mice exposed two days prior to birth (17 days postconception) and killed at 35 days of age are shown in Table 3.4. Here only two doses were used. This was an exploratory axperinent designed to estimate the neutron sensitivity just prior to birth, birth being a time at which, for garma rays at least, mouse immature oocytes are most radioresistant (see Fig. 3.2). The results demonstrate trat neutrons are also considerably less effective at 17 days postconception; no significant oocyte killing was observed at 3.8 rads, while this dose kills about $50 \%$ of the oocytes in the 14-day-old mouse. Assuming an exponential survival curve with 


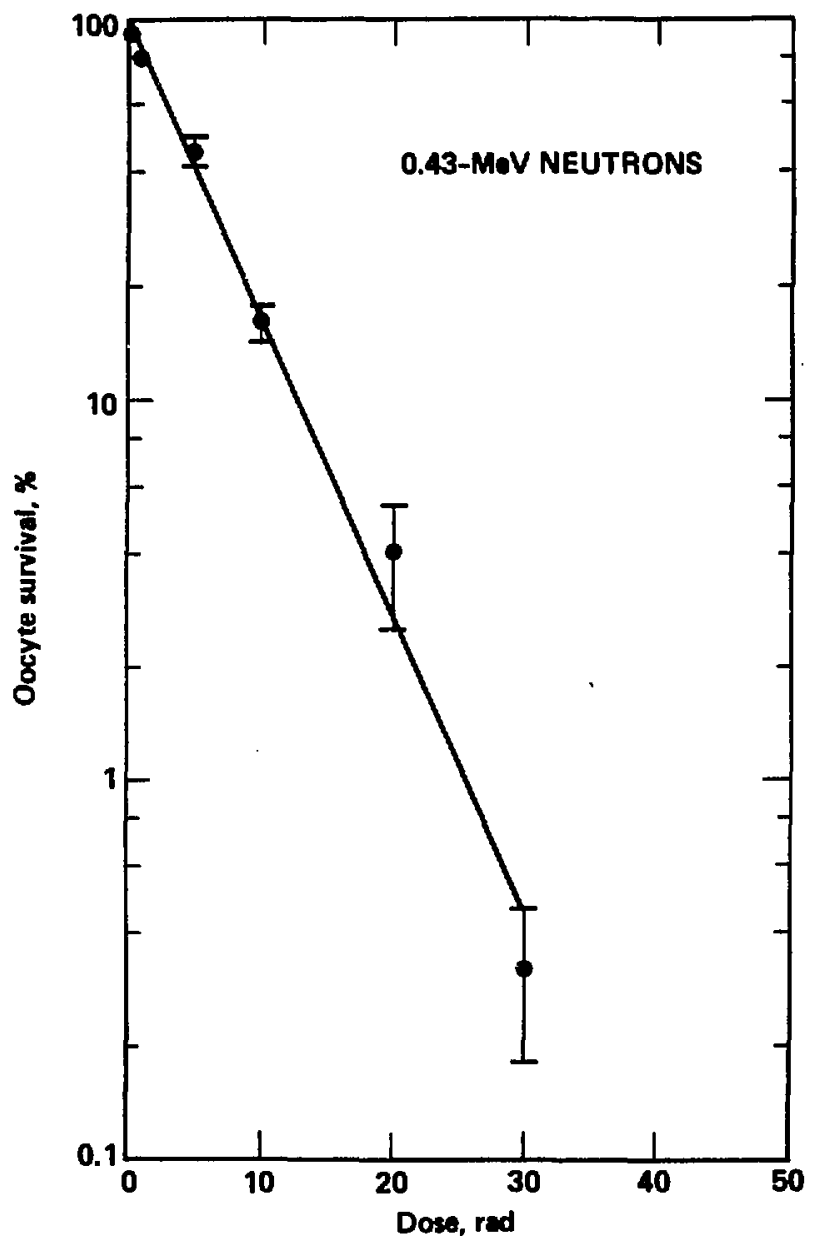

FIG. 3.7. Oocyte survival following $0.43-\mathrm{MeV}$ neutron irradiation of mice at 14 days of age. Error bars are \pm 1 SEM. 
$D_{37}$ of $17 \mathrm{rad}$, the following function fits the data in Table 3.4,

$$
S(x)=100 e^{-0.0460} \text {, }
$$

and in Fig. 3.8 these results are compared with oocyte survival functions for exposures at other ages.

TABLE 3.4. Effect of $0.43-\mathrm{MeV}$ neutron exposure on the 17 th day postconception.

\begin{tabular}{lccc}
\hline $\begin{array}{l}\text { Dose } \\
(\mathrm{rad})\end{array}$ & $\begin{array}{c}\text { Number } \\
\text { of mice* }\end{array}$ & $\begin{array}{c}\text { Immature oocytes } \\
\text { counted } \\
\text { per ovary** }\end{array}$ & $\begin{array}{c}\text { Oocyte } \\
\text { survival** } \\
(\mathrm{x})\end{array}$ \\
\hline 0 (control) & 10 & $264 \pm 32.9$ & $100 \pm 12.4$ \\
3.8 & 5 & $268 \pm 32.7$ & $101 \pm 12.4$ \\
30.4 & 7 & $46 \pm 11.7$ & $17 \pm 4.4$ \\
\hline
\end{tabular}

* The number of mice per dose group is influenced by litter size and the number of females per litter.

** Mean \pm SEM.

\subsection{3-MeV Neutron Exposure on 11th Day Postconception}

The effect of neutron exposure on the 11th postconception day (and mice killed at 35 days of age) is shown in Table 3.5. This particular time of exposure was chosen to coincide with the period of active oogonial mitotic division (which produces the oocyte supply). It should be possible then to induce reproductive death in the multiplying oogonia, and in this way obtain dose-response functions comparable to those for other rapidly dividing cells. Again assuming 


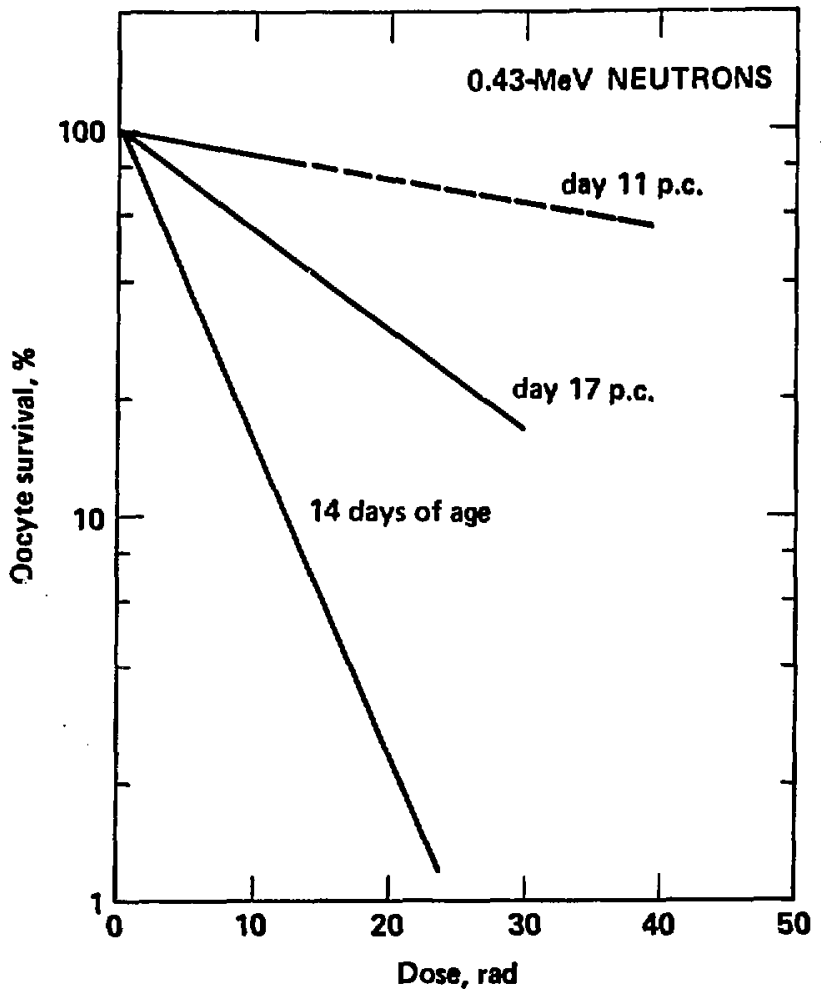

FIG. 3.8. A comparison of oocyte survival following $0.43-\mathrm{MeV}$ neutron irradiation on the 17 th or 17 th day post conception or at 14 days of age. 
the survival curve to be exponential, the following function was fit to the data given in Table 3.5,

$$
S(x)=100 e^{-0.01330}
$$

TABLE 3.5. Effect of $0.43-\mathrm{MeV}$ neutron exposure on the 11 th day postconception.

\begin{tabular}{lccc}
\hline $\begin{array}{l}\text { Dose } \\
\text { (rad) }\end{array}$ & $\begin{array}{c}\text { Number } \\
\text { of mice* }\end{array}$ & $\begin{array}{c}\text { Immature oocytes } \\
\text { counted } \\
\text { per ovary** }\end{array}$ & $\begin{array}{c}\text { oocyte } \\
\text { survival** } \\
(\boldsymbol{x})\end{array}$ \\
\hline 0 (control) & 10 & $358 \pm 20.9$ & $100 \pm 5.8$ \\
13.6 & 8 & $298 \pm 37.8$ & $83 \pm 10.6$
\end{tabular}

* The number of mice per dose group is influenced by litter size and the number of females per litter. $\star \star$ Mean \pm SEM.

The $\mathrm{O}_{37}$, determined by extrapolating the exponential survival curve (see Fig. 3.8), is 75 rad, considerably larger than that found for 17-day-postconception exposures. Indeed, it is comparable to values generally found for other reproducing cells.

${ }^{60}$ Co Gamma-Ray Exposures

Cobalt-60 gamma rays are used here as the reference radiation for RBE determination. Survival curves have previously been determined for mouse oocytes with ${ }^{60}$ Co gamma rays $(41)$, and these curves, with some additional experiments, are used here. The ${ }^{60} C_{0}$ dose rate of $1 \mathrm{rad} / \mathrm{min}$ used previously (41) differs from those used 
here for neutrons. It was necessary therefore to determine whether dose-rate effects with these neutron radiations could significantly influence RBE estimates. The approximately exponential survival curves for $0.43-\mathrm{MeV}$ and fission neutrons indicate that these radiations are not expected to be dose-rate dependent. Fifteen-MeV neutrons, however, yield a non-exponential survival curve, indicating that recovery may occur and making dose-rate considerations necessary in RBE analysis. Since $15-\mathrm{MeV}$ neutrons were given at higher dose rates $(25-50 \mathrm{rad} / \mathrm{min})$ than were ${ }^{60}$ Co gamma rays $(1 \mathrm{rad} / \mathrm{min})$, additional experiments were carried out to determine the possible influence of such dose-rate differences on oocyte killing. Table 3.6 presents data from ${ }^{60}$ Co dose-rate experiments showing that there are no significant differences in the percent survival of oocytes irradiated at $1 \mathrm{rad} / \mathrm{min}$ and at $100 \mathrm{rad} / \mathrm{min}$. Hence ${ }^{60} \mathrm{Co}$ gamma rays given at $1 \mathrm{rad} / \mathrm{min}$ can be used as the comparison radiation for $15-\mathrm{MeV}$ neutron RBE estimation.

${ }^{60}$ Co Gamma Rays and 0.43-MeV Neutrons Compared

Docyte survival curves for $0.43-\mathrm{MeV}$ neutrons and ${ }^{60} \mathrm{Co}$ gamma rays are compared in Fig. 3.9. Neutron RBEs are calculated from the curves by taking ratios of doses for equal effects. The RBE at any neutron dose can be obtained from the following derived function

$$
R B E=\frac{-a+\left(a^{2}+4 b c D_{n}\right)^{1 / 2}}{2 b D_{n}} \text {, }
$$


TABLE 3.6. Results for dose-rate e:periments with ${ }^{60}$ Co gama rays.

\begin{tabular}{cccccc}
\hline $\begin{array}{c}\text { Dose } \\
(\mathrm{rad})\end{array}$ & $\begin{array}{c}\text { Dose } \\
\text { rate } \\
\text { (rad/min) }\end{array}$ & $\begin{array}{c}\text { Number of } \\
\text { animals }\end{array}$ & $\begin{array}{c}\text { Age at } \\
\text { exposure } \\
\text { (days) }\end{array}$ & $\begin{array}{c}\text { Immature oocytes } \\
\text { ccunted } \\
\text { per ovary* }\end{array}$ & $\begin{array}{c}\text { Oocyte } \\
\text { survival* } \\
(\mathrm{x})\end{array}$ \\
\hline $0 * *$ & 0 & 6 & 18 & 324 & 100 \\
8.6 & 1 & -- & 18 & -- & $40+$ \\
8.6 & 100 & 6 & 18 & $144 \pm 22.7$ & $45 \pm \cdot 7$ \\
\hline
\end{tabular}

* Mean + SEM.

** Contrōls for $100 \mathrm{rad} / \mathrm{min}$ exposures.

+ This value was taken from the fitted survival curve since a data point did not exist for this particular dose (from Reference 41 ).

where $a$ and $b$ are linear and quadratic coefficients from the fitted ${ }^{60}$ Co gamma-ray dose-response curve and $c$ is the linear coefficient for $0.43-\mathrm{MeV}$ neutrons. Figure 3.10 shows RBE, determined with this formula, as a function of neutron dose. RBE increases in value with decreasing dose, reaching a constant maximum of 1.9 at very low doses.

\section{Fission Neutron Exposure}

The effects of ${ }^{252} \mathrm{Cf}$ fission irradiation are shown as a function of dose in Table 3.7 and Fig. 3.11. Doses were not pure neutrons; points with closed circles in Fig. 3.11 had about $37 \%$ gamma-ray contribution, the open circle had $27 \%$ and the open triangle about $43 \%$. Table 3.8 shows the effect of varying the dose rate (the data are from three experiments using different numbers of animals), and indicates that this parameter does not significantly influence the effectiveness of the ${ }^{252} \mathrm{Cf}$ radiation, at least for the doses 


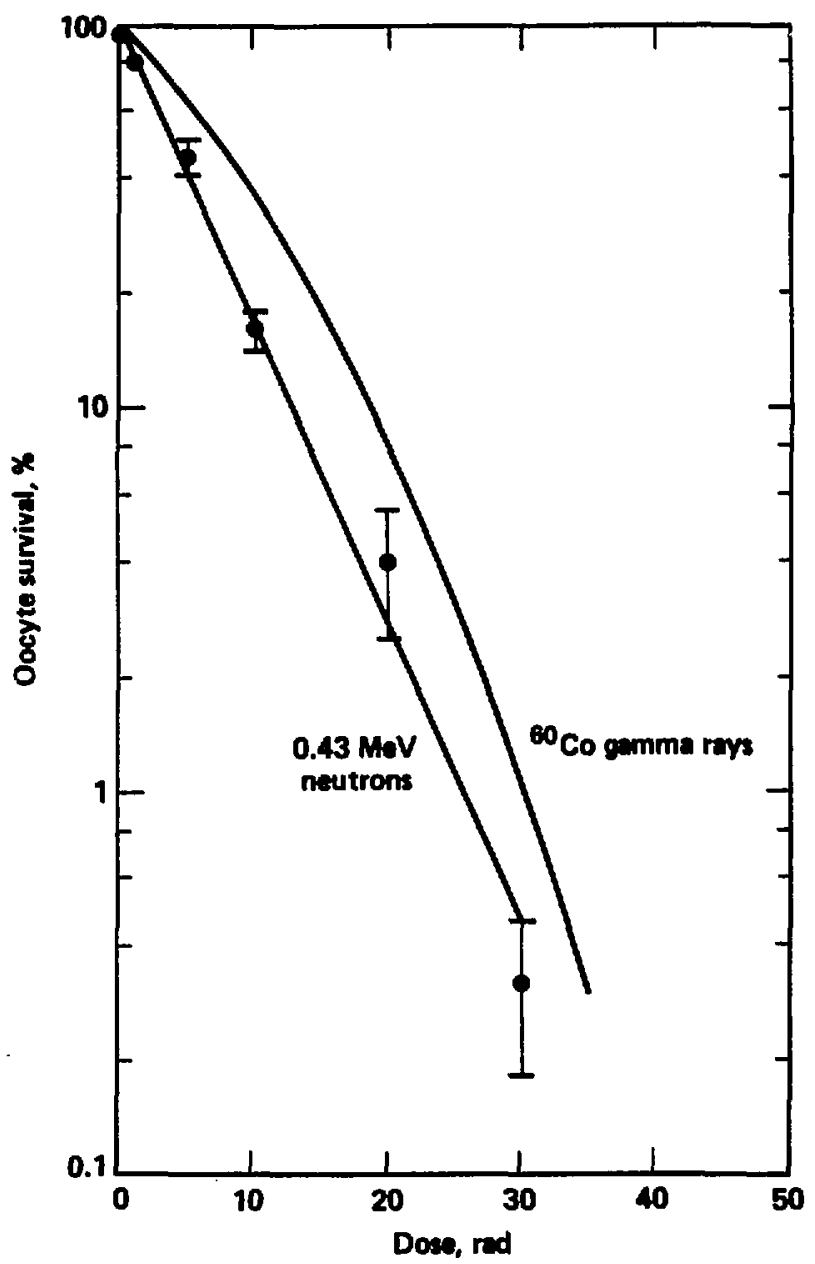

FIG. 3.9. A comparison of oocyte survival following irradiation with $0.43-\mathrm{MeV}$ neutrons and with $60 \mathrm{CO}$ gamma rays. Error bars on $0.43-\mathrm{MeV}$ neutron points are \pm 1 SEM. 


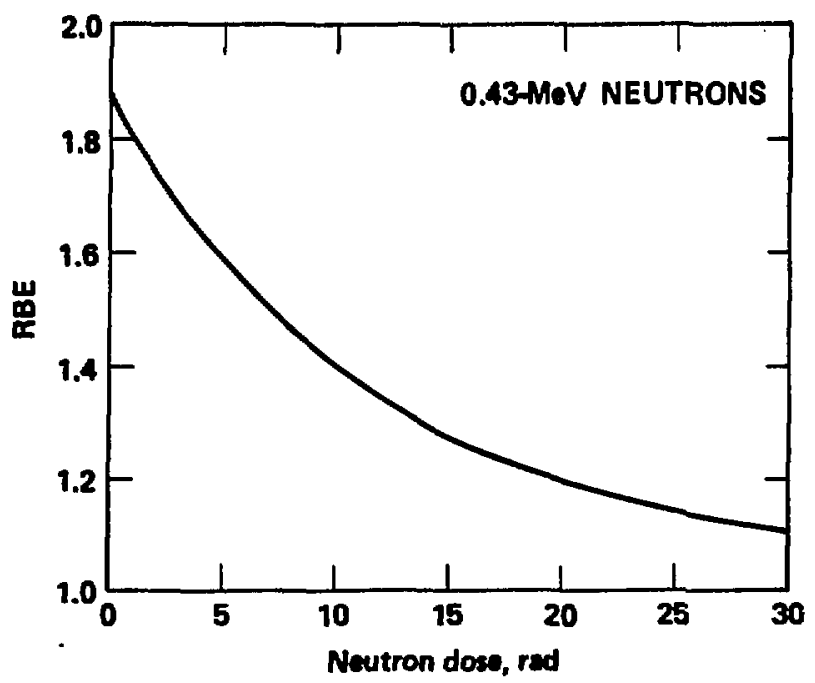

FIG. 3.10. RBE for oocyte killing with $0.43-\mathrm{MeV}$ neutrons as a function of neutron dose. See text. 
TABLE 3.7. Effect of Californium-252 exposure of 14-day-0ld mice.

\begin{tabular}{lccc}
\hline $\begin{array}{l}\text { Dose } \\
(\mathrm{rad})\end{array}$ & $\begin{array}{c}\text { Number } \\
\text { of mice }\end{array}$ & $\begin{array}{c}\text { Immature oocytes } \\
\text { counted } \\
\text { per ovary* }\end{array}$ & $\begin{array}{c}\text { Oocyte } \\
\text { survival* } \\
(\mathrm{x})\end{array}$ \\
\hline 0 (control) & 4 & $361 \pm 32.1$ & $100 \pm 8.9$ \\
10 & 4 & $178 \pm 19.6$ & $49 \pm 5.4$ \\
20 & 4 & $59 \pm 4.3$ & $17.3 \pm 1.2$ \\
30 & 4 & $24 \pm 1.6$ & $6.7 \pm 0.4$ \\
\hline
\end{tabular}

*Mean \pm SEM.

used here. The effect of pure fission neutrons can be estimated by subtracting the gamma-ray effect from the total. To do this, we assume that fission garma rays have the same effectiveness as ${ }^{60} \mathrm{Co}_{0}$ garma rays delivered at similar dose rates (this is reasonable since their average energies are similar). The derived function for oocyte survival following pure fission neutron exposure is:

$$
\begin{aligned}
& S_{n}=\exp -\left[\left(7.3 \times 10^{-2}\left(1.6 D_{n}\right)+5.6 \times 10^{-4}\left(1.6 D_{n}\right)^{2}\right.\right. \\
&-\left(6.0 \times 10^{-2}\left(0.6 D_{n}\right)+10^{-3}\left(0.6 D_{n}\right)^{2}\right. \\
&\left.-2\left(10^{-3} \times 510^{-4}\right)^{1 / 2} 0.6 D_{n}^{2}\right] .
\end{aligned}
$$

The first two terms in this function account for the effect from total ${ }^{252}$ Cf fission radiation (neutrons and gammas), the next two terms account for effects estimated from ${ }^{252} \mathrm{Cf}$ fission gamas alone, and the last term accounts for effects from interactions 


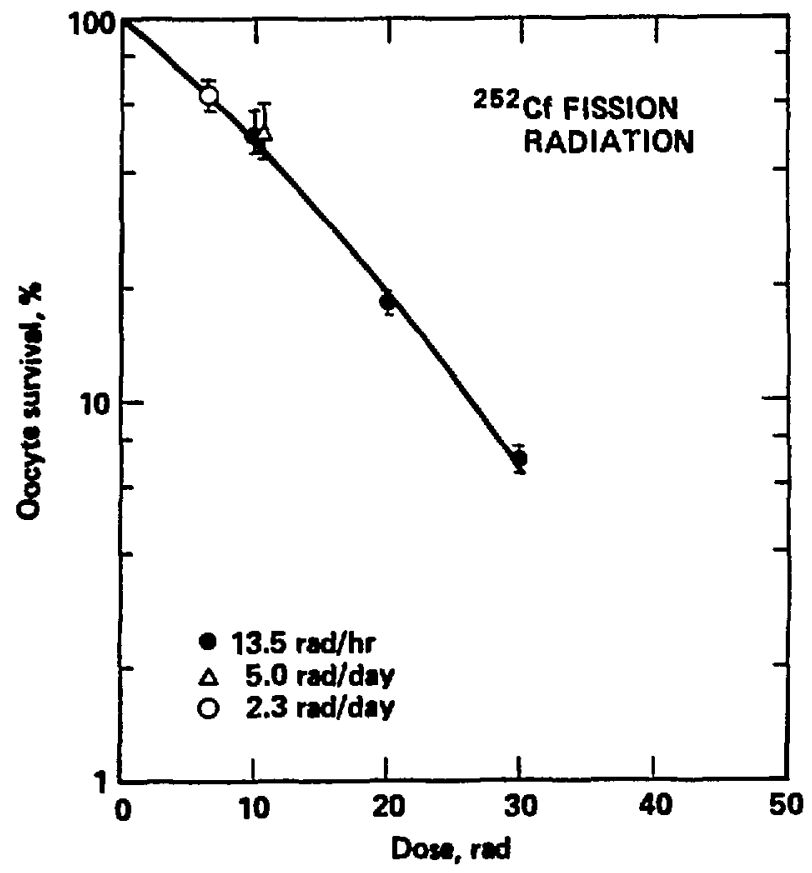

FIG. 3.11. Oocyte survival following ${ }^{252}$ Cf fission irradiation (including both neutrons and garma rays). Three dose rates were used. Error bars are \pm 1 SEM. 
TABLE 3.8. Effect of Californium-252 exposures at several dose rates.

\begin{tabular}{lccccc}
\hline $\begin{array}{l}\text { Dose-rate } \\
\text { (rad/day) }\end{array}$ & $\begin{array}{c}\text { Neutron } \\
\text { dose } \\
(\mathrm{rad})\end{array}$ & $\begin{array}{c}\text { Garma } \\
\text { dose } \\
(\mathrm{rad})\end{array}$ & $\begin{array}{c}\text { Number } \\
\text { of mice }\end{array}$ & $\begin{array}{c}\text { Immature oocytes } \\
\text { counted } \\
\text { per ovary* }\end{array}$ & $\begin{array}{c}\text { oocyte } \\
\text { surviva 1* } \\
(\boldsymbol{x})\end{array}$ \\
\hline control** & 0 & 0 & 3 & $433 \pm 38.9$ & $100 \pm 9.0$ \\
controlt & 0 & 0 & 10 & $340 \pm 27.9$ & $100 \pm 8.2$ \\
controltt & 0 & 0 & 4 & $361 \pm 32.1$ & $100 \pm 8.9$ \\
2.3 & 4.8 & 1.8 & 7 & $269 \pm 14.8$ & $62 \pm 5.5$ \\
5.0 & 6.1 & 4.6 & 5 & $166 \pm 13.1$ & $49 \pm 7.9$ \\
324 & 6.3 & 3.7 & 4 & $178 \pm 9.6$ & $49 \pm 5.4$ \\
\hline
\end{tabular}

* Mean + ISE.

** Contrōls for $2.3 \mathrm{rad} /$ day group.

+ Controls for $5.0 \mathrm{rad} /$ day group.

t+ Controls for $324 \mathrm{rad} /$ day group.

between neutron and garma-ray tracks (these interactions, or "synergisms" as they have sometimes been referred to, are discussed and formalized below). The percent survival calculated from this function is shown in Table 3.9 for the doses used in these experiments and the resulting oocyte survival curve (seen in Fig. 3.12 together with the curve for ${ }^{60}$ Co gamma rays) appears approximately exponential, indicating a nearly single-hit oocyte. killing mechanism, with $\mathrm{D}_{37}$ of 11.5 rad. Neutron RBEs calculated from these functions are shown in Fig. 3.13.

It has recently been reported that mixed gamma ray and neutron irradiation may result in "synergistic" effects $(53,54)$. A theoretical model based on the Theory of Dual Radiation Action (2) has been derived to estimate these effects (55) and is used here to 
TABLE 3.9. Estimated effect of pure fission neutron exposure.

\begin{tabular}{cc}
\hline $\begin{array}{c}\text { Neutron } \\
\text { dose } \\
(\mathrm{rad})\end{array}$ & $\begin{array}{c}\text { Oocyte } \\
\text { survival } \\
(\%)\end{array}$ \\
\hline 0 & 100 \\
10 & 43.6 \\
20 & 18.2 \\
30 & 7.2 \\
\hline
\end{tabular}

determine their influence on oocyte kflling by ${ }^{252} \mathrm{Cf}$ radiation. The function for percent survival is:

$$
\begin{aligned}
S(x)=100 \exp -\left[\left(a_{1} D_{1}\right.\right. & \left.+b_{1} D_{1}^{2}\right)+\left(a_{2} D_{2}+b_{2} D_{2}^{2}\right) \\
& \left.+2\left(b_{1} b_{2}\right)^{1 / 2} D_{1} D_{2}\right],
\end{aligned}
$$

where subscripts 1 and 2 denote coefficients and doses for radiations of type 1 (neutrons) and type 2 (ganma rays) respectively. The term describing "synergism" is

$$
F=2\left(b_{1} b_{2}\right)^{1 / 2} D_{1} D_{2}
$$

which accounts for inter-track interactions between neutrons and gamma rays. Table 3.10 shows tne expected effect on oocyte killing from such interactions. The quadratic coefficient for ${ }^{252} \mathrm{Cf}$ gamma rays $\left(b_{1}\right)$ was taken as intermediate between the quadratic coefficients for chronic and acute ${ }^{60}$ Co gamma irradiation. The 


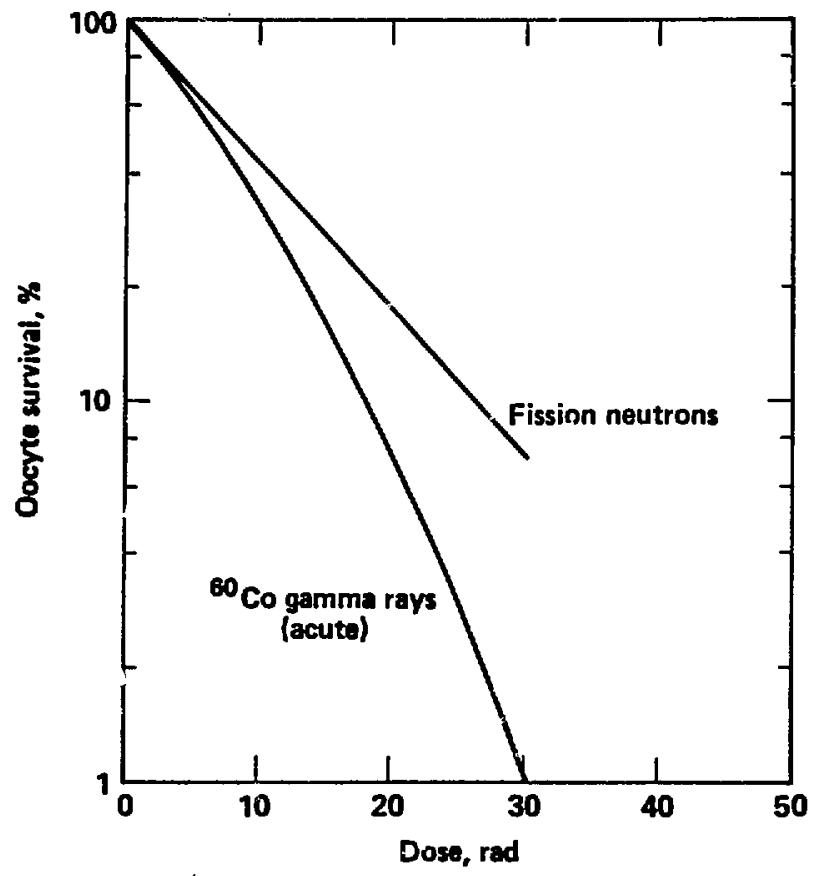

FI. 3.12. A comparison of oocyte survival following irradiation witn "pure" fission neutrons or ${ }^{60}$ Co gamma rays (see text). 


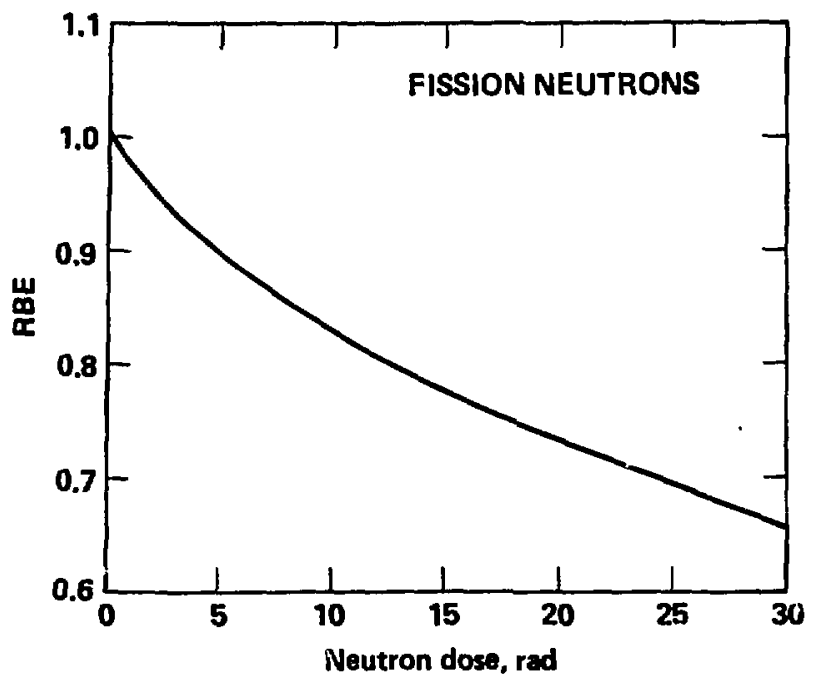

FIG. 3.13. RBE for fission neutrons as a function of neutron dose. 
TABLE 3.10. Effect of neutron and gamma-ray synergisms for ${ }^{252} \mathrm{Cf}$ exposures.

\begin{tabular}{cccccc}
$\begin{array}{c}\text { Neutron } \\
\text { dose } \\
\text { (rad) }\end{array}$ & $\begin{array}{c}\text { Gamma-ray } \\
\text { dose } \\
(\mathrm{rad})\end{array}$ & $\begin{array}{c}\mathrm{b}_{2}{ }^{*} \\
\left(\times 10^{4}\right)\end{array}$ & $\begin{array}{c}\mathrm{b}_{1}+ \\
\left(\times 10^{3}\right)\end{array}$ & $\mathrm{F}$ & $\begin{array}{c}\text { Oocyte } \\
\text { survival } \\
(x)\end{array}$ \\
\hline 10 & 5.9 & 2.4 & 1 & .058 & 94 \\
20 & 11.8 & 2.4 & 1 & .231 & 79 \\
30 & 17.7 & 2.4 & 1 & .520 & 59 \\
40 & 23.6 & 2.4 & 1 & .925 & 40 \\
\hline
\end{tabular}

* Quadratic coefficient for fission neutrons.

t Quadratic coefficient for gama-rays.

zoefficient $\left(b_{2}\right)$ for ${ }^{232} \mathrm{Cf}$ fission neutrons was obtained from a linear-quadratic, least squares best fit to the data in Table 3.9. The effects from ${ }^{252} \mathrm{Cf}$ fission radiation, ${ }^{252} \mathrm{Cf}$ fission neutrons, fission gamma rays, and neutron-garma interactions are compared in Fig. 3.14 and clearly show that oocyte killing from the interactions are, in relative terms, unimportant in this case.

Effect of 15-MeV Nes, is

Results from 15-MeV neutron irradiation given on the 18th day of age are given in Table 3.11 . The data were fitted by the least squares method to the 1 inear-quadratic function

$$
S(x)=100 \exp -\left[9.5 \times 10^{-2} D+1.7 \times 10^{-3} D^{2}\right],
$$




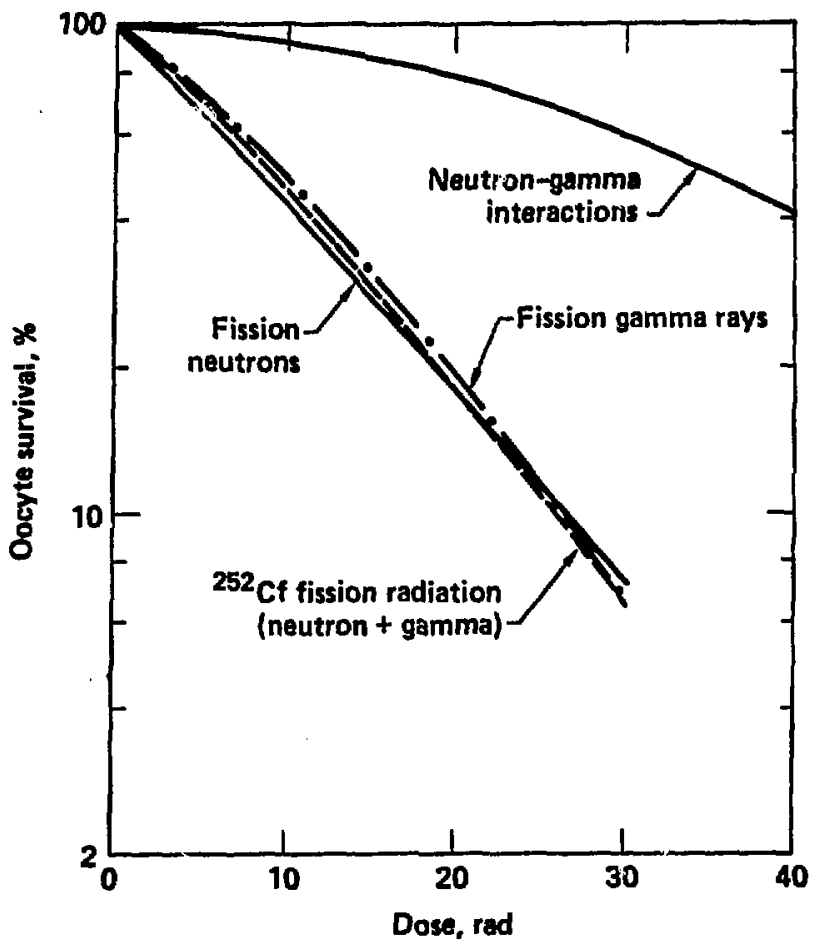

FIG. 3.14. Oocyte survival following irradiation with ${ }^{252} \mathrm{Cf}$, showing estimated effect of fission neutrons, fission gamma rays, and neutron-garma ray interactions. 
and the resulting survival curve is shown in Fig. 3.15. Slight curvature is indicated, which would reflect multiple hit oocyte killing. The $D_{37}$ is about 8 rad. In Fig. 3.16 the fitted survival curves for $15-\mathrm{MeV}$ neutrons and ${ }^{60} \mathrm{Co}$ gamma rays are compared. Interestingly, these curves are almost identical. That is, $15-\mathrm{MeV}$ neutrons are not any more effective than ${ }^{60}$ Co gamma rays. The RBE is therefore not different from unity at all doses tested. This is in great contrast to findings in many other experimental systems (see Chapter 2) where RBEs for 14 to $15-\mathrm{MeV}$ neutrons of more than 20 have been observed at low doses.

TABLE 3.17. 15-MeV neutron exposure at 18 days of age.

\begin{tabular}{lccr}
\hline $\begin{array}{l}\text { Dose } \\
\text { (rad) }\end{array}$ & $\begin{array}{c}\text { Number } \\
\text { of mice }\end{array}$ & $\begin{array}{c}\text { Immature oocytes } \\
\text { counted per } \\
\text { ovary }\end{array}$ & $\begin{array}{c}\text { oocyte* } \\
\text { survival } \\
(\chi)\end{array}$ \\
\hline 0 (control) & 20 & $369 \pm 27.7$ & $100 \pm 7.5$ \\
4.5 & 11 & $231 \pm 24.0$ & $62 \pm 6.5$ \\
9 & 10 & $156 \pm 11.8$ & $42 \pm 3.2$ \\
12 & 8 & $73 \pm 7.0$ & $20 \pm 1.9$ \\
24 & 8 & $17 \pm 5.5$ & $4.6 \pm 1.5$ \\
\hline *Mean \pm SEM. & & $\vdots 1$ &
\end{tabular}




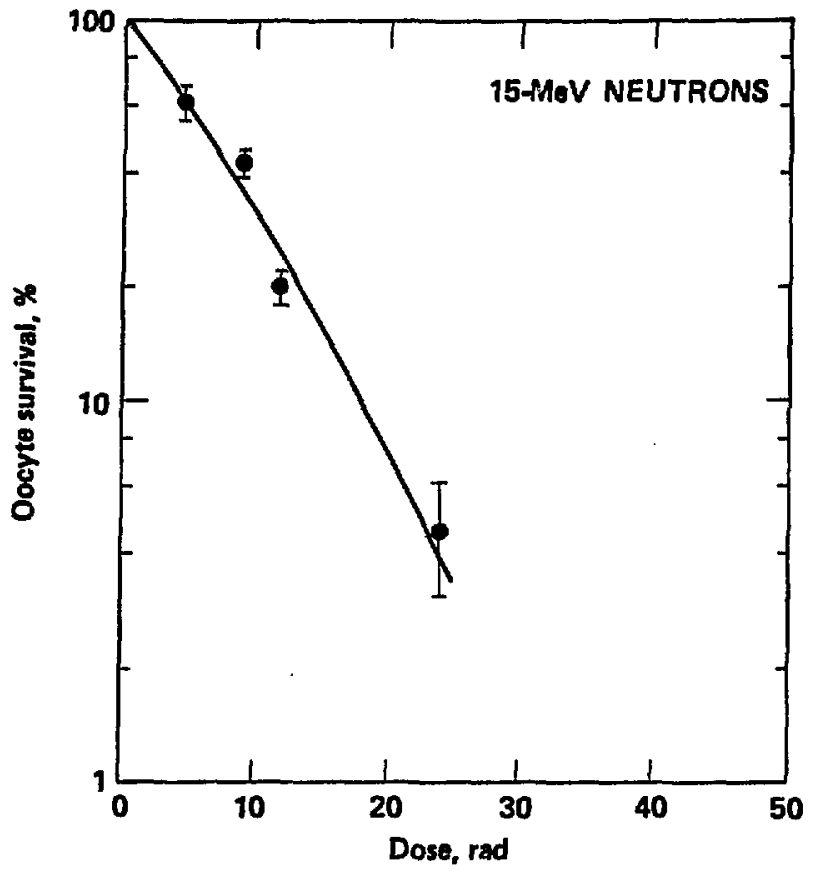

FIG. 3.15. Oocyte survival following 15-MeV neutron irradiation at 18 days of age. Error bars are \pm 1 SEM. 


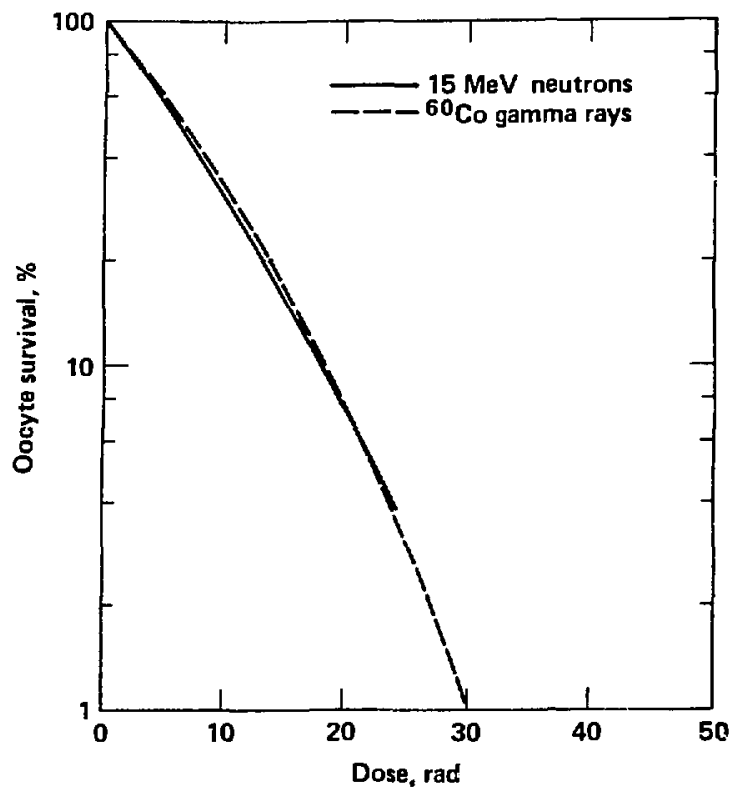

FIG. 3.16. Comparison of gocyte survival following irradiation with $15-\mathrm{MeV}$ neutrons and with ${ }^{6} \mathrm{Co}$ gamma rays. 


\section{DISCUSSION}

Effect of Dose on Neutron RB̈E

An increase in neutron RBE with decreasing dose is observed in numerous endpoints (see Chapter 2). It is generally the result of . decreasing gamma-ray effectiveness at low doses, while neutron effectiveness remains constant. The oocyte results also show this trend and are well fitted by linear dose-response functions for 0.43-MeV and fission neutrons and by linear-quadratic dose-response functions for ${ }^{60} \mathrm{Co}$ gamma rays. This results in an increasing RBE with decreasing dose. However, in contrast to other radiosensitive biological endpoints (see Referencs $4,6,7$ ), the mouse oocyte system used here yields neutron RBEs that are much lower. Even with 0.43-MeV neutrons, which are of greatest biological effectiveness, the RBE for oocyte killing reached only 1.8 at 1 rad, compared to, for example, about 60 at this dose for mouse cataracts (7).

0ocyte killing with ${ }^{252} \mathrm{Cf}$ fission neutrons appears unusual in that these neutrons are less, not more, effective than gamma-rays (Fig. 3.12). The RBE is less than 1. As for 0.43-MeV neutrons, the RBE for fission neutrons increases with decreasing dose, but differs in being a factor of about 2 lower at all doses tested.

RBEs less than unity have been reported for damage to membrane-type structures. The release of enzymes from isolated rat spleen lysosomes (56) exposed in vitro to equal doses of 7-MeV neutrons or 7-MeV electrons gave RBE less than 1. That is, more enzyme was released to the medium after electron irradiation than 
after neutron irradiation. It is unlikely that lysosomes play an important role in oocyte radiation killing, since the number of lysosomes per oocyte is small (see Fig. 4.4), and their number does not appear to reflect the variations in radiosensitivity with age. However, the observation that membrane damage results in very low neutron RBEs (in some cases less than unity) is an extremely interesting similarity with the results for mouse oocyte killing obtained here. It is of special significance that such low RBEs are not found for radiation cell killing in rapidly dividing systems, where nuclear damage is implicated. Further discussion on the radiosensitive target for oocyte killing will be made in Chapter 4.

As noted above for neutrons with energy of $15 \mathrm{MeV}$, the dose-response was not significantly different from that for ${ }^{60} \mathrm{Co}_{0}$ gamma rays at all doses tested (Fig. 3.16 ). The RBE is therefore unity. The fitted dose-response curves do suggest, however, that 15-MeV neutrons maj be slightly more effective at lower doses and less effective at higher doses than ${ }^{60}$ Co gamma rays. The RBE for 15-MeV neutrons inferred from the fitted survival curves in Fig. 3.16 is only 1.1 at low doses and decreases with increasing dose to slightly less than one at larger doses. It is interesting to note the striking similarity in dose-response with two radiations of such highly contrasting LET. Cobalt-60 gamma rays have average LET of about $0.24 \mathrm{keV} / \mathrm{\mu m}$ whereas $15-\mathrm{MeV}$ neutrons have LET some 50 fold higher, about $12 \mathrm{keV} / \mu \mathrm{m}$. This extraordinary insensitivity of mouse oocyte killing to changes in LET is one of the unusual features of this biological system which results in low RBE values. 
Effect of Neutron Energy on Neutron RBE

In addition to neutron dose, neutron energy also influences the RBE. For most endpoints RBEs yary with neutron energy in a predictable manner, as can be seen in Fig. 2.11. The trend is for a maximum RBE at a few hundred keV and lower values both above and below this, correlating well with the effective LET of proton recoils in soft tissue (12). As energy is increased, a minimum RBE appears * to be reached at about 10 to $20 \mathrm{MeV}$, followed by a slight increase at higher energies.

When the oocyte RBE data are plotted against neutron energy, a similar trend is seen (see Fig. 3.17). However, important differences are observed. First, the RBE is lower at all energies tested, probably reflecting the high gamma-ray sensitivity of this endpoint. Second, the minimum RBE appears to be at about $2 \mathrm{MeV}$ rather than at 10 to $20 \mathrm{MeV}$. Third, the RBE for fission neutrons is about 1 at very low doses and becomes less than 1 at larger doses. The possible reasons for these differences are discussed further in Cinapter 4.

Effect of the Animal's Age on Neutron RBE for Oocyte Killing

Neutron RBEs for mouse oocytes are also affected by the animal's age at time of exposure. Shown in Fig. 3.18 are RBEs for mouse oocytes irradiated 11 days or 17 days postconception, or at 14 days postpartum. It is important to note that the large decrease in RBE between 17 days postconception ( 2 days before birth) and 14 days after birth is due actually to a relatively greater increase in 


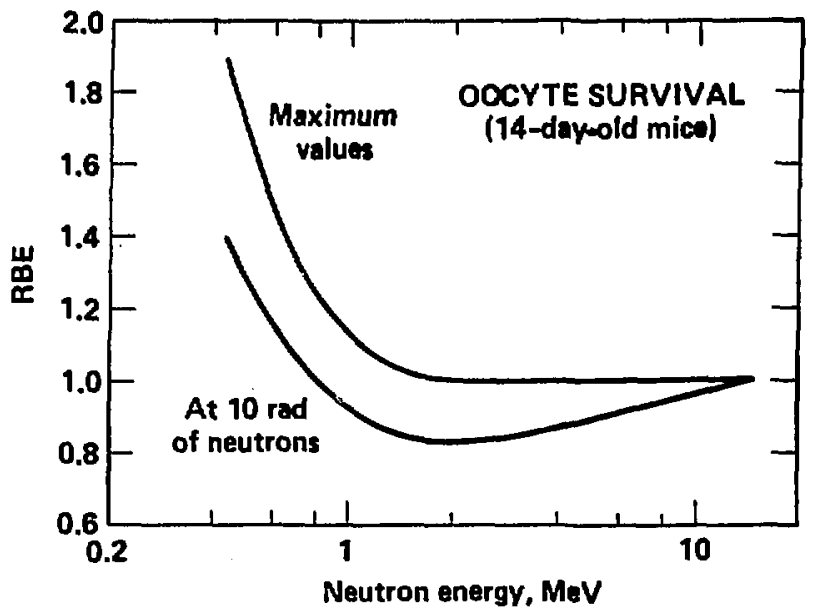

FIG. 3.17. The variation in neutron RBE with neutron energy for mouse oocytes. Maximum values are obtained at very low doses where both survivi? curves (for neutrons and gama rays) become exponential. 


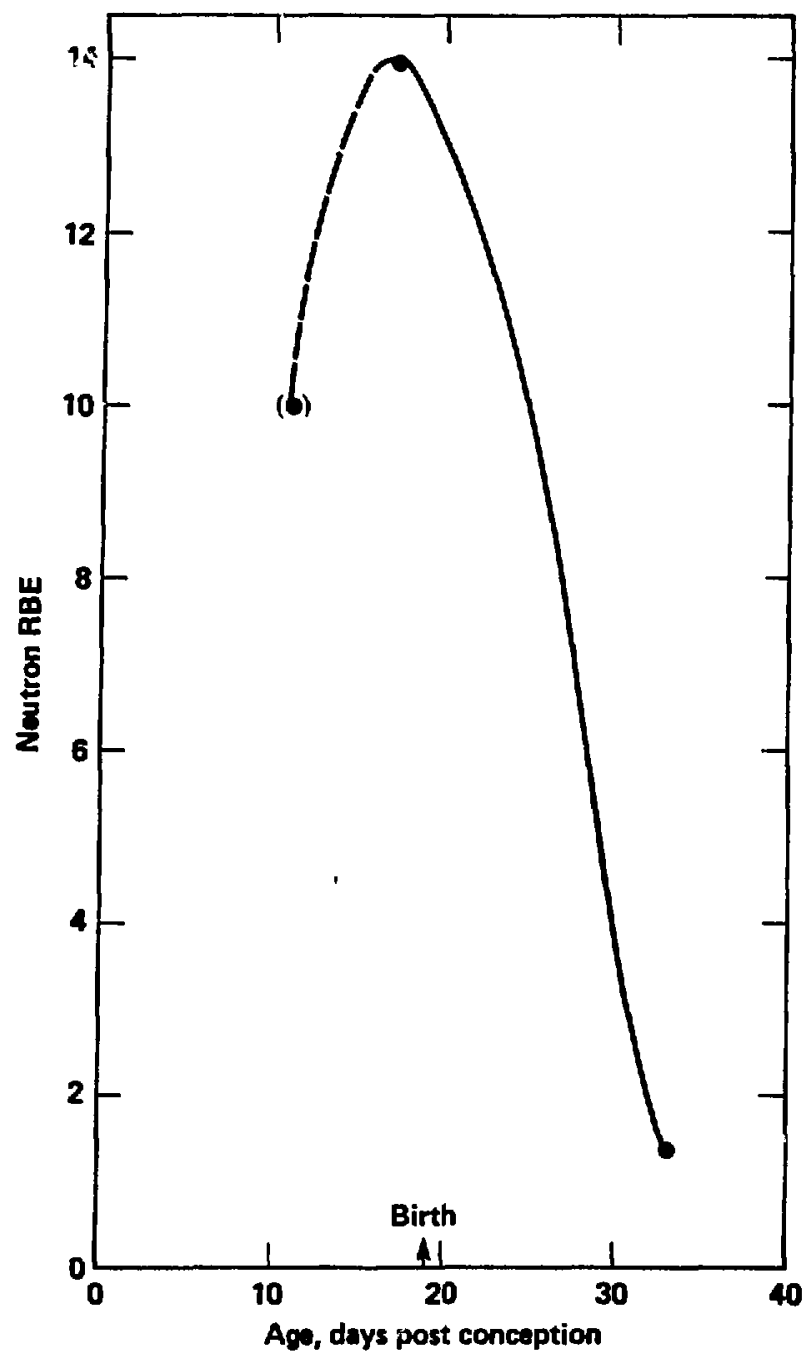

FIG. 3.18. Variation in neutron RBE for mouse oocyte killing with the animal's age at exposure. The three ages 111 days and 17 days posi conception, and 14 days post natal) were selected as described in text. RBEs are evaluated at 10 rad of neutrons. The RBE point at 11 days post conception (in parenthesis) is uncertain since adequate gamma-ray data are not available for this age period. 
gamma-ray sensitivity than in neutron sensitivity. For example, the $\mathrm{LD}_{50}$ for oocyte killing with gamma rays given 2 days before birth is about 200 rad, while at 14 days after birth it is only 8 rad; the $L_{50}$ for gamma rays changes 25 fold! With neutrons, however, the $\mathrm{LD}_{50}$ varies by a factor of only adout 3. A possible explanation for the extremely high sensitivity to gamma rays at 14 days of age (which results in low-neutron RBEs) is discussed in Chapter 4. Neutron RBEs for mouse oocyte killing are compared in Fig. 3.19 to RBEs from other radiosensitive biological endpoints. It is interesting to note that the RBE for oocyte killing just prior to birth is very much like those observed for most other endpoints (garma-ray sensitivity also compares well to other cell-survival studies, with $L D_{50}$ about 200 rá:?, while in 14-day-old mice it is much lower. At about 11 days postconception the germ-ce 11 pool is being formed by rapid oogonial divisions, and therefore radiation exposure at this time might be expected to result in reproductive cell death (lethal impairment of the cell's ability to divide) of the oocyte's precursors. Hence, the neutron RBE might be expected to be similar to those determined for other rapidly dividing cells. This appears to be the case (Fig. 3.19), although not enough ${ }^{60} \mathrm{Co}_{0}$ gamma-ray data are available for this age period to obtain precise RBE values. 


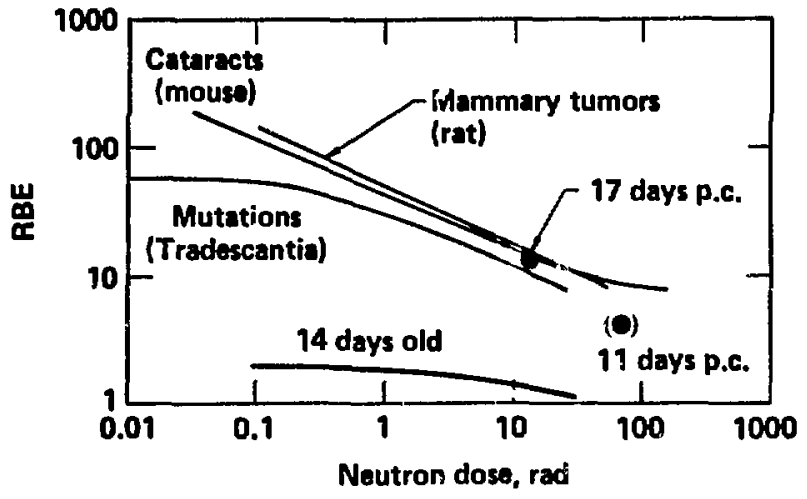

FIG. 3.19. Neutron RBEs for mouse oocytes compared to neutron RBES for other radiosensitive biological systems. The RBEs for prenatal exposures were evaluated at the $\mathrm{LD}_{50}$ for oocyte killing; as in Fig. 3.18, the 11-day post conception (p.c.) value must be considered an estimate only (see text for details). 
CONCLUSIONS

The very great radiosensitivity of mouse oocytes makes these cells of particular interest for studying neutron RBE at very low doses, in the exposure range of most practical significance to radiological health protection. Very few neutron RBE experiments have been reported for endpoints that yield quantifiable effects at doses in the one-rad range; hence, the oocyte data are of particular importance. The experimental results presented here clearly demonstrate that neutron RBEs for mouse oocyte killing do not reach the high values (100 or greater) observed with other low-dose studies. In fact, neutron RBEs for mouse oocjtes appear unusually low during the highly radiosensitive period of these cells, with maximum values of only about 1.9 .

Neutron RBE in the oocyte system is influenced by both neutron dose and energy, as in most biological systems. The highest value was obtained with low doses of $0.43-\mathrm{MeV}$ neutrons. The observation that fission neutrons give RBEs less than unity for mouse oocyte killing is believed to reflect an unlisual sensitive target which, because of its geometrical configuration, al lows both gamma rays and $3_{H}$ beta rays to be relatively more effective than fission neutrons. This target is thought to be the plasma membrane and is discussed in Chapter 4.

The mouse's age at exposure also affects the neutron RBE for oocyte killing. LOW RBE values are obtained during the highly 
radiosensitive juvenile period (e.g., in 14-day-old mice), while exposure just prior to birth yields RBEs that are consistent with those observed for many other biological effects. 


\section{THE RADIOSENSITIVE TARGET IN MOUSE OOCYTES}

\section{INTRODUCTION}

We have seen that the oocyte system has unusual dose-response characteristics. Does this reflect an unusual subcellular target? Certainly the oocyte differs in certain ways from other body cells both in structure and function, but our present knowledge of cellular radiation damage at the very low doses used here is not able to satisfactorily explain the findings in Chapter 3 . In an attempt to understand the mechanism involved in radiation oocyte killing, the target size and the microdistribution of energy deposition must be carefulty examined.

The target size, and to some extent target shape, can be estimated from survival curves and published microdosimetric parameter values. Figure 4.1 gives functions for event (energy-absorption events) frequency vs sphere diameter obtained by exrosing specially designed tissue-equivalent proportional counters co various radiation types $(29,57)$. The event frequency is tire number of charged particle traversals per unit of absorbed dose and includes all energy deposition in the detector volume. The sphere diameter is that of a unit density tissue sphere and was obtained by adjusting the tissue-equivalent gas pressure in the proportional counter to reflect spherical volumes that are similar to cells and organelles. The event frequency for a biological target can be estimated (for radiations producing exponential survival curves) by assuming an average of 1 event per target at the $\mathrm{D}_{37^{\circ}}$. Since 


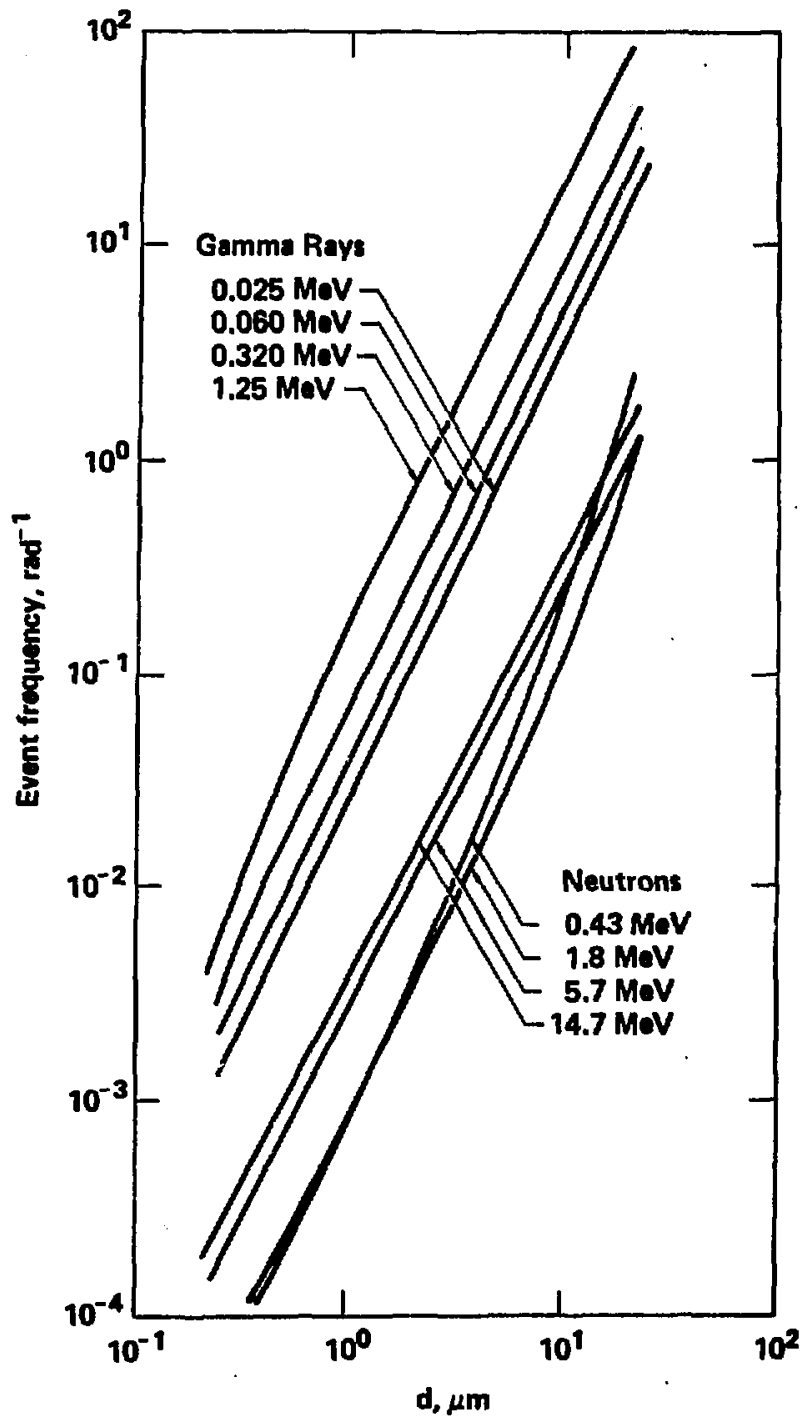

FIG. 4.1. Event frequency for various radiations as a function of tissue sphere dianeter. See text for explanation. Redrawn from $(29,57)$. 
deactivation of a sensitive biological target may require some minimum energy absorption, the event frequency (and target size estimated from it using the data in Fig. 4.1) determined in this way represents the smallest possible target and is therefore probably a slight underestimate.

\section{TARGET SIZE ESTIMATION WITH NEUTRONS}

For $0.43-\mathrm{MeV}$ and fission neutrons, the event frequency was estimated from the oocyte data by taking the reciprocal of the $\mathrm{D}_{37}$. The data in Fig. 4.1 were then used to obtain the target (tissue sphere) diameters for immature mouse oocytes listed in Table 4.1. A target diameter of about 9 um obtained for $0.43-\mathrm{MeV}$ neutrons is larger than the nuclear diameter $(26 \mu \mathrm{m})$ and is slightly smaller than the size of the entire immature mouse oocyte $(10-12 \mu \mathrm{m})$. This means that for $0.43-\mathrm{MeV}$ and fission neutrons a "hit" almost anywhere in the cell during this highly sensitive period results in death to the oocyte! This is not true for $15-\mathrm{MeV}$ neutrons or ${ }^{60}$ Co gamma rays, however, where multi-hit cell killing is suggested by the survival-curve shapes.

When these same target size calculations are made for oocytes exposed on the 11th or 17th postconception days, different results are obtained. The target diameter at these ages ranges from 4 to $6 \mu \mathrm{m}$ (see Table 4.2), similar to the size of an oocyte nucleus. The overall picture emerging from these target size determinations is that the "target" changes with the animal's age and that during periods of low radiosensitivity the target corresponds to the nucleus 
TABLE 4.1. Estimated radiosensitive target dianeters in mouse oocytes.

\begin{tabular}{lrcc}
\hline $\begin{array}{l}\text { Neutron } \\
\text { energy }\end{array}$ & $\begin{array}{c}\mathrm{D}_{37} \\
(\mathrm{rad})\end{array}$ & $\begin{array}{c}\text { Event } \\
\text { frequency } \\
\left(\mathrm{rad}^{-1}\right)\end{array}$ & $\begin{array}{c}\text { Target } \\
\text { diameter } \\
(\mu \mathrm{m})\end{array}$ \\
\hline & & & \\
$0.43 \mathrm{MeV}$ & 5.5 & 0.182 & 9 \\
Fission & 11.5 & 0.087 & 8.5 \\
\hline
\end{tabular}

TABLE 4.2. Variation in target dianeter with mouse age for 0.43-HeV neutrons.

\begin{tabular}{cccc}
$\begin{array}{c}\text { Mouse } \\
\text { age }\end{array}$ & $\begin{array}{c}\mathrm{D}_{37} \\
(\mathrm{rad})\end{array}$ & $\begin{array}{c}\text { Event frequency } \\
\left(\mathrm{rad}^{-1}\right)\end{array}$ & $\begin{array}{c}\text { Target diameter } \\
(\mu \mathrm{m})\end{array}$ \\
\hline 11 days p.c. & 75 & .013 & 4 \\
17 days p.c & 17 & .059 & 6 \\
14 days old & 5.5 & .382 & 9 \\
\hline
\end{tabular}


while during high radiosensitivity it corresponds to the entire cell. It has been noted that blood concentrations of Follicle Stimulating Hormone (FSH) show a distinct peak during the period of greatest oocyte radiosensitivity in juvenile mice, however inducing such sensitivity changes by administering hormone has not been successful $(58,59)$.

For $0.43-\mathrm{MeV}$ and possibly fission neutrons as well, survival curves are exponential, indicating single-hit kinetics, and therefore cell killing should result from traversal of a single proton recoll. The average proton recoil track lengths for the neutron radiations used here are about $3 \mathrm{~mm}$ for $0.43-\mathrm{MeV}, 30 \mu \mathrm{m}$ for fission, and 625 $\mu \mathrm{m}$ for $15-\mathrm{MeV}$ neutrons. It should be noted that the track length from a $0.43-\mathrm{MeV}$ neutron is less than either the cell or nuclear diameter, while the track from an average fission or 15-MeV neutron is considerably greater than cell dimensions. This observation may have important implications for the interpretation of dose-response curves. Shown in Table 4.3 are the average energies deposited per nucleus and per cell at the $D_{37}$ for the three different neutron energies used here. Tha. indicate that the energy deposited in the total cell is approximately the same for all three, whereas large differences are observed for smaller volumes such as the cell nucleus.

\section{APPARENT INCONSISTENCIES}

Although target sizes determined from $0.43-\mathrm{MeV}$ and from fission neutrons implicate the entire cell as sensitive target during the highly sensitive period, results from ${ }^{3} H$ beta rays and ${ }^{60}$ Co gamma 
TABLE 4.3. Average energy deposition at $D_{37}$ in the nucleus and total cell for the neutron radiations used.

Radiation $\frac{\text { Energy deposited* (keV) }}{\text { in nucleus in cell }}$

$\begin{array}{lrr}0.43-\mathrm{MeV} \text { neutrons } & 215 & 215 \\ \text { Fission neutrons } & 90 & 180 \\ 15-\mathrm{MeV} \text { neutrons } & 126 & 210\end{array}$

* Calculated from LET and average recoil proton ranges in unit density material. .

rays do not support such a conclusion. Target size estimates with low-LET radiation, such as gamma rays, will not discriminate between small solid spheres and more diffuse structures of equal total volume. These radiations provide information only on target volume, not shape. On the other hand, neutron radiation, through the bullet-like proton recoils, will only reflect the target's cross-sectional area. Studies with both types of radiations, however, can reveal shape as well as size. This was well pointed out by Schneiderman and Hofer (60) who interpreted experimental results on radiation-induced division delay as indicating a nuclear membrane target. Table 4.4 gives estimated target volumes and areas for the radiations used here. It is apparent that the cross-sectional areas for neutrons are consistent for all energies tested, and are almost equal to the cross-sectional area of the cell. However, results with 
TABLE 4.4. Oocyte target size estimates for the radiations used in this study.

\begin{tabular}{lcccc}
\hline $\begin{array}{l}\text { Radiation } \\
\text { type }\end{array}$ & $\begin{array}{c}\text { Number* } \\
\text { of hits } \\
\text { required }\end{array}$ & $\begin{array}{c}\text { Diameter** } \\
\text { "per hit" } \\
(\mu \mathrm{m})\end{array}$ & $\begin{array}{c}\text { Targett } \\
\text { volume } \\
(\mu \mathrm{m}) 3\end{array}$ & $\begin{array}{c}\text { Target† } \\
\text { area } \\
(\mu \mathrm{m}) 2\end{array}$ \\
\hline${ }^{60}$ Co ganma rays & 10 & 0.9 & 3.8 & 6.4 \\
${ }^{3}$ H beta rays & 1 & 2.4 & 8.0 & 4.8 \\
0.43-MeV neutrons & 1 & 9 & 382 & 64 \\
Fission neutrons & 1 & 8.5 & 322 & 57 \\
15-MeV neutrons & 3 & 5 & 198 & 59 \\
\hline
\end{tabular}

* From survival curve analysis

** Obtained from the event frequency (reciprocal of the $D_{37}$ ) and Fig. 4.1.

+ Derived from the diameter per hit and the number of hits required, e.g., for ${ }^{60}$ Co gamma rays the target area is $10 \pi(\mu \mathrm{m})^{2}$.

${ }^{60}$ Co gamma rays and ${ }^{3} \mathrm{H}$ beta rays, yielding target sizes an order of magnitude smaller, require further explanation. Another, but related, inconsistency appears when the microdosimetric quantity $\zeta$ (a function of target size) is estimated from the acute ${ }^{60}$ Co gamma-ray survival curve. A value of 37 rads is obtained, which is in great contrast to estimates from microdosimetric measurements in volumes as large as mouse oocytes (48) where a $\zeta$ of only about $0.1 \mathrm{rad}$ is found (see Fig. 4.2).*

* The data in Fig. 4.2 are plotted from microdosimetric results obtained by Elett and Braby (48) for tissue-equivalent spheres between $0.6 \mu \mathrm{m}$ and $5 \mu \mathrm{m}$ diameter. Extrapolation to larger sphere diameters, such as mouse oocytes, is considered justified since the data are linear on a $\log -\log$ plot. 


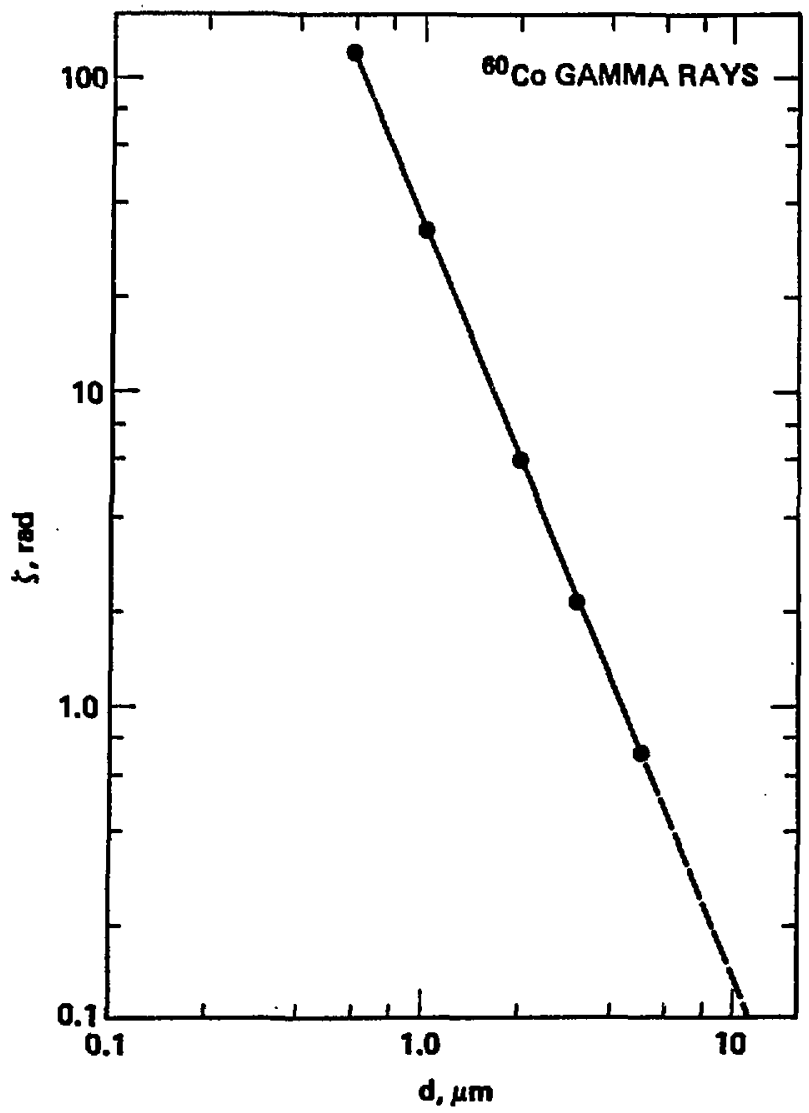

FIG. 4.2 Dose average of the specific energy ( $\zeta$ ) imparted by single 60 Co gamma-ray events in tissue-equivalent spheres of diameter (d). The value of $\zeta$ is a function of both radiation type and sensitive target volume. The curve is extrapolated (dashed line) to diameters similar to those of immature mouse oocytes $(212 \mu \mathrm{m})$. Plotted from tabulated data given by (48). 


\section{RECONCILIATIONS}

An effort is made here to resolve these apparent inconsistencies. Various subcellular organelles are considered in turn as possibly being the target. An electron micrograph of a typical immature mouse oocyte, the cell used in these experiments, is shown in Fig. 4.3 and will be referred to during the following discussion.

\section{Cell Nucleus}

In most biological systems the cell nucleus is believed to be the sensitive target in radiation cell killing, (see, e.g., 61-64). However, for immature mouse oocytes during their highly radiosensitive period (in the juvenile mouse) the nucleus does not seem to be involved in cell killing. For one thing, the estimated size of the sensitive target (Table 4.4) does not correspond with the size of the oocyte nucleus. Also, ${ }^{3} \mathrm{H}$ confined to the oocyte nucleus, having been administered as ${ }^{3} \mathrm{H}$-thymidine during fetal development and incorporated into oogonial DNA (65), is clearly less effective in oocyte killing than ${ }^{3} \mathrm{H}$ more randomly distributed, having been administered as ${ }^{3} \mathrm{HOH}$ (41). The nucleus, therefore, does not appear to be the sensitive target in immature oocytes of juvenile nice.

\section{Endoplasmic Reticulum}

Electron microscopic data argue against the endoplasmic reticulum as sensitive target. This structure is not a prominent 


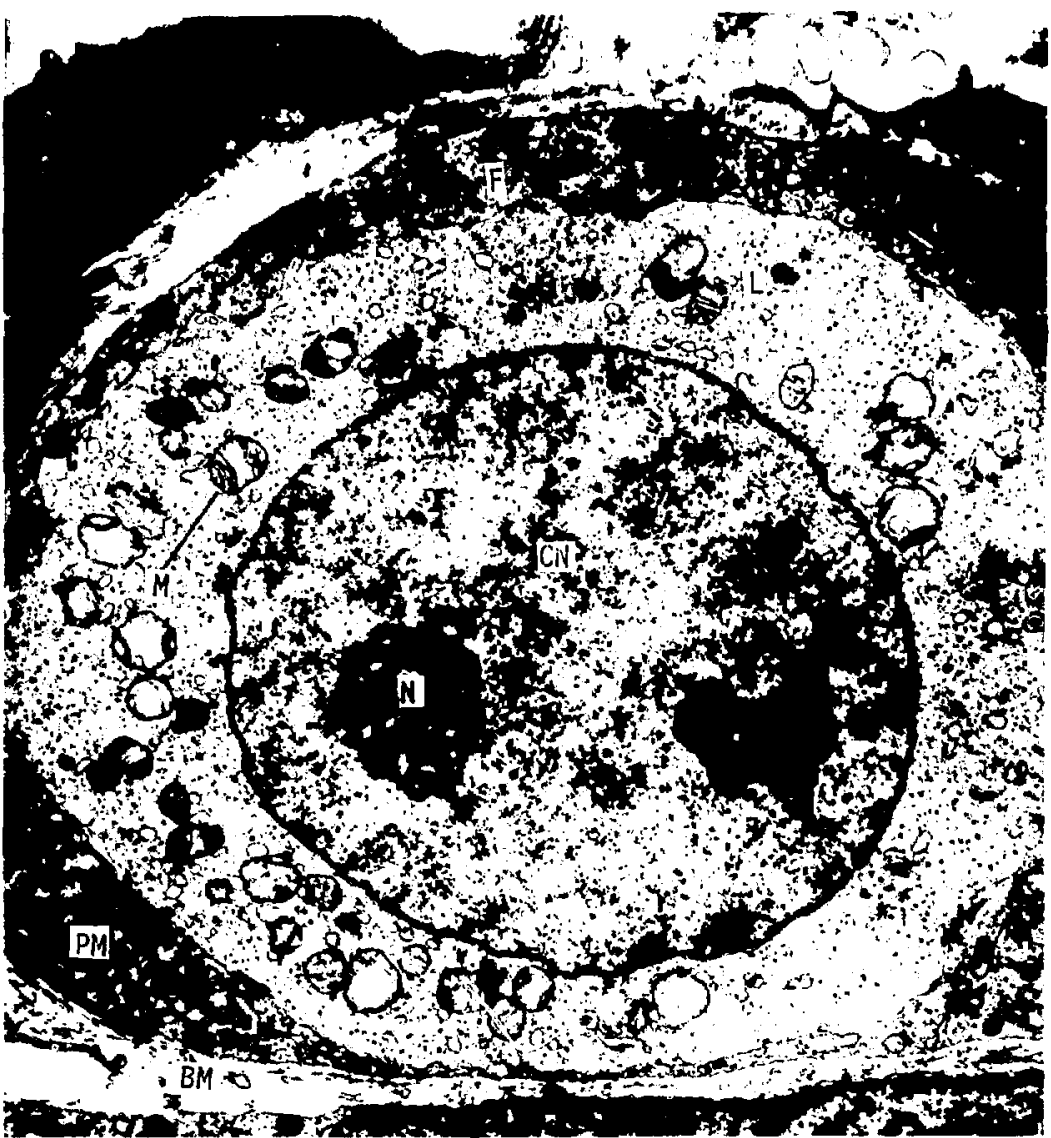

FIG. 4.3. Electron micrograph of an immature mouse oocyte in a 14-day-old albino Swiss mouse. Identifiable structures and organelles are indicated; cell nucleus (CN), nucleolus (N), mitochondrion (M), lysosome (L), plasma membrane (PM), Golgi complex $(G)$, follicle cell (F) and basement membrane (BM). The oocyte is about $12 \mu \mathrm{m}$ in diameter. Reproduced by permission from (97). 
feature of the mouse oocyte (Fig. 4.3). A special form of smooth ER, the annulate lamellae, have been observed in oocytes (also in other cell types). The intimate connection of these organelles with the nuclear membrane makes them of special interest. Their function is not well understood. However, it does not appear that the mouse oocyte contains enough of these to possibly account for the large target size obtained with $0.43-\mathrm{MeV}$ neutrons. None are seen in Fig. 4.3.

\section{Lysosomes}

These microscopic, membranous sacs do not fit the target requirements given in Table 4.4. First, there are not enough lysosomes in the small oocyte (Fig. 4.3) to account for the large target size obtained with $0.43-\mathrm{MeV}$ neutrons, and second, they are too small to account for the target volumes observed with ${ }^{3} \mathrm{H}$ and ${ }^{60} \mathrm{Co}$ radiation.

Mitochondria

The oocyte may contain enough mitochondria to account for the large target size (cross-sectional area) observed with neutrons (Fig. 4.3). However, the volume of a single mitochondrion is much smaller than the target volume determined with ${ }^{3} \mathrm{H}$ and ${ }^{60} \mathrm{Co}$ radiation. So if the target were mitochondrial, it would require deactivation of more than one mitochondrion for oocyte killing, and this is inconsistent with the observed exponential survival curve for ${ }^{3} \mathrm{H}$ beta rays. 


\section{Plasma Membrane}

This organelle appears to satisfy the requirements imposed by the $0.43-\mathrm{MeV}$ neutron data, i.e., target area is aimost equal to the cross-sectional area of the cell. The volume of the plasna membrane $\left(24.5 \mu \mathrm{m}^{3}\right.$ ) also satisfies the demands of the ${ }^{60}$ Co gamma-rays data. The plasma membrane will, therefore, be considered in more detail.

Since the plasma membrai:s is a very thin spherical shell of about 100-A thickness, energy deposition from ionizing particles traversing it car, be very different from that in a solid tissue sphere of equal total volume. The plasma membrane geometry results in energy absorption characteristics that will now be analyzed. Tables 4.5 and 4.6 compare the energy transfer characteristics (66) of the neutron radiations used here and their respective proton recoil track dimensions (67). Figure 4.4 shows the dimensions of these tracks in relation to those of the cell plasma membrane. It is interesting that the diametens of these tracks range from 100-1000 A (65) (very small fractions, $0.01 \%$ to $0.1 \%$, of the cell diameter) and yet single tracks may cause cellular death: A neutron with $0.43 \mathrm{MeV}$ of energy produces (through its proton recoil) over twice the number of ionizations in the plasma membrane (in one traversal) than does an averaye fission neutron. However, proton recoils from fission neutrons are very likely to pass through the entire cell, thereby hitting the plasma membrane twice and producing nearly the same amount of ionization in the cotal membrane. On the other hand, a solid tissue sphere of the same total volure (volume of 
TABLE 4.5. Energy transfer

characteristics of the neutrons used.

\begin{tabular}{lcc}
$\begin{array}{l}\text { Neutron } \\
\text { energy } \\
(\mathrm{MeV})\end{array}$ & $\begin{array}{c}\text { LET* } \\
(\mathrm{keV} / \mu \mathrm{m})\end{array}$ & $\begin{array}{c}\text { Ionization } \\
\text { (ion pairs } / 100 \mathrm{~A})\end{array}$ \\
\hline 0.43 & 72 & 21 \\
1.8 & 30 & 9 \\
15 & 12 & 3.5 \\
\hline * From Ref. 66. &
\end{tabular}

TABLE 4.6. Track dimensions of recoil protons.

\begin{tabular}{lcc}
\hline $\begin{array}{l}\text { Meatron } \\
\text { energy } \\
(\mathrm{MeV})\end{array}$ & $\begin{array}{c}\text { proton } \\
\text { energy } \\
\text { (MeV) }\end{array}$ & $\begin{array}{c}\text { Track } \\
\text { diameter } \\
\text { (A) }\end{array}$ \\
\hline & & \\
0.43 & 0.215 & 100 \\
1.8 & 0.9 & 700 \\
15 & 7.5 & 1000 \\
\hline
\end{tabular}

entire cel?) would receive from a proton recoil of a $0.43 \mathrm{MeV}$ neutron only about half as much energy as it would from a proton recoil of a 1.8-MeV neutron (average fission neutron). 


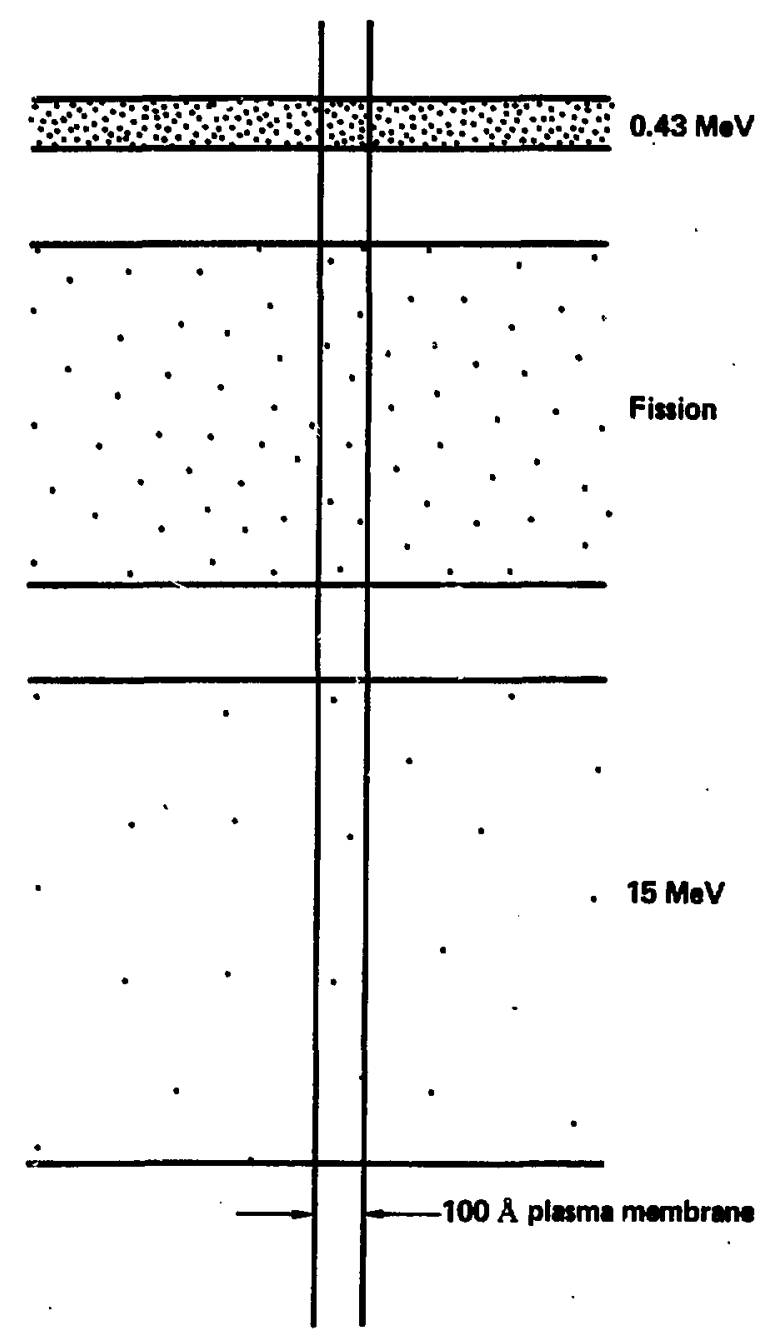

FIG. 4.4. Dimensions of proton recoil tracks traversing the plasma membrane. Each dot in the tracks represents an ion pair. The incident neutron energies are indicated. 
Proton recoils from 15-MeV neutrons produce considerably fewer ionizations in the plasma membrane, requiring several cell-traversals to produce the equivalent of that from single proton traversals from $0.43-\mathrm{MeV}$ or fission neutrons. For $15-\mathrm{MeV}$ neutrons the required number of cell-traversals for oocyte kiliing was estimated by three different methods, as outlined in Table 4.7, and it is seen that about 3 traversals are needed.

Gamma rays produce more randomly scattered ionizations, resulting in considerably lower ion-density. Hence, indirect action effects mediated by radicals produced in the radiation hydrolys is of water (68) are likely to be relatively more important in the case of gamma rays than are direct action effects. However, the diffusion distances of the major hydrolysis products, shown in Table 4.8 (69)

TABLE 4.7. Number of recoil proton cell traversals required for oocyte killing by 15-MeV neutrons estimated by 3 methods.

Method

Required number

Event frequency at $D_{37}$ in

12-um diameter tissue sphere 23

For $600 \mathrm{eV}$ imparted to plasma membrane 23 
TABLE 4.8. Diffusion distances for radiolys is products of water following ${ }^{60}$ Co irradiation.

\begin{tabular}{lc}
\hline $\begin{array}{l}\text { Radiolysis } \\
\text { product }\end{array}$ & $\begin{array}{c}\text { Diffusion } \\
\text { distance } \\
(\mathrm{A})\end{array}$ \\
\hline $\mathrm{e}_{\overline{\mathrm{aq}}}$ & 100 \\
$\mathrm{OH} \cdot$ & 50 \\
$\mathrm{H} \cdot \mathrm{H}$ & 50 \\
$\mathrm{H}_{2} \mathrm{O}_{2}$ & 1000 \\
\hline
\end{tabular}

are similar to the track diameters of neutron-induced proton recoils (see Table 4.6). Therefore, an ion cluster produced by ${ }^{60} \mathrm{Co}_{\mathrm{w}}$ will exert its damaging affect over distances similar to proton track diameters, but at much lower intensity.

The sigmoidal survival curve for ${ }^{60}$ Co gamma rays indicates multihit iell-killing kinetics (Fig. 4.5). The number of hits (primary ionizations) in the membrane required to kill the oocyte are est imated by the same methods used for $15-\mathrm{MeV}$ neutrons, with the exception that here membrane volume is used rather than the cross-sectional area of the cell. Results are listed in Table 4.9 and show that about 10 hits are needed.

As the level of radiation effect probably reflects the energy absorbed in the sensitive target rather than the overall tissue dose (70), the absorbed energy required in the sensitive target for cell killing shouid be about equal for ${ }^{3} \mathrm{H}$ bewa particles and $0.43-\mathrm{MeV}$ and fission neutrons and, with appropriate modification, also for 


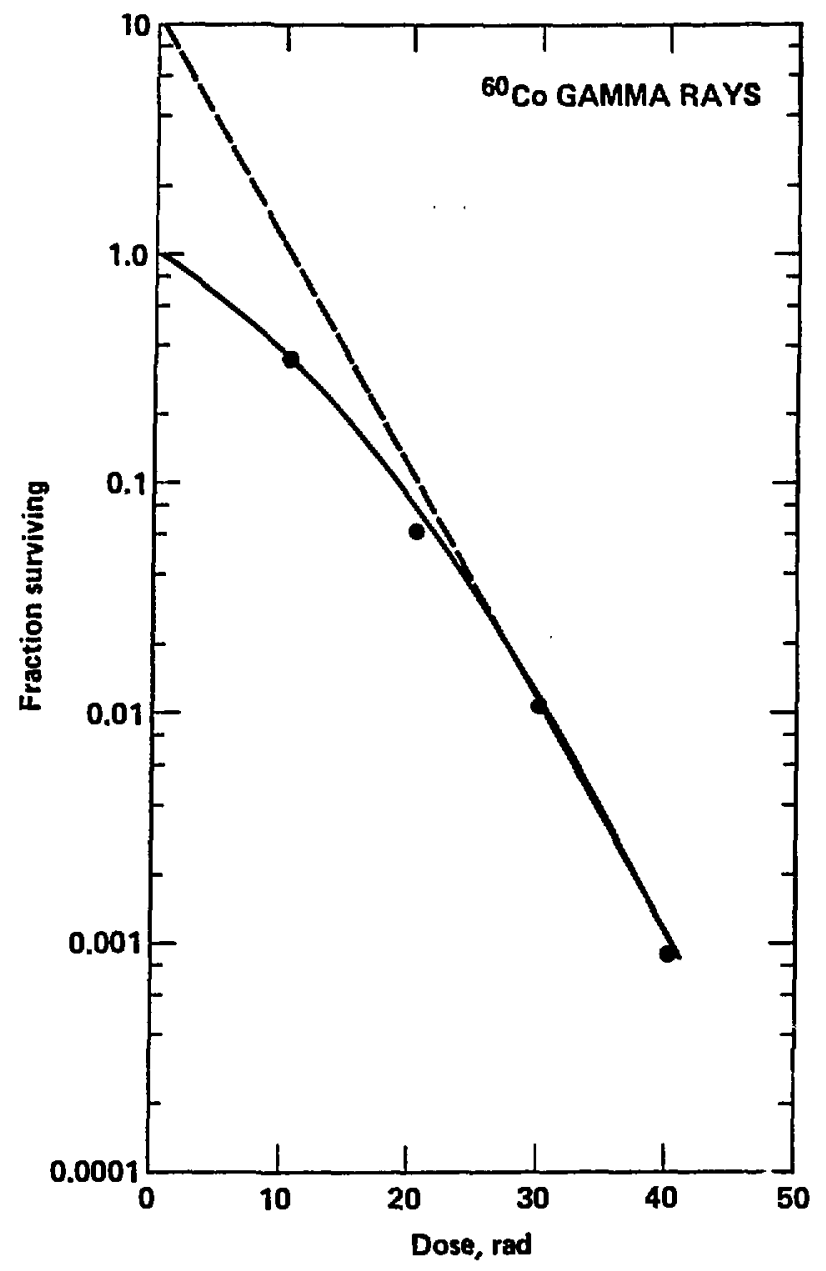

FIG. 4.5. Extragolation-number estimated from mouse oocyte survival curve for acute ${ }^{60}$ Co gamma rays (see text). 
TABLE 4.9. Nunber of ionizations ("hits")

recuired in volume of plasma membrane for oocyte killing by ${ }^{60}$ Co gamma rays, estimated by three methods.

Method

Event frequency at $\mathrm{D}_{37}$ in

4.5- $\mu \mathrm{m}^{3}$ tissue sphere

For $600 \mathrm{eV}$ imparted to

plasma niembrane

Extrapolation number

${ }^{60}$ Co gamma rays and 15-MeV neutrons. Comparisons are made in Table 4.10 for the plasma membrane. It is seen that the total energies absorbed from the various radiation types agree we11, about $600 \mathrm{eV}$. For 15-MeV neutrons total energy deposited in the membrane was determined by multiplying the energy deposited per traversal and the number of traversals at the $D_{37}$. Cobalt-60 garma rays deposit energy in random clusters of somewhere between 34-100 eV each (71); hence, for energy absorption in the membrane values about 10 times these are "expected from 10 hits in the membrane volume. For fission and $0.43-\mathrm{MeV}$ neutrons (requiring only one proton recoil to $1: i l l$ the cellj the energy deposition was determined by multiplying the membrane thickness by the track-averaged LET of the proton recoil. 
TABLE 4.10. Energy imparted to plasma membrane at $D_{37}$ by the radiations used.

\begin{tabular}{lccc}
\hline $\begin{array}{l}\text { Radiation } \\
\text { type }\end{array}$ & $\begin{array}{c}\text { Number of } \\
\text { hits required }\end{array}$ & $\begin{array}{c}\text { Energy } \\
\text { per event }\end{array}$ & $\begin{array}{c}\text { Total } \\
\text { energy }\end{array}$ \\
\hline${ }^{60}$ Co gamma rays & 10 & $34-100 \mathrm{eV} *$ & $340-1000 \mathrm{eV}$ \\
${ }^{3} \mathrm{H}$ beta rays & 1 & $100-5700 \mathrm{eV} * *$ & $100-5700 \mathrm{eV}$ \\
$0.43-M e V$ neutrons & 1 & $700 \mathrm{eV}$ & $700 \mathrm{eV}$ \\
Fission neutrons & 1 & $600 \mathrm{eV}$ & $600 \mathrm{eV}$ \\
$15-M e V$ neutrons & 3 & $200 \mathrm{eV}$ & $600 \mathrm{eV}$ \\
\hline
\end{tabular}

* Depends on whether a single ion-pair or an entire ion-cluster deposits it is energy in the plasma membrane.

** Possible range of deposited energy depends on angle at which the beta ray traverses the plasma membrane.

Although the average ellergy deposition per membrane traversal is relatively straightforward to calculate, the probability that any given hit in the cell will also result in membrane traversal is a more complicated problem for neutrons of low to moderate energy. Proton recoils from $0.43-\mathrm{MeV}$ neutrons have average ranges in tissue of only about $3.0 \mu \mathrm{m}$ (considerably less than the cell diameter), therefore a fraction of the hits in the cell will not traverse the membrane at all. This fraction is estimated by the following method.

An equally spaced array of points is chosen representing locations of random neutron-proton interactions. Since interactions with hydrogen, at these neutron energies, are almost totally through 
elastic collisions (72), the mean recoil energy $\left(\bar{E}_{r}\right)$ can be calculated from

$$
E_{r}=E_{n} \frac{2}{\left(M_{n}+M_{H}\right)^{2}}=E n / 2,
$$

where $E_{n}$ is the neutron energy, $M_{n}$ is the neutron mass and $M_{H}$ the proton mass. It follows that $\bar{E}_{r}$ is equal to half the incident neutron energy. For neutron energies below about $15 \mathrm{MeV}$ the recoil protons are scattered isotropically in the center-of-mass system of coordinates but not in the laboratory system of coordinates (72). Because the proton mass approximately equals the neutron mass, the res.oil angle in the laboratory system will be about half the angle in the center-of-mass system and will therefore have a range of values from zero to $\pi / 2$. However, even though the proton recoils are not scattered isotropically in the laboratory system, the membrane traversal estimates made here assume isotropic scattering in order to simplify the calculations. And, this is considered justified, since the external radiation field was in fact essentially isotropic, as the mice were rotated about the neutron source (target) and were also free to move somewhat inside the plastic capsules.

Each of the points in the equally spaced lattice can therefore be considered to have a $0.215-\mathrm{MeV}$ proton recoil emitted from it in any direction with equal probability and, since the average range is known (because average energy is known), the chance that a proton recoil from any of these points will traverse the plasma membrane can be calculated. 
The solid angle $(\Omega)$ subtended by the membrane as a function of angle, see Fig. 4.6, is given by

$$
d \Omega=2 \pi \sin \theta d \theta \text {. }
$$

Integrating from $\theta=0$ to $\theta_{2}$, we get

$$
\Omega=2 \pi\left(1-\cos \theta_{2}\right)
$$

The points $(x, y, z)$ indicated in Fig. 4.6 are chosen from the regular lattice array; angles $\theta_{2}$ and solid angles $\Omega$ are calculated using the chosen locations in space of these points, the cell radius $(6 \mu \mathrm{m})$ and the proton range $(3 \mu \mathrm{m})$. Derivation of an analytical expression to obtain the values is made here. Angle $\theta_{1}$ can be obtained using the standard triangle relationship

$$
\theta_{1}=2 \cos ^{-1}\left[\frac{P(P-R)}{r d}\right]^{1 / 2} \text {, where } P=\frac{1}{2}(r+R+d) \text {. }
$$

Then solving for $h$ (see Fig. 4.6),

$$
h=r \sin \theta_{1} \text {. }
$$


102

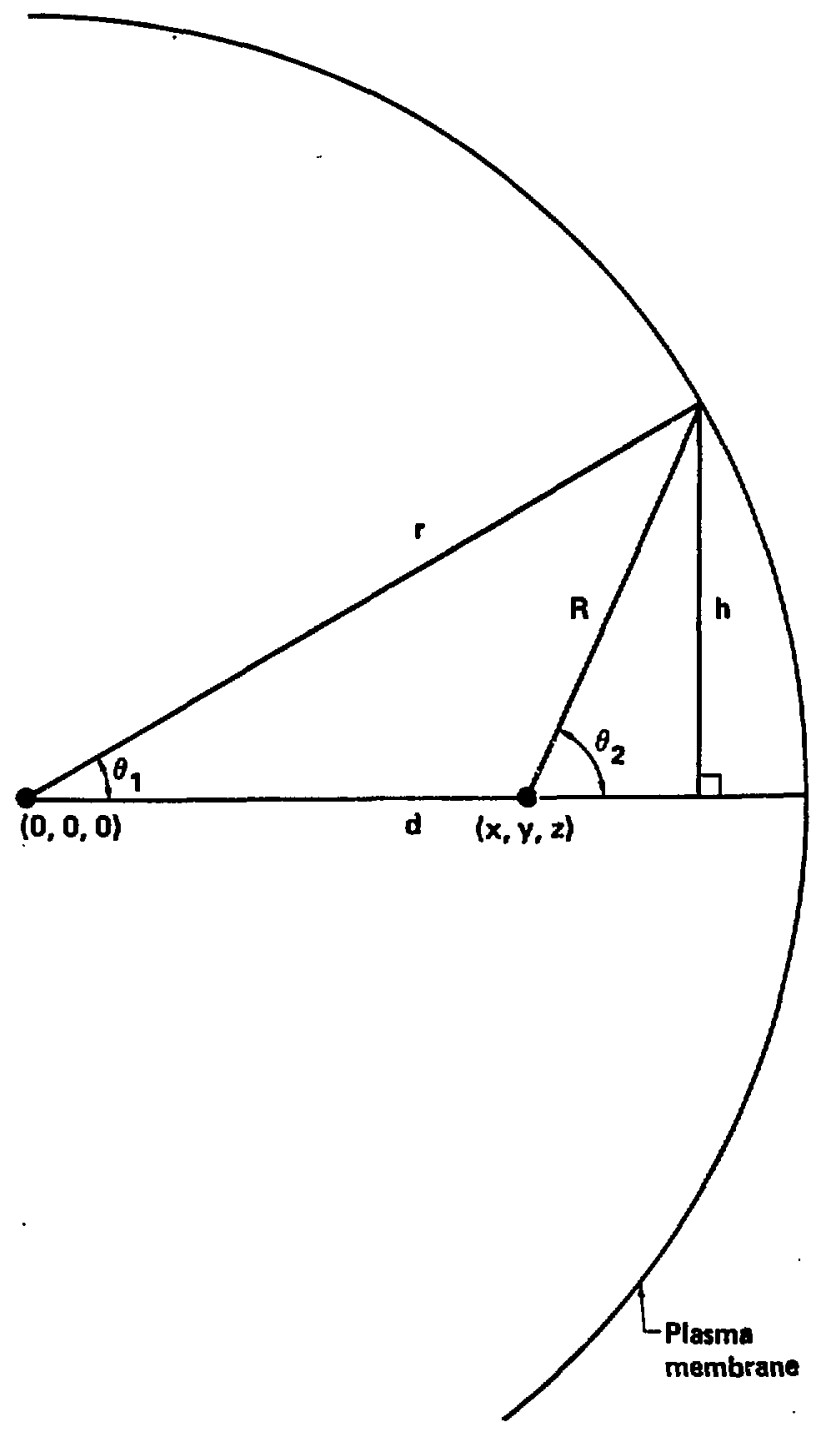

FIG. 4.6. Computational method for plasma membrane traversal by proton recoils. The center of the spherical cell (in this case, the immature mouse oocyte) has coordinates $(0,0,0)$. Point $(x, y, z)$ represents the location where a proton recoil is created by neutron interaction. The cell radius $(r)$ and the average proton recoil range (R) in water are indicated. This figure is used when $\theta_{2} \leq 90^{\circ}$. 


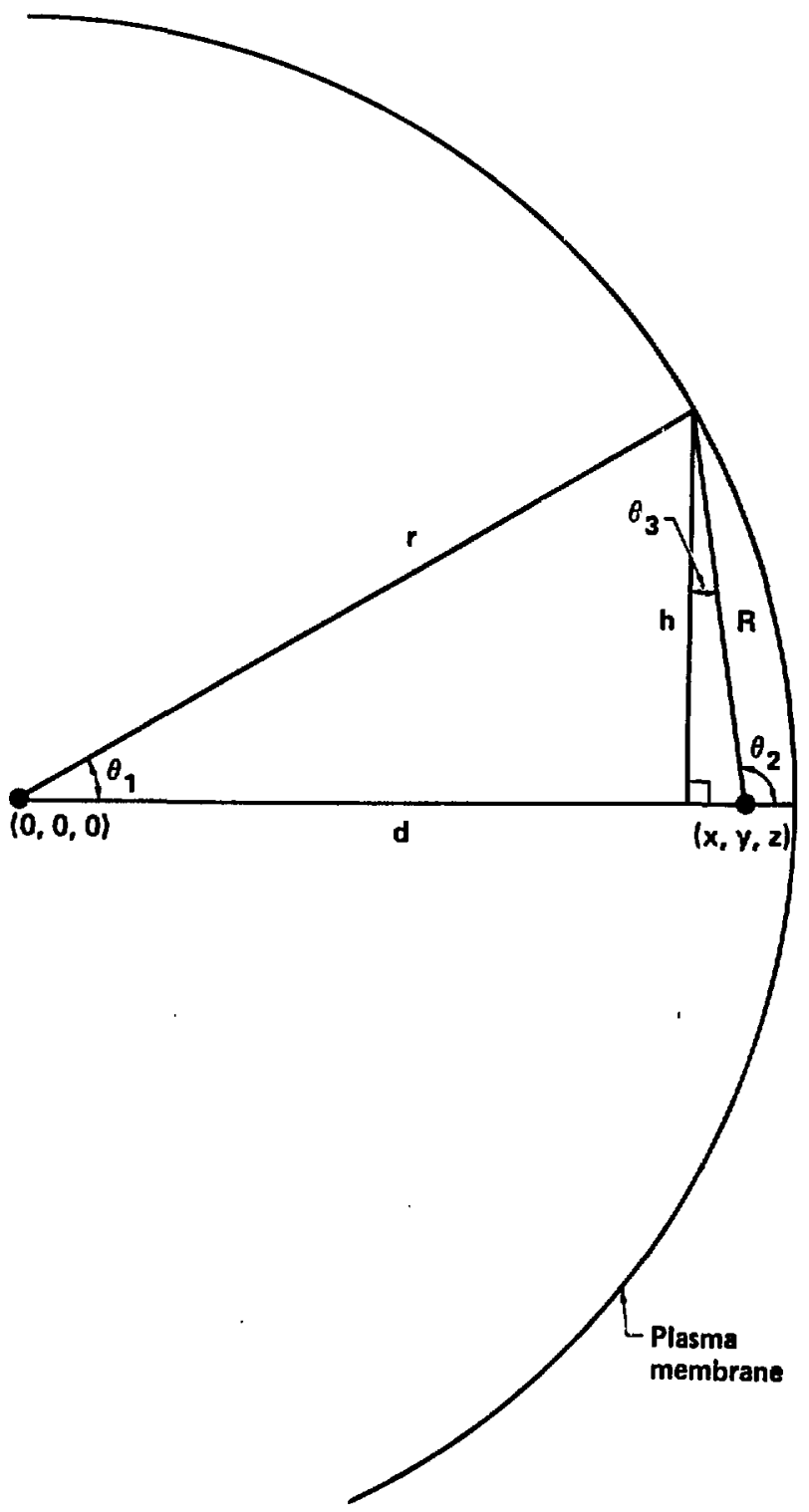

FIG. 4.7. Same aj Fig. 4.6, except here $\theta_{2}>90^{\circ}$ (see Fig. 4.6). 
$\theta_{2}\left(\leq 90^{\circ}\right)$ can now be derived using the above functions and Fig. 4.6,

$$
\sin \theta_{2}=\frac{h}{R}=\frac{r \sin \theta_{1}}{R}=\frac{r \sin \left(2 \cos ^{-1}\left[\frac{P(P-R)}{r d}\right]^{1 / 2}\right)}{R},
$$

hence

$$
\theta_{2}=\sin ^{-1}\left\{\frac{r \sin \left(2 \cos ^{-1}\left[\frac{P(-R)}{r d}\right]^{1 / 2}\right)}{R}\right\} .
$$

Substituting this $\theta_{2}$ into the solid angle equation (given above), we obtain

$$
\Omega=2 \pi\left\{1-\cos \left(\sin ^{-1}\left\{\frac{r \sin \left(2 \cos ^{-1}\left[\frac{P(P-R)}{r d}\right]^{1 / 2}\right)}{R}\right\}\right) .\right.
$$

For $\theta_{2}>90^{\circ}$, the above functions must be modified as follows:

Since

$$
\theta_{2}-90^{\circ}=\theta_{3} \text { and } \cos \theta_{3}=\frac{h}{R} \text { (see Fig. 4.7), }
$$

then

$$
\theta_{3}=\cos ^{-1}\left(\frac{h}{R}\right)=\cos ^{-1}\left\{\frac{r \sin \left(2 \cos ^{-1}\left[\frac{P(P-R)}{r d}\right]^{1 / 2}\right)}{R}\right\} .
$$


Substituting into the solid angle equation (given earlier) we obtain,

$$
\left.\Omega=2 \pi\left[1-\cos \left(\cos ^{-1} \mid \frac{r \sin \left(2 \cos ^{-1}\left[\frac{P(P-R)}{r d}\right]^{1 / 2}\right)}{R}\right\}+90^{\circ}\right)\right] \text {. }
$$

The two solid angle equations derived above are for $x, y, z$ points inside the cell only; for points ou'cside the cell, the solid angle equation becomes,

$$
\Omega=2 \pi\left[1-\cos \left(90^{\circ}-\cos ^{-1}\left\{\frac{r \sin \left(2 \cos ^{-1}\left[\frac{P(P-R)}{r d}\right]^{1 / 2}\right.}{R}\right\}\right)\right] .
$$

The $x, y, z$ coordinates along with their resuiting d's and solid angles a."e illustrated in Table 4.11 (more points were used in actual calculations). Unly $x, y, z$ points from where proton recoils are within reach of the plasma membrane are used to calculate solid angles (since only these proton recoils have greater than zeris probability of nitting the membrane). The regions both within and without the cell, from which a recoil proton may be "emitted" are illustrated in Fig. 4.8. Here $R$ is the proton recoil range and $r$. the cell radius, e.g., $R$ is $3 \mu \mathrm{m}$ for $0.43-\mathrm{MeV}$ neutrons and $r$ is $6 \mu \mathrm{m}$ for immature mouse oocytes. Regions $I$ and 4 are sufficiently distant from the plasma membrane that proton recoils created in them are unable to reach the membrane; the probability $(P)$ for plasma membrane traversal from these regions is zero. However, all locations in regions 2 and 3 are within range of the plasma membrane, and therefore proton recoils created there will have some finite (and calculable) probability of traversing the plasma membrane. Solid 
TABLE 4.11. Solid angles for plasma membrane traversal by proton recoils originating at location $x, y, z$.

\begin{tabular}{lll}
\hline $\begin{array}{c}\text { roordinates* } \\
(x, y, z)\end{array}$ & $\begin{array}{c}d \\
(\mu \mathrm{m})\end{array}$ & $\begin{array}{c}\text { smi } \\
(\mathbf{s r})\end{array}$ \\
\hline $1,1,1$ & 1.73 & $0.00 * *$ \\
$1,1,3$ & 3.32 & 1.24 \\
$1,1,5$ & 5.20 & 6.28 \\
$1,3,1$ & 3.32 & 7.24 \\
$1,3,3$ & 4.36 & 4.36 \\
$1,3,5$ & 5.02 & 7.71 \\
$1,5,1$ & 5.20 & 6.28 \\
$1,5,3$ & 5.92 & 7.71 \\
$1,5,5$ & 7.14 & 2.77 \\
$3,1,1$ & 3.32 & 1.24 \\
$3,1,3$ & 4.36 & 4.36 \\
$3,1,5$ & 5.92 & 7.71 \\
$3,3,1$ & 4.36 & 6.36 \\
$3,3,3$ & 5.20 & 3.72 \\
$3,3,5$ & 6.56 & 7.71 \\
$3,5,1$ & 5.92 & 3.72 \\
$3,5,3$ & 6.56 & 7.92 \\
$3,5,5$ & 7.68 & 5.28 \\
$5,1,1$ & 7.71 \\
$5,1,3$ & 5.20 & 2.77 \\
$5,1,5$ & 7.92 & 7.71 \\
$5,3,1$ & 3.74 & 1.92 \\
$5,3,3$ & 6.92 & 2.77 \\
$5,3,5$ & 7.92 \\
$5,5,1$ & 7.68 & 0.48 \\
$5,5,3$ & 7.14 & \\
$5,5,5$ & 7.68 & \\
\hline & 8.66 & \\
\hline
\end{tabular}

* Lattice points for $1 / 8$ sector of a mouse oocyte. This is an example, more points were used in the actual calculations.

** This coordinate is in region 1 of $\mathrm{Fig} .4 .8$ and a proton recoil created there cannot reach the plasma mc.nb:ane.

a.gles calculated from the functions derived above will now be used to obtain the probabilities for plasma nembrane traversal by recoil protons. Also, the probability $(P)$ that a recoil proton will both hit the cell and traverse the plasma membrane is calculated. 


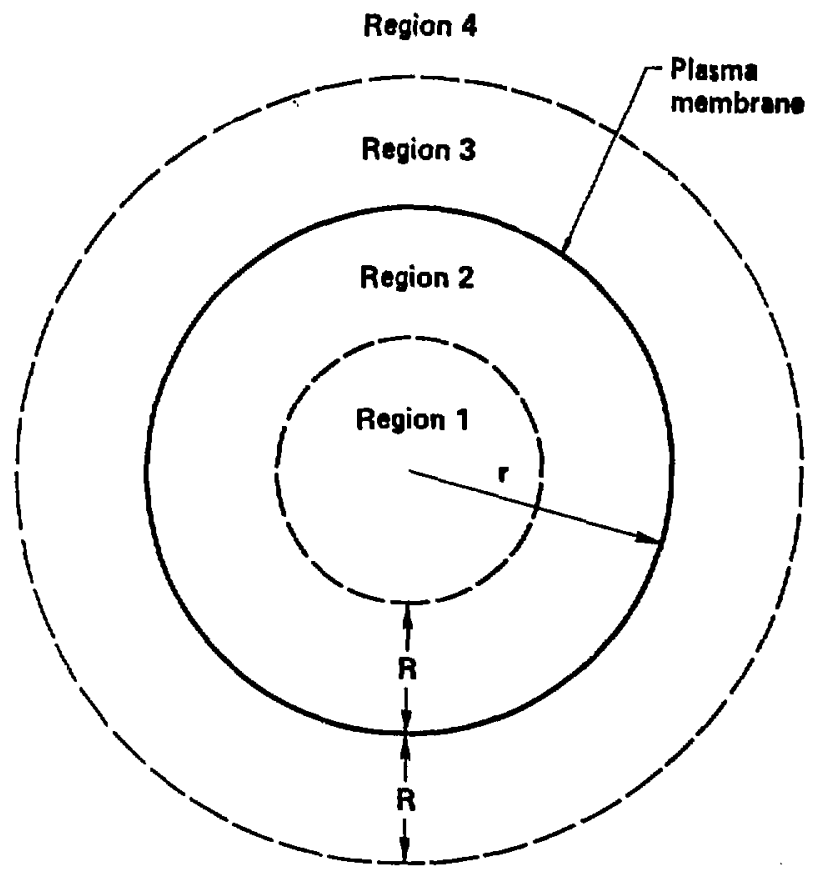

FIG. 4.8. A cross-sectional representation of an oocyte showing the regions from where a proton recoil can traverse the plasma membrane. Proton recoils created (from $0.43-\mathrm{MeV}$ neutrons) in regions 1 or 4 have zero probability of traversing the plasma membrane. However, those created in regions 2 and 3 have a finite (and calculable) probability of membrane traversal. The cell radius ( $r$ ) and proton recoil range (R) are indicated. 
Probability of membrane traversal from inside cell,

$$
P_{m i}=\frac{\Sigma \Omega_{m i}}{\Sigma 4 \pi}
$$

Probability of membrane traversal from outside cell,

$$
P_{h o}=\frac{\Sigma \Omega_{m o}}{\Sigma 4 \pi}
$$

Probability of cell hit from inside cell,

$$
P_{c i}=1 \text {. }
$$

Probability of cell hit from outside cell,

$$
P_{\text {co }}=P_{\text {mo }} \text {. }
$$

Probability of membrane traversal from ooth inside and outside cell,

$$
P_{m}=\frac{P_{m i} V_{j}+P_{m o} V_{0}}{\sqrt{i}+V_{0}},
$$

where $v_{i}$ is volume of region 2 and $v_{0}$ volume of region 3 (see Fig. 4.8).

Probability of cell hit from both inside and outside cell,

$$
P_{c}=\frac{P_{c i} V_{i}+P_{C O} V_{0}}{V_{i}+V_{0}}
$$


The ratio $P_{m} / P_{c}$, i.e., the probability that a hit in the cell will also traverse the plasma membrane, is

$$
\frac{P_{m}}{P_{c}}=\frac{P_{m i}\left(V_{i}\right)+P_{m o}\left(V_{0}\right)}{P_{c i}\left(V_{i}\right)+P_{c o}\left(V_{0}\right)} .
$$

These derived functions are general in nature and can equally well be used to calculate the probability of a recoil proton hitting the cell. nucleus while also traversing the nuclear membrane, as well as for other cell sizes and neutron energies.

Probabilitieg derived from the above functions are listed in Table 4.12 for $0.43-\mathrm{MeV}$ neutron irradiation of mouse oocytes. They show that only about $60 \%$ of the recoil protons hitting the cell also traverse the plasma membrane. Therefore, according to this model (which has been verified by exact integrational methods), the oocyte receives about 1.67 hits at the $D_{37}$, while the plasma membrane is traversed only once at this dose. The microdosimetric data obtained by Rossi $(29,57)$, shown in Fig. 4.1 for the event frequency as a function of sphere diameter, must now be modified to reflect the fact that all hits in the cell do not also traverse the plasma membrane. The event frequency at the $D_{37}$ for an exponential survival function is $1 / D_{37}$ and represents one membrane traversal at this dose. Since there are actually 1.67 hits in the cell volume per membrane traversal, the event frequency to be used with the data in Fig. 4.1 is $1.67 / \mathrm{D}_{37^{\circ}}$. With a $\mathrm{D}_{37}$ for $0.43 \mathrm{MeV}$ neutrons of $5.5 \mathrm{rad}$, the 
TABLE 4.12. Probabilities for plasma membrane traversal and cell hit by a $0.215-\mathrm{MeV}$ recoil proton*.

\begin{tabular}{lc}
\hline & Probability \\
\cline { 2 - 2 } Membrane traversal from inside cell & 0.49 \\
Membrane trayersal from outside cell & 0.12 \\
Total for membrane traversal & 0.23 \\
Hitting cell from inside & 1.00 \\
Hitting cell from outside & 0.12 \\
Total for hitting cell & 0.38 \\
Membrane traversal and cell hit & 0.60 \\
\hline
\end{tabular}

*Induced by a $0.43-$ MeV neutron.

adjusted event frequency is $0.30 \mathrm{rad}^{-1}$, which corresponds to a target diameter of about $10-12 \mu \mathrm{m}$, approximately the dianeter of the entire oocyte.

Correlating the ${ }^{3} \mathrm{H}$ results (exponential survival curve and 1 disentegration per $8 \mu \mathrm{m}^{3}$ at the $D_{37}$ ) with those for neutrons is not simple. The average number of $3_{H}$ beta rays traversing the plasma membrane at the $\mathrm{D}_{37}$ equals about 10. However, the survival curve appears exponential, indicating one lethal hit at the $\mathrm{D}_{37}$, so there seems to be a paradox. How can this be explained, and what is the effect of the remaining 9 hits? The approach used to solve this problem was, first, to determine the ${ }^{3} \mathrm{H}$ beta energy distribution 
imparted to the plasma membrane at the $D_{37}$ and, second, using this distribution of imparted energies along with calculated membrane traversal rates, to estimate the influence of the remaining 9 hits on oocyte killing. It has been established that, on average, one ${ }^{3} H$ disintegration occurs per $8 \mu^{3}$ at the $\mathrm{D}_{37}(73)$. This concentration can be represented as one ${ }^{3} \mathrm{H}$ disintegration occurring per 1.78 plasma membrane volumes, and for the purpose of this analysis, can be arranged as shown in Fig. 4.9. The volume of each assumed membrane equivalent layer does not significantly differ from that of the actual membrane since these layers are very thin, adding little to the cell diameter. Here each point represents an $3_{H}$ beta disintegration. The directional probability of the resulting beta track is considered isotropic, and therefore, as long as the disintegration occurs near the plasma membrane (within the beta range) there exists a chance that the particle will traverse it. From the model arrangement shown in Fig. 4.9 and the computational method indicated in Fig. 4.10, the probability $(P)$ of a single beta particle traversing the plasma membrane can be determined by taking the ratio of the solid angle $(\Omega)$ subtended by the membrane to the total possible silid angle $(4 \pi)$, see Fig. 4.10. The solid angle can be calculated for any given $\theta$ from the following function

$$
d \Omega=2 \pi \sin \theta d \theta \text {, }
$$




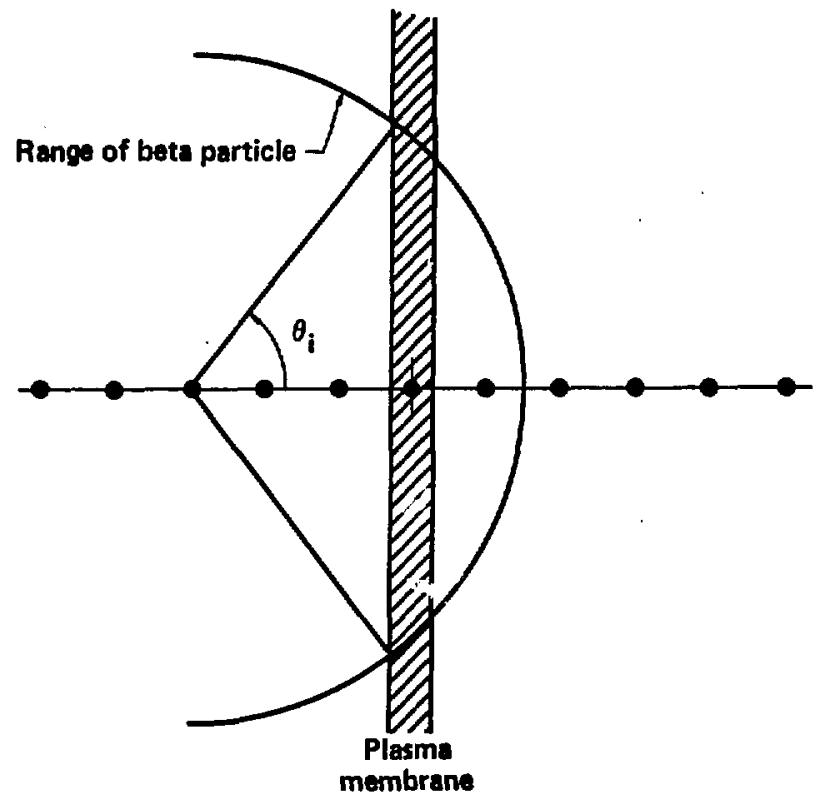

FIG. 4.9. Model arrangement of $3_{H}$ beta disintegrations near the plasma membrane (see text). Points represent $3 \mathrm{H}$ beta disintegrations and, in this mode 1, are $178 \AA$ appart at the $D_{37}$ for oocyte killing. 


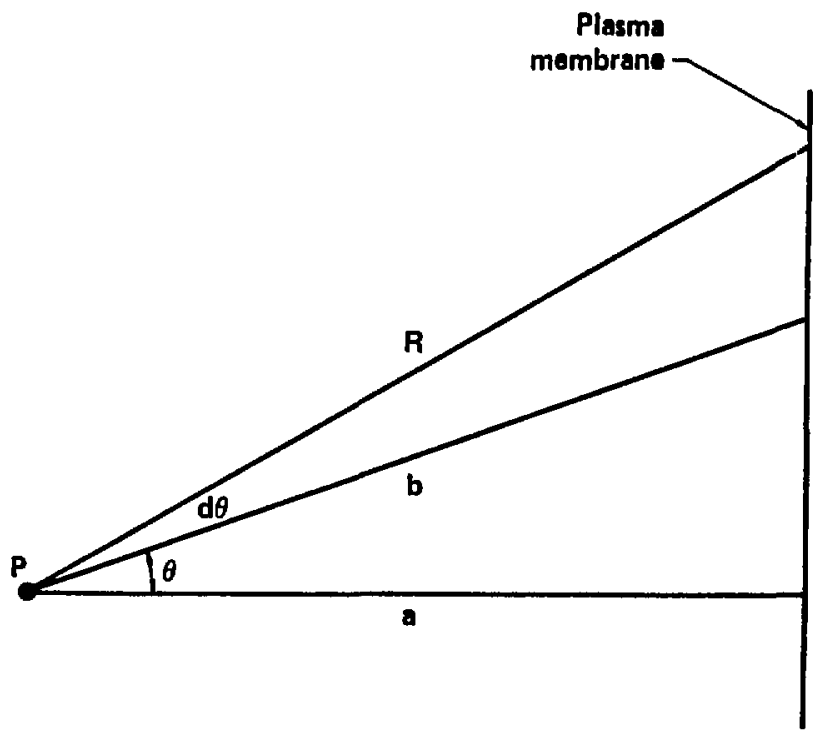

FIG. 4.10. Computational method for plasma membrane traversal by $3_{H}$ beta particle. Point (P) represents location of beta disintegration. The average beta particle range $(R)$ and the angle of traversal $\theta$ are indicated (also see Table 4.13 ). 
114

by integrating from $\theta$ to $\theta+d \theta$ we get

$$
\begin{aligned}
\Omega & =2 \pi \int_{\theta}^{\theta+d \theta} \sin \theta d \theta \\
& =\left.2 \pi(-\cos \theta)\right|_{\theta} ^{\theta+d \theta} \\
& =2 \pi[\cos \theta-\cos (\theta+d \theta)] .
\end{aligned}
$$

Results from such calculations are listed in Table 4.13 for all relevant values of $\theta$. The half-angle intervals corresponding to the selected energy intervals deposited in the plasma membrane were calculated as follows:

$$
E=\overline{\operatorname{LET}}\left[\frac{X}{\cos \theta}\right] \text {, }
$$

where $E$ is energy deposited in plasma membrane, LET is average linear. energy transfer for ${ }^{3} H$ beta rays in membrane materials and $X$ is the membrane thickness. Solving for $\theta$ we get

$$
\theta=\cos ^{-1}\left[\frac{\overline{\operatorname{LET}}(x)}{E}\right] .
$$


TABLE 4.13. Number of ${ }^{3} \mathrm{H}$ beta rays traversing plasma membrane at $\mathrm{D}_{37}$ as a function of energy deposited.

\begin{tabular}{ccccc}
\hline $\begin{array}{c}\text { Energy } \\
\text { interval } \\
(\mathrm{eV})\end{array}$ & $\begin{array}{c}\text { Half angle } \theta \\
\text { interval } \\
\text { (deg) }\end{array}$ & $\begin{array}{c}\Omega \\
(\mathrm{sr})\end{array}$ & $\mathrm{P}$ & $N$ \\
\hline $100-199$ & $0-60$ & 3.10 & 0.25 & 8.4 \\
$200-299$ & $60-70.5$ & 1.00 & 0.08 & 0.9 \\
$300-399$ & $70.5-75.5$ & 0.50 & 0.04 & 0.2 \\
$400-499$ & $75.5-78.4$ & 0.31 & 0.025 & 0.08 \\
$500-699$ & $78.4-81.8$ & 0.37 & 0.03 & 0.12 \\
$700-999$ & $81.8-84.3$ & 0.27 & 0.02 & 0.06 \\
$1000-6000$ & $84.3-90$ & 0.62 & 0.05 & 0.34 \\
\hline
\end{tabular}

The total number of plasma membrane traversals in each energy deposition interval has been calculated and is given in Table 4.13. The computational functions derived for this particular problem are shown below. The number $(N)$ of ${ }^{3} H$ beta particles traversing the plasma membrane within any angular interval is given by the function

$$
N=2 P \frac{R[\cos \theta-\cos (\theta+d \theta)]}{D} \text {, }
$$

where $R$ is the beta particle range, $D$ the membrane thickness, and

$$
P=\frac{\Omega}{4 \pi}=\frac{(\cos \theta-\cos (\theta+d \theta)}{2}
$$


For $R=0.6 \mu \mathrm{m}$ and $D=0.0178 \mu \mathrm{m}$ (see Fig. 4.10 ),

$$
N=67.4 P[\cos \theta-\cos (\theta+d \theta)] .
$$

The probability (P) of membrane traversal for any given increment of angle (calculated from the above function) is also given in Table 4.13. The total number of membrane traversals (for all angles. combined) at the $D_{37}$ is then obtained by summing the numbers in the various intervals (see Table 4.13) and equals about 10. However, only one of these traversals deposits $300 \mathrm{eV}$ or more at the $D_{37}$, indicating that some $300 \mathrm{eV}$ or more must be imparted to the plasma membrane by an ${ }^{3}$ beta particle to result in oocyte killing. This is only about half the energy required by neutrons (600 eV) and suggest that ${ }^{3} \mathrm{H}$ beta rays are particularly efficient in the killing of immature oocytes.

Figure 4.11 shows the resulting frequency distribution of beta-particle membrane traversals as a function of energy deposited. It is interesting to note the substantial skewing of the frequency distribution to lower energy intervals, i.e., about $83 \%$ of the hits deposit less than $200 \mathrm{eV}$ in the membrane and most of these are closer to $100 \mathrm{eV}$ and may therefore be similar in effectiveness to ${ }^{60} \mathrm{Co}$ gamma-rays. Since a single ${ }^{3} \mathrm{H}$ beta ray depositing at least $300 \mathrm{eV}$ to the plasma membrane appears to be sufficient for cell inactivation, the 9 beta rays delivering less than this (most deliver 100-200 eV, and at a very low dose rate) can reasonably be expected to have effects similar to those of ${ }^{60}$ Co gama rays given chronicaliy. The oocyte survival curve for low dose rate ${ }^{3} \mathrm{H}$ beta 


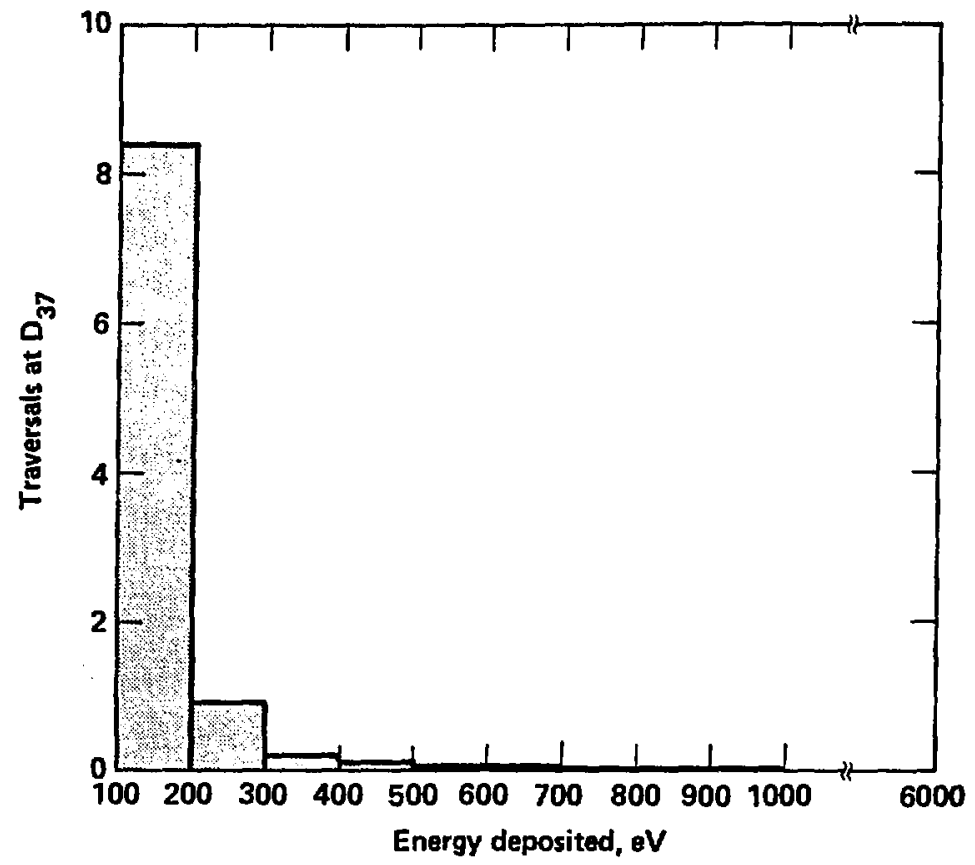

FIG. 4.11. The number of $3 H$ beta particles traversing plasma membrane as a function of average deposited energy. The number of traversals is evaluated at the $D_{37}$ for oocyte kiling in juvenile mice as determined by (73). 
rays obtained by Dobson and Kwan (41) may therefore be made up of two components: one similar to chronic ${ }^{60}$ Co gamma rays, yielding a sigmoidal survival curve, and another, more like higher LET radiation, giving an exponential survival curve. Using the fitted linear-quadratic dose-response function for chronic ${ }^{60} \mathrm{Co}$ (from Reference 41) and an exponential function with $\mathrm{D}_{37}$ of $8.5 \mathrm{rad}$ for $3_{H}$, the two components were separated and the results listed in Table 4.14. The survival curve for $E \geq 300 \mathrm{eV}$ is taken to be exponential (since only one plasma membrane traversal at this energy deposition level appears to kill the oocyte), whereas the function for $E<300 \mathrm{eV}$ is simply taken as equal to that for chronic ${ }^{60} \mathrm{Co}_{0}$ gamma rays. The analytical expression reconstructed from these two components is

$$
S(x)=100 \exp =\left(5.7 \times 10^{-2}\right) 0+\left(5.72 \times 10^{-2}\right) 0+\left(5.84 \times 10^{-4}\right) 0^{2} .
$$

Although it is not exponential, the departure is not noticable at 24 rad (maximum dose used by Dobson and Kwan (41). It may, in fact, not be significantly different from an exponential function even at doses causing total oocyte depletion ( $240 \mathrm{rad})$, see Table 4.14. If ${ }^{3} H$ beta rays are given at higher dose rates, however, the lower energy deposition events may produce a larger quadratic coefficient (as is the case for ${ }^{60} \mathrm{Co}$ ) thereby causing the ${ }^{3} \mathrm{H}$ survival curve to noticably depart from that of an exponential function. It is postulated here, therefore, that the ${ }^{3} \mathrm{H}$ dose-response curve (41) appears exponontial only because the ${ }^{3} H$ betas are delivered at very low dose rate, and should the dose rate 
TABLE 4.14. Effect of $3^{3}$ beta ray exposure on mouse oocytes.

\begin{tabular}{llccc}
\hline \multicolumn{5}{c}{ Oocyte survival $(\boldsymbol{x})$} \\
$\begin{array}{ccccc}\text { Dose* } \\
\text { (iad) }\end{array}$ & $\begin{array}{c}\text { Observed } \\
\text { total }\end{array}$ & $\begin{array}{c}\text { Separated } \\
(E \geq 300 \mathrm{eV})\end{array}$ & $\begin{array}{c}\text { Separated } \\
(E<300 \mathrm{eV})\end{array}$ & $\begin{array}{c}\text { Expected } \\
\text { total }\end{array}$ \\
\hline & & & & \\
10 & 30.7 & 57 & 53 & 30.1 \\
20 & 9.4 & 32 & 25 & 8.1 \\
30 & $2.9 * *$ & 18 & 10.6 & 1.9 \\
40 & $0.9 * *$ & 10 & 4.0 & 0.4 \\
\hline
\end{tabular}

* Effective dose.

** Extrapolated from data (41) with maximum sose used of 24 rad. t From derived analytical expression given above.

be substantially increased, the model presented here would predict a slightly sigmoldal survival curve. As previously noted, the vast majority of energy absorption events from ${ }^{2}$ il beta rays in the membrane are similar in magnitude to those from ${ }^{60}$ Co gamma rays and can therefore be expected to produce similar effects. The dose-response data for low-dose-rate ${ }^{60}$ Co gamma rays (41) indicate that at doses below about 10 rad the survival curve is well fitted by an exponentia? function, and in fact, even at 20 rad only slight departure is observed. Below these doses both high-energy and low-energy deposition events are expected to yield nearly exponential survival curves at the dose- dies used by Dobson and Kwan (41). However, at larger dose rates some departure from an exponential function is expected; an attempt should be made to verify this through further experiments. 


\section{CONCLUSIONS}

Available dose-response data for oocytes show inconsistencies that are here reconciled by considering the plasma membrane as the sensitive target. It appears that about $500 \mathrm{eV}$ (somewhat less for $3_{H}$ beta rays and slightly more for $0.43-\mathrm{MeV}$ neutrons) delivered acutely to the plasma membrane are required for cell destruction. Data in support of the plasma membrane as the sensitive target in these cells are summarized in Table 4.15. They are strongly suggestive.

An interesting observation is that the target appears to change with the animal's age. In 14-day-old mice the plasma membrane is implicated, whereas, in the fetal animal two days before birth ( 17 days postconception) the target appears to be the cell nucleus. Such striking variations in the same experimental system have not previously been reported and may have further radiobiological implications, e.g., possibly large differences in neutron-induced mutation frequencies depending on age at exposure. 
TABLE 4.15. Evidence for plasma membrane as the radio sensitive target in mouse oocytes.

observation

$3_{H}$ confined to the mouse oocyte nucleus produces mych (vastly) less cell killing than does ${ }^{H}$ generally distributed

Microscopic studies show correlation between early cell volume changes and radiation exposure of young oocytes

For $0.43-\mathrm{MeV}$ neutrons, target cross-sectional area is larger than nucleus and about the size of the entire cell

For ${ }^{60} \mathrm{Co}$ gamma rays, target volume about equals volume of oocyte plasma membrane

The energy requirements for cell killing are similar for all radiation types studied when plasma membrane is considered as target

The neutron RBE for oocyte killing is exceptionally low, which has also been observed for other endpoints where membrane damage is implicated
Reference

Inferred from data given in References 41 and 66

Straume (this paper)

11

II

This paper and reference 56 


\section{A SYNTHESIS OF THE HUMAN AND ANIMAL MEUTRON RBE DATA}

\section{HUMAN NEUTRON RBE'S}

The Japanese A-bomb experience is the only human in vivo neutron exposure data involving sufficient numbers of exposed persons to allow determination of neutron dose-response curves for a variety of late effects. The data collected from A-bomb survivors are therefore extremely important to human radioblology*.

The bomb radiation in Hiroshima and Nagasaki, made up of both neutrons and gamma rays, had differing neutron dose components in the two cities. Neutrons were a significant feature at Hiroshima but not at Nagasaki, and differences in dose-response curves found in the two cities for a number of endpoints are usually ascribed to differences in the neutron dose components. Assuming this to be the case, the data can be analyzed to provide neutron dose-response curves and gamma-ray dose-response curves, using the method first described by Rossi and Mays (75) and modified slightiy by Straume and Dobson (Appendix 1 and 2). This method is illustrated in Table 2 of Appendix 2 for chromosome aberrations in blood lymphocytes of A-bomb survivors. Derived Hiroshima neutron effects can be plotted against

\footnotetext{
* An impressive effort has been underway since 1950 by many organizations in the collection and analys is of data, most notably by the Radiation Effects Research Foundation (formerly the Atomic Bomb Casualty Comission) and to some degree the UN and NAS. The data interpretations by these scientific bodies are used by the radiation protection community (e.g., ICRP and NCRP) to estimate human health risks from ionizing radiation.
} 
corresponding neutron doses, and the data points fitted by linear regression, thereby providing dose-response curves for neutrons. Assuming equal effectiveness for neutrons in both cities, pure gamma-ray dose-response curves can be obtained by subtracting out any effect of the very small amount of neutrons at Nagasaki. The neutron RBE can then be obtained from the fitted dose-response functions by using the formula described on Page 55, and as shown in Fig. 4 of Appendix 2. The RBE is seen to increase with decreasing dose and to reach high values at small toses.

Accurate knowledge of the true doses received by the A-bomb survivors is essential for reliable RBE evaluation. Recently, the doses from the Hiroshima and Nagasaki bombings have been re-evaluated $(76,77)$, and the new estimates are considerably different from the T65 doses previously widely accepted. The T65 doses referred to as T65D (for "tentative 1965 doses") were determined by Auxier, et al. who initially published dose-versus-range functions for each city (78). These functions were then used by others to assign doses to the A-bomb survivors (79). If these doses are incorrect (which now appears likely), earlier biomedical interpretations on the effectiveness of neutrons in man may be in error. This question has been examined in some detail for leukemia, breast cancer, total malignancies, and chromosome aberrations (see Appendixes 1, 2 and 3).

For many effects, both $T 65$ and re-evaluated doses give neutron RBE values that increase with decreasing dose and reach high values as dose diminishes. However, because of the smaller difference between dose-response curves for Hiroshima and Nagasaki with the new 
dosimetry, some effects (acute leukemia and breast cancer) now yield RBEs that are not significantly different from unity. And for some endpoints the data are insufficient to clearly reveal the shapes of dose-response curves and therefore can not be used in a Rossi-Mays-type RBE analysis. Such data are fitted here by simple linear regressions for those malignancies showing in the Life Span Study sample (80) significant correlation with radiation exposure. Ratios of the slopes of these linear regressions using the new dose estimates for Hiroshima (SH) and for Nagasaki (SN), are listed in Table 5.1. They are approximately proportfonal (but by no means equal) to the RBE for Hiroshima neutrons. An approximate relation to estimate the neutron RBEs for all effects listed in Table 5.1 can be obtained as follows. Two effects with contrasting RBEs, the values of which can be established with the Rossi-Mays method (e.g., breast cancer and total malignancies), are used as "known" data points. A straight line passirig through these points is equivalent to the analytical function, $\mathrm{RBE}=m(\mathrm{SH} / \mathrm{SN})+b$, where $m$ is the slope of the line and $b$ the $y$-axis intercept. The function derived for Loewe-Mendelsohn dose estimates is: $\mathrm{RBE}=84.3 \times(\mathrm{SH} / \mathrm{SN})-82$, and that for "RR" doses (described below) is: RBE $=38.6 \times(\mathrm{SH} / \mathrm{SN}$ ) - 38. Rough estimates of RBE can then be obtained for any value of (1) SH/SN. Neutron RBES estimated in this way are included in Table 5.2, together with RBE values (for endpoints having reliable doseresponse curves) obtained directly by the Rossi-Mays method. 
TABLE 5.1 Ratios of carcinogenic efficiency for Hiroshima and Nagasaki radiation (using new dose estimates).

Slope of Hiroshima curve

Malignancy

Slope of Nagasaki curve

Leukemia (all)

1.1

All malignancies

2.4

Esophagus

high*

Stomach

1.3

Digestive tract

2.3

Trachea, bronchus, and lung

3.0

Female breast

1.0

Urinary organs

1.1

Lymphatic and

hemopoietic system

(except leukemia)

0.8

- Specific values can not be obtained since slope of Nagasaki curve is negative, however high RBE is clearly indicated.

Neutron RBE values determined with "radiobiologically reconciled" (RR) doses (see Appendix 3) are also included in Table 5.2. It is noted that RBE values obtained with RR doses are a factor of 2 to 3 lower (at the same neutron dose) than those derived with the Loewe-Mendelsohn doses, and show improved agreement with experimental results (see Fig. 9 in Appendix 3 ).

Probably the most striking observation from Table 5.2 is the large differences in neutron RBE for different endpoints. Although 
TABLE 5.2. Neutron RBE estimates for human radiation effects.

Neutron RBE* at 1 rad

with LM** doses with "RR" doses

EFFECTS WITH LOW RBE

\begin{tabular}{|c|c|c|c|}
\hline Total leukemia (all forms) & 1 & {$\left[\begin{array}{ll}11\end{array}\right]$} & 1 \\
\hline Acute leukemia & 1 & {$\left[\begin{array}{ll}1 & 1\end{array}\right]$} & 1 \\
\hline Lymphatic cancer (except leukemia) & & {$\left[\begin{array}{ll}1 & 1\end{array}\right]$} & \\
\hline Breast cancer & 1 & {$\left[\begin{array}{ll}1 & 1\end{array}\right]$} & 1 \\
\hline Urinary cancer & & {$[11]$} & \\
\hline Stomach cancer & & & \\
\hline
\end{tabular}

EFFECTS WITH HIGH RBE

Total malignancies (all forms)

$119[119]$

$55[55]$

Chronic granulocytic leukemia

$163[178]$

$70[81]$

Digestive tract cancer

Esophageal cancer

[112]

high $^{\dagger}$

[51]

[171]

high ${ }^{\dagger}$

Trachea, bronchus, and lung cancer

$111[109] \quad 47[50]$

[78]

Chromosome aberrations

- RBE values not in brackets are obtained directly by the Rossi-Mays method. Values in brackets are estimated by the functions given on p.122.

** Loewe-Mende 1sohn doses.

$+\mathrm{SH} / \mathrm{SH}$ (and therefore RBE) could not be determined since SN was negative. However, high values are clear iy indicated.

such variations should not be surprising, as they have been clearly demonstrated in animals (see Fig. 2.5), the attempt to quantify and 
classify these RBES and to compare them to those derived from the animal data has not previously been undertaken.

It is of interest to compare the neutron RBEs for various malignancies to the ease with which these malignancies are induced by low-LET radiation. Since the effectiveness of low-LET radiation is strongly influenced by intracellular recovery mechanisms, one would expect the RBE to be low for malignancies that are readily induced by low-LET radiation (low recovery), whereas larger values would be expected when low-LET radiation is less efficient (high recovery). Table 5.3 lists the relative sensitivities to radiation induction of breast cancer, lung cancer, leukemia, and gastrointestinal cancer versus the relative neutron RBEs for each. The above expectations are indeed observed, and therefore lend support to these RBE estimates.

TABLE 5.3. Human sensitivity to cancer induction by low-LET radiation and neutron RBE compared.

\begin{tabular}{lll}
\hline Malignancy & $\begin{array}{l}\text { Relative* } \\
\text { sensitivity }\end{array}$ & RBE \\
\hline Female breast & High & Low \\
Lung & Moderate & High \\
Leukemia & Very high & Low \\
Gastrointestinal & Low to moderate & High \\
\hline
\end{tabular}

* From reference 3. 
Experimental neutron RBEs were discussed in Chapter 2 and will now be compared with those determined for humans. Table 5.4 classiffes neutron RBEs obtained for various endpoints from both animal and human data. Although important similarities exist, differences are observed for such effects as breast cancer and

TABLE 5.4. Animal and human neutron RBE data compared.

\begin{tabular}{|c|c|c|}
\hline & $\begin{array}{l}\text { Animal } \\
\text { data }\end{array}$ & $\begin{array}{l}\text { Human } \\
\text { data }\end{array}$ \\
\hline \multicolumn{3}{|l|}{ EFFECTS WITH LOW RBE } \\
\hline Total Leukemia (a11 forms) & see specific & Appendix 1 \\
\hline Acute leukemia (a)l forms) & types & Appendix 3 \\
\hline Acute granulocytic leukemia & Rf mice (28) & $\star$ \\
\hline Thymic leukemia & RF mice (26) & $\star$ \\
\hline $\begin{array}{l}\text { Lymphat ic cancer } \\
\text { (except leukemia) }\end{array}$ & RF mice (26) & see Table 5.2 \\
\hline Breast tunors & $\mathrm{BALB} / \mathrm{c}$ mice $(25)$ & Appendix 1 \\
\hline Ovarian tumors & BALB/C, RF mice $(25,26)$ & see Table 5.2 \\
\hline
\end{tabular}

EFFECTS WITH HIGH RBE

Acute granulocytic leukemia

CBA mice (96)

Chronic granulocytic leukemia $\|$

Appendix 3

Lung tunors

Breast tumors

Chromosome aberration
BALB/C, RF mice $(25,27)$ see Table 5.2 RF mice (26)

Sprague-Dawley rat (6)

(C3H $\times$ 101) mice (35) Appendix 1

* Neutron RBE data not available. 
reticular malignancies and illustrate the difficulty in simple extrapolation from animal data to man.

It is interesting that several of the effects yielding low neutron RBEs in both animals and man (see Table 5.4) are known or suspected of being associated with virus $(81,83)$. This is generally not true of those with high RBE values. In fact in one high-RBE case, chronic granulocytic leukemia in man, a different association, namely with chromosomal abnormality (the Philadelphia chromosome) is clearly established (82). It may therefore be of etiological significance that when neutron and gamma-ray dose-response curves for chromosome aberrations are superimposed on those for mouse mamary tumors close correspondence is found for RFM mice, whereas BALB/C mice (noted for virus-related mammary tumors (81)) compare poorly. The human-to-animal intercomparison for each effect can be summarized as follows.

\section{Leukemia}

In man, the induction of acute leukemia appears to be associated with low neutron RBEs and, as it makes up most of radiation-induced leukemia, total leukemia also has low RBE. On the other hand, high neutron RBEs are found for human chronic granulocytic leukemia, a disease having known association with chromosome abnormality. In mice also, anywhere from low to high neutron RBE values are observed for leukemia, and are functions of both leukemia type and mouse strain. There is no clear evidence that chromosome abnormalities (such as the Philadelphia chromosome in humans) are associated with 
leukemia in animals, although it has been shown in several species and strains that viruses are invoived (83).

\section{Lymphoma}

These malignancies appear to yield low neutron RBEs in man, similar to those observed for acute leukeinia. LOW RBE values are also obtained in animals (mice), where viruses are implicated (83).

\section{Lung Cancer}

In both animals and man neutron RBEs are high for lung cancer induction, which may reflect similarities in mechanisms of carcinogenic action.

\section{Female Breast Tumors}

Animal data on breast cancer indicate high neutron RBEs for some species and strains and lower values for others (Table 5.4). It is interesting to note that marmary tumor induction in Sprague-Dawley rats (6) and BALB/C mice (25) with low-LET radiation yield approximately linear dose-effect curves, which are not greatly influenced by dose-rate (30). However, Sprague-Dawley rats give large neutron RBEs while BALB/C mice give low values. This peculiarity (with the'rat system) is discussed in Chapter 2. In man, lOW-LET dose-effect curves are fitted equally well with linear or linear-quadratic functions (see Appendix 1). Neutron RBEs for human breast cancer are not significantly different from unity (see Appendix 1). 
All Malignancies Combined

The neutron RBE for mortality from all malignancies combined should reflect the collective RBE for those malignancies that result in death. These values are therefore expected to vary for different species in relation to the types of malignancies most frequentiy induced in them by radiacion. For example, in RFM mice leukemias and lymphomas are the main neoplasms induced and they have low neutron RBEs, while in Sprague-Dawley rats mammary tumors (some were not malignant) are induced and show large RBEs. In man, the organs contributing most to cancer mortality from neutron irradiation are the lungs and gastrointestinal tract, both with high neutron RBEs (see Appendix 1 and Reference 80). These malignancies, therefore, result in high neutron RBE values for mortality from all (combined) malignancies (see Fig. 5 in Appendix 1).

\section{Chromosome Aberrations}

In all biological materials (including human) studied to date with both neutrons and gamma rays, high RBEs have been observed for chromosome aberrations (e.g., References 10,11,32,35, and Appendixes 1, 2 , and 3). The general nuture of this phenomenon probabiy reflects the common physical character of the genetic material and its organizational structure, which is remarkably similar in all eukaryotic cells (except for differences in numbers of chromosomes). Interspecies variations in the relative effectiveness of neutron and gamma radiations are therefore expected to be small, as indeed is observed (see Fig. 2.7). 
Specific Locus Mutations

The vast majority of biological materials exhibit neutron RBEs for specific-locus mutacions that increase with decreasing dose and are similar to those obtained for chromosome aberrations, e.g., somatic cel1 mutations in Tradescantia stamen hairs $(4,5)$, somatic mutations in the leaves of maize $(33,34)$, specific-locus and dominant mutations in mouse spermatogonia (36-39), and mutations in mature mouse oocytes $(30,39)$. However, mutations in immature mouse oocytes, cells which are peculiarly sensitive to radiation killing, have not been demonstrated $(30,39)$. It is suggested here that for such mutations to be observable with gamma rays, smaller doses than are usually used in mutation studies would have to be used, less than 50 rad, which would necessitate using very large numbers of animals. Neutron irradiation, on the other hand, in the energy region between a few hundred keV and one or two MeV may be very inefficient for inducing mutations in the immature mouse oocyte (at least during the highly sensitive juvenile stage) since it appears that a single membrane traversal by this radiation results in oocyte death (see Chapter 4).

Radiation-induced heritable mutations have not been clearly demonstrated in man (84); the Japanese A-bomb data are suggestive, but too imprecise to yield values that are significantly different from zero. However, the vast body of data from animals (e.g., 4,5, $30,33,34,36-39)$, indicate that high neutron RBEs are the rule for mutagenesis and that a possible exception (which needs further study) may be the highly radiosensitive immature mouse oocyte. The human 
immature socyte does not appear to be especialiy radiose sitive $(30,39,85)$ and may therefore behave more like the mature oocytes in the mouse, for which the neutron $\operatorname{RBE}$ is high $(30,39)$.

IMPL ICATIONS FOR RADIOLOGICAL HEALTH PROTECTION

Since the amount of biological demage caused by a given dose of radiation is usually a function of the radiation's LET, a dose-modifying factor has been adopted for radiological health protection purposes. A value For neutrons of this Quality Factor (Q) was chosen $(86,87)$ from the neutron RBE literature of the 1950 s and 1960s, which indicated that a value of 10 for fast neutrons was sufficiently conservative to accommodate even the highest RBES suspected at that time for human effects (86). However, more recent reports shoving increasing neutron RBE with decreasing dose, reaching (for some expe. "mental endpoints) ...ues well over 100 at very low doses, have stimulated a move to re-evaluate the presently accepted neutron safety standards. The 1978 report (75) that leukemia data from the Japanese A-bomb survivors provided neutror, RBEs in good agreement with numerous observations made in experimental systems, reaching values of about 60 at one rad (with no signs of leveling off at lower doses), appears to have prompted the NCRP to issue a statement (88) indicating their intention to review the neutroi: $Q$ presently in use, and that an upward revision of this value may 
be forthcoming. Arguments have been made on both sides of this question $(89,90)$ and the issue remains unresolved.

Developments during the past year, however, have made the above observation for human leukemia obsolete. The re-evaluated Hiroshima and Nagasaki doses (76) remove what appeared (with T65 dosimetry) to be a clear inter-city difference for total leukemia, and suggest instead that the neutron RBE for this endpoint in man is close to nity (76 arf Appendix 1). Inter-city differences are, however, observed with the new doses for a number of other endpoints, and these do allow neutron RBE calculations (Appendixes 1,2, and 3).

Neutron RBEs derived from the A-bomb data are highly uncertain at this time, reflecting the prelininary nature of the new dose estimatas. Uncertainty in RBE is expected to decrease when dose values become firmly established. However, there will always be substantial uncertainty, even if the doses were known precisely. A rough estimate can be made of the minimum uncertainty attainable in neutron RBE estimation from the Japanese data, reflecting uncertainties in both biuiogical effect ard ausorbed dose. The uncertainty in biological data is a function of the nuinber of cases per dose group and is not expected (at least for malignancies) to change appreciably with time. The uncertainty in the dose is made up of many individual factors, e.g., weapon yield, radiation output per unit of yield, radiation transport to each survivor and, more specifically, to each relevant tissue or organ. Some of these dosimetric parameters are expected to be improved upon (through work presently being undertaken at several locations (91)), but as will be 
shown here, any likely improvenent in the accuracy of these parameter values is not expected to appreciably affect the overall uncertainty in neutron RBE derived from them.

Table 5.10 (page 144) lists estimates of minimum attainable uncertainties* in physical and biological parameters, and compares them to values presently quoted. It can be inferred froil Table 5.10 that statistical uncertainties in the biomedical data alone (at least $\pm 50 \%$, and unlikely to be substantially improved) make up about half of the total uncertainty in the dose-effect relationships. Such large uncertainties in the dose-effect parameters, and consequently in RBE determination, 1 imit the usefulness of the Japanese A-bomb data (by themselves) in giving specific guidance for a neutron quality factor for radiological health protectior. The A-bomb data must be used in conjunction with animal data and theoretical considerations.

The experimental neutron RBE data shown in Figs. 2.4, 2.5, 2.7, 2.10, and 2.11 impressively illustrate that, except for various effects in dry plant seeds, the RBE does not substantially exceed the furction $500_{n}^{-1 / 2}$. This is true irrespective of species or endpoint and should perhaps be considered a general "law" of radiobiology. A number of effects, however, give lower RBEs, including some human malignancies, as shown in Fig. 2.5 and Table 5.2. But other human effects do have neutron RBEs consistent with $500_{n}^{-1 / 2}$. This function may be considered conservative

* These values were obtained as indicated in Table 5.10. 
for estimating the neutron RBE of effects where it can not be excluded.

Dose limits for radiological health protection purposes are established on the basis of an acceptable level of risk to the exposed individual (or population). Since various radiations have differing effectiveness (produce different levels of risk at the same dose), a dose-normalizing factor (Q) has been established by ICRU (87) so that all radiations produce equal levels of risk per unit of "normalized dose." Using recent data to obtain the most appropriate value for $Q$, neutron risk coefficients (risk per unit dose) are derived for the most important human health effects. These coefficients can be obtained by several methods, all with inherent uncertainties. Four such methods are discussed and compared below.

\section{Method 1. Rossi-Mays-Type Analysis}

This method, described in Appendix 2, results in considerabie uncertainty (as do the other methods) when all coefficients of variation are propagated through the required arithmetic steps (as done in Appendix 1). It is, however, the only method that uses exclusively human data to obtain a neutron dose-response curve and is therefore the most direct analysis available. The estimatea lifetime neutror $r_{r}$ isk coefficient for total malignancies obtained with this method, using the new re-evaluated dosimetry, is $114 \times 10^{-4}$ per person rad. In Table 5.8 (page 141) it is compared to values estimated by other methods and appears somewhat high. 
The inherent uncertainties $(U)$ in the Rossi-Mays method can be estimated from the following function:

$$
u=\left(u_{\text {effect }}^{2}+u_{\text {dose }}^{2}\right)^{1 / 2}
$$

The uncertainty in biological effect is about $\pm 58 \%$ (50\% Nagasaki and 30\% Hiroshima) at the dose leveis of importance here. Dose uncertainties can be estimated by considering all individual dosimetric parameters that enter into the Rossi-Mays-type analysis (see Table 5.10).

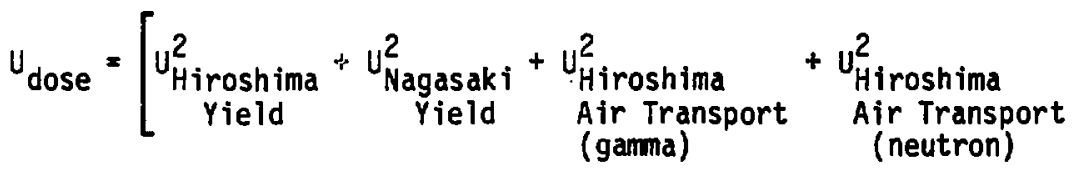

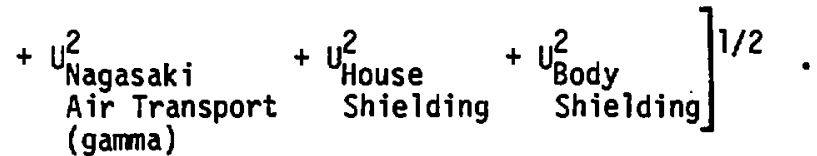

Inserting values from Table 5.10

$$
u_{\text {dose }}=\left[20^{2}+10^{2}+20^{2}+20^{2}+20^{2}+10^{2}+20^{2}\right]^{1 / 2}=47 \% \text {. }
$$

Hence the total uncertainty becomes

$$
U=\left[58 \%^{2}+47 \%^{2}\right]^{1 / 2}=75 \% \text {, and is compared in }
$$

Table 5.10 to those derived for the other methods. 
Method 2. Modified Rossi-Mays-Type Analysis

This is a slight variation of Method 1. Since the re-evaluated dosimetry $(76,77)$ appears to have underestimated neutrons* for Hiroshima by a factor of about five (see Appendix 3), the neutron risk-coefficient can be reduced by this factor, resulting in a value of $22 \times 10^{-4}$ per person rad, which is consistent with estimates obtained by other methods (Table 5.8).

\section{Method 3. Neutron Risk Coefficients Estimated}

\section{from Low-LET Dose-Response Data at Low Doses}

The low-LET risk coefficients obtained from fitted dose-response functions at low doses can be used together with estimated neutron RBEs to derive neutron risk coefficients. Tabie 5.5 lists these values for leukemia, breast cancer, and all other cancers combined. The Japanese A-bomb data indicate neutron RBEs for leukemia (total leukemia) and breast cancer not different from 1. The RBE for all other cancers, however, appears much larger and is taken here isee page 133 for rationale) to be 50 at 1 rad of neutrons. An effective neutron RBE for all malignancies combined can then be determined from the data presented in Table 5.5 by weighting the RBE for each effect by its Low-LET risk coefficient. Calculating the effective neutron RBE, we obtain

$$
R B E=\frac{50(1.2)+1(0.2)+1(0.8)}{1.2+0.2+0.8}=28 \text {. }
$$

* Other explanations are possible (e.g., gross underestimation of Hiroshima yield or systematic problems in the biomedical data) but are much more difficult to reconcile. 
TABLE 5.5. Risk-coefficients derived by Method 3.

\begin{tabular}{ccc} 
& Low-LET & Neutron \\
risk & RBE \\
Cffect & coefficient* & at 1 rad \\
\hline
\end{tabular}

Leukemia

$\begin{array}{ll}0.8 & 1 \\ 0.2 & 1 \\ 1.2+ & 50\end{array}$

Breast cancer**

All other cancers

50

* Risk coefficients are deaths $/ 10^{6}$ person year rad.

** Estimated mortality from incilence data (given in Appendix 1), using mortality/incidence ratios in Reference 3 and a male to female ratio of 0.5 .

+ Value for mean plus 2 SD is used since the mean is negative at low doses (see Table 5.6). This will result in an upper-limit estimate for the neutron risk-coefficient.

For a 30-year rish period* the total lifetime neutron risk-coefficient $\left(R_{n}\right)$ becomes

$$
\begin{aligned}
R_{n} & =\left(2.2 \times 10^{-6} \text { per person year rad }\right) \times(28) \times(30 \text { years }) \\
& =18.5 \times 10^{-4} \text { per person rad. }
\end{aligned}
$$

The uncertainty $(U)$ in this value can be estimated by

$$
U=\left[U_{\text {effect }}^{2}+U_{R B E}^{2}\right]^{1 / 2}
$$

* The generally assumed time interval of excess cancer mortality risk from a radiation exposure (92). 
where $U_{\text {effect }}$ is the uncertainty in biological effect (Poisson distribution of number of cases) and is roughiy $300 \%$ at low doses (10 rad) (see Appendix 1). The uncertainty in neutron RBE can be approximated by the range between low and high values for leukemia and breast cancer. The maximum effective RBE is expected if leukemia and breast cancer both have values of 25 at low doses (maximum RBE consistent with dosewresponse curves at $+1 S D$ ) and therefore,

$$
R B E_{\max }=\frac{50(1.2)+25(0.2)+25(0.8)}{1.2+0.2+0.8}=39 .
$$

The uncertainty in RBE can be roughly estimated as

$$
U_{R B E}=\left(\frac{39-28^{*}}{28}\right) 100=39 \%
$$

The total uncertainty in the neutron risk-coefficient becomes

$U=\left[2300 x^{2}+39 x^{2}\right]^{1 / 2}=303 \%$

This value is included in Table 5.8 .

* This value is the effective neutron RBE derived on page 136 and is considered nominal. 
Method 4. Neutron Risk Coefficients Estimated

from Low-LET Dose-Response Data at High Doses

This involves the same procedure used in Method 3, with the exception that low-LET data are taken at high doses, thereby minimizing the possibly error-producing effect that any particular curve-fitting model might have on the results. A major uncertainty here involves use of "dose-magnitude-effectiveness factors" from animal data to reduce the low-LET human risk coefficients observed at high doses to values that could be expected at low doses (it should be pointed out however that such factors derived from the human (Nagasaki) data are similar to those derived here from animals for leukemia and breast cancer; factors for total màlignancies are similar only when derived at the mean +2 SD (also see Table 5.5)). Table 5.7 summarizes relevant data. When the estimated low-dose risk coefficients are compared to coefficients derived for low doses directly from the human data (see Table 5.6), good agreement is seen for leukemia and breast cancer. The risk coefficient for total malignancies, however, being inconsistent with those for leukemia and breast cancer at low doses when determined directly from the Nagasaki curve (see Table 5.6), now becomes compatible with these data. Again an effective neutron RBE is derived (as in Method 3 ),

$$
R B E=\frac{50(1.6)+1(0.2)+1(1)}{1.6+0.2+1}=29 \text {, }
$$

which gives a lifetime neutron risk coefficient for total malignancies of

$$
\begin{aligned}
R_{n} & =\left(2.84 \times 10^{-6} \text { per person year rad }\right) \times(29) \times(30 \text { years }) \\
& =24 \times 10^{-4} \text { per person rad. }
\end{aligned}
$$


TABLE 5.6. LOW-LET risk coefficients at low doses ( $10 \mathrm{rad}$ ) estimated directly from linear-quadratic curve fitting.

Effect

Risk coefficient*

Leukemia

0.80

Breast cancer

0.27

Total malignancies

$-0.70$

* Deaths $/ 10^{6}$ person year rad.

TABLE 5.7. LOW-LET risk coefficients at low doses estimated from high doses $(200 \mathrm{rad})$.

\begin{tabular}{|c|c|c|c|}
\hline Effect & $\begin{array}{l}\text { High-dose } \\
\text { risk } \\
\text { coefficient* }\end{array}$ & $\begin{array}{c}\text { Dose } \\
\text { magnitude } \\
\text { effectiveness } \\
\text { factor } \star \star\end{array}$ & $\begin{array}{c}\text { Est imated } \\
\text { low dose } \\
\text { risk } \\
\text { coefficient* }\end{array}$ \\
\hline Leukemia & 4.8 & 0.2 & 1.0 \\
\hline Breast cancert & 0.6 & 0.3 & 0.2 \\
\hline Total malignancies & 5.8 & 0.5 & 2.8 \\
\hline
\end{tabular}

* Deaths $/ 10^{6}$ person year rad.

* Obtained from Reference 30 using animal data.

+ Estimated as in Table 5.5. 
TABLE 5.8, Neutrci risk coefficients for total malignancies derived by the four methods.

Method

Risk-coefficient*

\begin{tabular}{lc}
1 & $114 \pm 75 \%$ \\
2 & $22 \pm 75 \%$ \\
3 & $18.5 \pm 303 \%$ \\
4 & $24 \pm 66 \%$ \\
\hline
\end{tabular}

* Deaths per $10^{4}$ person rad.

The uncertainty $(U)$ in this value can be estimated as follows:

$$
U=\left[U_{E f f e c t}^{2}+U_{R B E}^{2}+U_{\text {Animal data }}^{2}\right]^{1 / 2} .
$$

The uncertainty in effect at high doses is about $\pm 50 \%$. The uncertainty in RBE can be estimated from limiting values, e.g. the RBE at a neutron dose of 1 rad can range from 1 to 25 for leukemia and breast cancer and is probably not more than 50 for all other malignancies combined. This range of possible neutron RBEs results in about $30 \%$ variation in the effective RBE. Uncertainty in the animal data can be estimated from the variation between different data sets used to derive the dose-magnitude-effectiveness factors listed in Table 5.7, and equals $37 \%$. Total uncertainty in the neutron risk-coefficient then becomes

$$
U=\left[50 x^{2}+30 x^{2}+31 x^{2}\right]^{1 / 2}=66 \%
$$


In Table 5.8 this value is compared with those obtained by the other methods.

The lifetime neutron risk coefficients given in Table 5.8 for each method can be compared with the level of risk considered acceptable by ICRP (1.25 per $10^{4}$ person rad) for low-LET radiation (92). An estimate of $Q$ can be obtained from the ratio,

$$
Q=\frac{\text { derived neutron risk coefficient }}{\text { acceptable low-LET risk coefficient }} \text {. }
$$

$Q$ values estimated in this way are given in Table 5.9 for each method along with their minimum* ranges of uncertainty. The most probable value for $Q$ can be taken as the average of the values obtained by Methods 2, 3, and 4 (Method 1 is excluded for reasons discussed in Appendix 3) and equals 17 . This is very different from the value obtained by Method i $(Q=91)$ villich deniands further explanation.

From the preceding analyses it is clear that the uncertaitity in the derived $Q$ value is substantial. In fact, the best possible estimate will have an associated uncertainty of almost $\pm 100 \%$ : Considering the uncertainties in these estimates of $Q$ and the fact that the most probable value derived here of 17 is not very different

Minimun uncertainties reflect the "minimum uncertainty" values given in Table 5.10. These values are estimates of the smallest uncertainties attainable during the forseeable future. Present uncertainties are also indicated in Table 5.10 and are larger. 
TABLE 5.9. Neutron "quality factor" estimates derived by the four methods.

\begin{tabular}{llc}
\hline Method & $\begin{array}{c}\text { "Quality* } \\
\text { factor" }\end{array}$ & $\begin{array}{c}\text { Minimum } \\
\text { range of } \\
\text { values }\end{array}$ \\
\hline 1 & 91 & $23-159$ \\
3 & 18 & $5-32$ \\
4 & 14 & $1-42$ \\
\hline
\end{tabular}

* Taken as the ratio of the neutron risk coefficient (derived here by each method) and the accepted gamma-ray risk coefficient given by ICRP (92).

from the value of 10 presently in use, it is questionable whether a revision of the neutron Quality Factor is justified at this time. It must be added, however, that if the data (both physical and biomedical) used in Method 1 are shown to be substantially correct, and if the difference between Hiroshima and Nagasaki dose-response curves result from the larger neutron component in Hiroshima (rather than from, e.g., differences in the two populations not previously accounted for) then an upward revision of $Q$ must be seriously considered. It is therefore important at this time, while the dosinetry is being re-evaluated, to further understand the biomedical data, especially data independent from Hiroshima/Nagasaki. 
TABLE 5.10. Estimated uncertainties* in Hiroshima and Nagasaki dosimetry and biological effects data.

\begin{tabular}{|c|c|c|c|c|}
\hline Data & $\begin{array}{c}\text { Present } \\
\text { uncertainty } \\
(\%)\end{array}$ & Reference & $\begin{array}{l}\text { Minimumk* } \\
\text { uncertainty } \\
(x)\end{array}$ & Ref: 'ence \\
\hline \multicolumn{5}{|l|}{ Source term } \\
\hline Hiroshima & $>20$ & 76,77 & 20 & $t$ \\
\hline Nagasaki & $>10$ & 76,77 & 10 & + \\
\hline \multicolumn{5}{|l|}{ Air transport } \\
\hline \multicolumn{5}{|l|}{ Hiroshima } \\
\hline Neutrons & $50+t$ & 76 & 20 & 93 \\
\hline Gamma rays & 30 & 76 & 20 & 93 \\
\hline \multicolumn{5}{|l|}{ Nagasaki } \\
\hline Neutrons & 50 & 76 & 20 & 93 \\
\hline Garma rays & 30 & 76 & 20 & 93 \\
\hline
\end{tabular}

\section{Shielding}

Houses

Body

Biomedical effects

Large doses

20

$>20$

Small doses

50
$>50$

Appendix 1

50

Appendix 1
10

93

b

$\geq 20$

b

* Coefficients of variation.

** See text for explanation.

t These values are for weapon yields alone and are not expected to be substantially decreased.

t+ The uncertainty in Hiroshima neutron dose may be substantially larger than this (see Appendix 3).

a Inferred from preliminary estimates by Jess Marcum, R\&D Associates, May 1981.

b This uncertainty in dose can easily result from uncertainties in the directional positioning of survivors; the value $+20 \%$ is estimated for bone marrow. 


\section{SUMMARY AND CONCLUSIONS}

FACTORS AFFECTING NEUTRON RBE

Many factors are observed to influence tive neutron RBE. They include the level of dose at which the KBE is determined, energy of neutror radiation, energy and dose rat.: of the comparison radiation, and the biological material and effect being studied. For higher energy neutrons, i.e., above a few MeV, dose rate of neutron radiation may also influence $R B E$. Though all of these factors may affect RBE, they do not affect it equally. For example, changes in neutron dose may alter the RBE by facturs of $50 \pm 0100$, whereas variations in neutron energy will typically alter the RBE by factors of only 2 to 3 . The strong influence that dose can exert on neutron RBE does not usually result from dose-related changes in neutron effectiveness, but rather from the well known decrease in effectiveness of low-LET radiation, the comparison radiation ( $x$ or gamma rays), with decreasing dose. This decreased effectiveness of low-1.ET radiation is thought to result from a diminished probability that the products of ionization will interact between particle tracks. The observed changes in RBE with radiation energy, however, result from changes in the LET of the secondary particles, and therefore are believed to reflect changes in the probability that the products of ionization will interact within tracks. Since these two factors (dose and energy) affect the neutron RBE by different mechanismi, it is not surprising that they should also influence it to different degres. 
In general, the neutron RBE increases with decreasing dose. The relationship often follows the expression $R B E=k D_{n}^{-1 / 2}$, where $k$ is an empirical constant and $D_{n}$ is the neutron dose. Many biological endpoints, however, do not have neutron RBE relationships that follow this function (or in some cases, only partially). Cell killing sometimes falls into this category, as does somatic mutation induction in Tradescancia at very low doses. Interestingly, cataract induction in mice appears to follow this neutron RBE relationship for one mouse strain ( 7 ) but not for another (8). This suggests inter-strain variabilities at the level of the sensitive target. Certain malignancies, in toth animals and man, also do not appear to follow this neutron RBE function, e.g., induction of mouse ovarian tumors, induction of mammary tumors in RFM mice, and the induction of breast cancer and of acute leukemia in humans. These effects do not show neutron RBEs significantly greater than unity, even at the lowest doses tested (in some cases only a few rad of neutrons).

A peculiarly high neutron RBE is observed for certain effects in plants following irradiation of dry seeds. These RBEs, which may reach values in excess of 1000 at low doses, are drastically reduced to values compatible with those for wet animal tissues when seeds are soaked in water prior to irradiation. This can be explained by the relatively grater importance of indirect radiation action (damage caused by radiation hydrolys is products formed in the water medium) $\because$ ith gamma rays than with neutrons.

The neutron RBE for life shortening simply reflects the collective RBEs of the individual induced effects that result in 
mortality. For acute modes of death, the values reflect the RBEs of the target cell populations, while for lethality from late affects, the values reflect the RBEs of the malignancies that contribute most to the animal mortality.

It is concluded here that although many factors (physical, chemical, and biological) can influence the neutron RBE, they do not influence it by the same amount, nor do they appear to affect it by the same underlying mechanisms. Ranking these factors according to their ability to affect the neutron RBE (see Table 6.1) demonstrates that neutron dose, tissue water content, and biological endpoint are most important, whereas neutron energy, $\mathrm{O}_{2}$ concentration, and neutron dose rate are of lesser importance.

\section{EFFECT OF NEUTRONS ON MOUSE OOCYTES}

Experiments on immature mouse oocytes with neutrons and garma rays have shown that neutron RBEs for this highly radiosensitive endpoint (radiation-induced oocyte killing in the 14-day-0ld mouse) are conspicuously low; in fact, the highest value attained (at 0.1 rad of $0.43 \mathrm{MeV}$ neutrons) was less than 2! This is interesting since this neutron RBE does not follow the general trend observed in several low-dose studies on other endpoints (Tradescantia stamen hair mutations, rat mammary tumors, and mouse cataracts) and may reflect a contrastingly different radiosensitive target. It is argued here that this target is probably the oocyte's plasma membrane. Supportive evidence includes target size estimates, observed 
TABLE 6.1. A ranking of factors according to their ability to affect neutron RBE.

\begin{tabular}{|c|c|}
\hline Factor & Rank* \\
\hline Neutron dose & 1 \\
\hline $\begin{array}{l}\text { Water content of biological } \\
\text { material during irradiation }\end{array}$ & 2 \\
\hline Biological endpoint & 3 \\
\hline Dose-rate of comparison radiation & 4 \\
\hline Energy of compartson radtation & 5 \\
\hline Energy of neutrons & 6 \\
\hline $\begin{array}{l}\text { Molecular oxygen concentration in } \\
\text { biological material during irradiation }\end{array}$ & 7 \\
\hline Dose-rate of neutrons & 8 \\
\hline
\end{tabular}

* Number 1 is most effective;

consistency in the energy deposited for all radiations tested $\left({ }^{3} \mathrm{H}\right.$ beta rays, ${ }^{60}$ Co gamma rays, $0.43-\mathrm{MeV}$ neutrons, fission neutrons and $15-M e V$ neutrons), and previous data indicating that ${ }^{3} \mathrm{H}$-thymidine incorporated into DNA is very much less effective in immature oocyte cell killing than is ${ }^{3} \mathrm{HOH}$ distributed throughout the cell.

The induction of genetic effects in mature mouse oocytes yields neutron RBEs consistent with the general trend observed for other endpoints whereas for immature oocytes in the "resting" phase of ganetic development an RBE has, up to now, not been demonstrated. In fact, genetic effects have not been observed with either gamma rays or neutrons in these cells. It is suggested here that, with $x$ or gamma rays, all of the immature resting oocytes in the mouse will be 
killed at doses large enough for genetic effects to be induced in detectable amounts. It is also suggested that with neutrons of moderate energy ( $0.4 \mathrm{MeV}$ to $2 \mathrm{MeV}$ ), an observable genetic effect may be difficult to obtain from immature mouse oocytes. The reason for this is that these oocytes appear to be killed by single proton recoils traversing the plasma membrane; hence, genetic damage without simultaneous killing of the cell can result only from those proton recoils that traverse the nucleus but not the plasma membrane. The probability for this is very low for neutrons of moderate energy. When mice are irradiated just prior to birth their oocytes are much less radiosensitive to killing than at 14 days of age, and neutron RBEs consistent with most other endpoints are observed. The sensitive target size at that time is somewhat smaller than the oocyte nucleus. Similar results are suggested for mice irradiated on the 11 th postconception day (about I week prior to birth), although the gamma-ray data are less than adequate for this age period, limiting our confidence in RBE values.

ine following conclusions are drawn regarding the effects of neutrons on mouse oocytes:

- The effectiveness of neutrons in killing mouse oocytes during their most radiosensitive stage in development is not very much different from that of ${ }^{3} \mathrm{H}$ beta rays or ${ }^{60}$ Co gamma rays. Maximum RBE values are less than 2. This unusual RBE response (at least for cell killing) can be explained by considering the plasma membrane as the radiosensitive target. 
- During less radiosensitive stages (e.g., just prior to birth) oocyte killing appears to require the nucleus to be hit.

- The neutron RBE increases with decreasing dose for the killing of immature oocytes, and becomes constant at very low dose. It is important to note that this response, expected from the theory of dual radiation action, is observed in what is here believed to be a non-DNA target.

- Californium-252 fission neutrons are less effective in the killing of immature oocytes than are acute ${ }^{60}$ Co gamma rays. This also can be explained by considering the plasma membrane as the radiosensitive target.

- Neutron RBE for immature oocyte killing is strongly influenced by the mouse's age at exposure. The change in RBE with age appears to result from a change from one radiosensitive target to another, viz., from the cell nucleus at 17 days postconception to the plasma membrane by 14 days postpartum. Hormonal influences have been suggested in these changes; however, no definitive indications of this have been found.

\section{EFFECTIVENESS OF NEUTRONS IN MAN}

Neutron effect data in man are obtainable from survivors of the atomic bombings in Hiroshima and Nagasaki, a few nuclear oriticality accident cases, neutron radiotherapy patients, and in vitro studies on cultured human cells. Each of these data sources have certain aspects that make the assessment of neutron effects difficult, e.g., 
the survivors of Hiroshima and Nagasaki (also criticality accident victims) received primarily gamma rays, making estimates of pure neutron effects uncertain. Cancer pacients receiving neutron therapy are exposed to only partial body doses, and because of the existing malignancy may also have received chemotherapy, making data analys is difficult. The most valuable data for neutron-effect analyses are those from Hiroshima and Nagasaki, from criticality accidents (where. good dosimetry has been made), and from chromosome-aberration studies on human lymphocytes irradiated in vitro (similar responses are seen in vitro and in vivo). Neutron RBEs have been calculated from each of these data sets and are summarized in Table 6.2 (see also Appendices 1 and 3).

Neutron RBEs for other human effects, those where adequate dose-response curves for Hiroshima and Nagasaki could not be derived but for which induction by radiation can be established, were obtained by fitting linear regressions to the data for each city and then using the resulting regression values to estimate RBEs (see Table $5.1, p .123)$. The neutron RBEs estimated in this way were then compared to those obtained from animal data are found. Important similarities and interesting differences are found. For example, chromosome aberrations and cancer of the lung are associated with large neutron RBEs in both animals and man, whereas, RBEs are low for breast cancer in man, but anywhere from low to high in animals. Such contrasting RBE values for malignancies of the same organ in various species reflect specific differences that can be identified. By studying these differences, basic carcinogenic mechanisms may be 
TABLE 6.2. Summary of neutron RBEs for hunan effects.

Source of neutrons

Effect

RBE

(at 1 rad neutrons)

Hiroshima*

Acute leukemia

Breast Cancer

Chronic granulocytic leukemia

Total malignancies

70

Chromosomal exchanges

55

47

$Y-12$ accident

Chromosomal exchanges

49

Experimental

Chromosomal exchanges

(fission neutrons)

(human, in-yitro)

* The "RR" doses described in Appendix 3 were used here.

uncovered. The similarly low RBEs observed for acute leukemia and breast cancer in humans, and for mammary tumors and leukemia in some mice suggest the possibility of fundamental similarities at the target level. Since some radiation-induced leukemias and marmary tumors in mice are known to be associated with viruses $(83,81)$, it is hypothesized here that such associations may also be present in human breast cancer and human acute leukemia. A possible mechanism involved could be radiation-induced DNA single strand breaks that allow viral genome insertion into host DNA. Since neutrons are not much more effective in causing single strand breaks than are gama rays, a low neutron RBE would be expected. 
The human data are inadequate for determining neutron RBE values with the precision necessary to establisn standards for radiological health protection. Similarly, the animal data are of insufficient accuracy (and unceriain human relevance) to allow direct use for this task. Fundamental assumptions are therefore required in translating derived neutron RBE values into radiological protection standards. These assumptions should take account of all data sources (human, animal, and theoretical) and the inkerent uncertainties in each. Methods that oraw from each of these data sources have been usied here to estimate an appropriate neutron quality factor (Q) for radiological health protection. "Q" values estimated by four different methods are given in Table 5.9 (p. 143) along with their minimum possible uncertainties. The uncertainty in these values are substantial, and clearly demonstrate the fact that neutron-effect values (e.g., risk coefficients and RBES, and therefore quality-factor estimates) are unlikely to become more accurately known than this regardless of how much effort is brought to bear on the problem! This is important to emphasize. It plays an essential role in the controversial neutron RBE debate, and has not been adequately considered in the published literature. Although the above discussion paints a rather pessimistic picture for the establishment of accurate neutron radiological protection standards, our confidence can be increased by comparing values derived by independent methods and from data sources. Results from these independent methods do show a reasonable degree of similarity. They indicate a best estimate for $Q$ of about 17 and a possible range of values (at \pm ISD) from 1 to 30 . 
Valuable information regarding fundamental mechanisms of radiation carcinogenesis may be obtained by comparing neutron RBES from human data to those for experimental animals (in which mechanisms are better understood).

And it is concluded that the most appropriate value for the neutron quality factor (estimated here as 17 ) is in reasonable agreement with that presently accepted. Considering the uncertainty . in this derived value $( \pm 100 \%)$, revision of the presently accepted neutron quality factor, which has a value of 10 , does not appear indicated at this tine; at least not while the Hiroshima/Nagasaki doses are under re-evaluation. 


\section{REFERENCES}

1. Rossi, H.; "Comments on the Somatic Effects Section of the BEIR III Report," Radiat. Res. 84, 395-406 (1980).

2. Kellerer, A. M. and Rossi, H. H.; "The Theory of Dual Radiation Action," Curr. Top. Radiat. Res. Q. 8, 85-158 (1972).

3. National Research Council, Advisory Committee on the Biological Effects of Ionizing Radiations, "The Effects on Populations of Exposure to Low Levels of Ionizing Radiation," Nat'. Acad. Sci., Washington, D.C., (T980).

4. Sparrow, A. H. and Rossi, H. H.; Mutations Induced in Tradescantia by Small Doses of X-Rays and Neutrons: Analysis of Dose-Response Curves," Science 176, 916-920 (1972).

5. Underbrink, A. G. and Sparrow, A. H.; "The Inf luence of Experimental Endpoint, Dose, Dose-Rate, Neutron Energy, Nitrogen Ions, Hypoxia, Chromosome Volume and Ploidy Level on RBE in Tradescantia Stamen Hairs and Pollen," in: Biological Effects of Neutron Irradiation, IAEA, Vienna (1974), Pp. 185-2/4.

6. Shellabarger, C. J., Kellerer, A. M., Rossi, H. H., Goodman, L. J., Brown, R. D., Mills, R. E., Rao, A. R., Shanley, J. P., and Bond, V. P.; "Rat Mammary Carcinogenes is Following Neutron or X-Radiation," in: Biological Effects of Neutron Iradiation, IAEA, Vienna (1974) pp. 391-401.

7. Bateman, J. L., Rossi, H. H., Kellerer, A. M., Robinson, C. V., and Bond, V. P.; "Dose-Dependence of Fast Neutrons RBE for Lens Opacification in Mice," Radiat. Res. 51, 381-390 (1972).

8. Di Paola, M. and Coppola, M.; "Biological Responses to Various Neutron Energies from 1 to $600 \mathrm{MeV}$ : Lens Opacification in Mice," Radiat. Res. 84, 453-461 (1980).

9. Straume, T.; "A Comparative Radiation Study Using the Transplantable C $3 \mathrm{HBA}$ Mammery Adenocarcinoma: The RBE of cyclotron Neutrons," M. S. thesis, University of Washington, Seattle, WA (1976).

10. Lloyd, D. C., Purrott, R. J., Dolphin, G. W., Bolten, D., and Edwards, A. A., and Corp, M. J.; "The Relationship Between Chromosome Aberrations and LOW LET Radiation Dose to Human Lymphocytes," Int. J. Radiat. Biol. 28, 75-90 (1975).

11. Lloyd, D. C., Purrott, R. J., Dolphin, G. W., Bolten, D., and Edwards, A. A.; "Chronosome Aberrations Induced in Human Lymphocytes by Neutron Irradiation," Int. J. Radiat. Biol. 29, $169-182$ (1976). 
12. Hall, E. J., Novak, J. K., Kellerer, A. M. Rossi, H. H., Marino, S., and Goodman, L. J.; "RBE as a Function of Neutron Energy. I. Experimental Observations," Radiat. Res. 64, 245-255 (1975).

13. Kellerer, A. M., Hall, E. J., Rossi, H. H., and Teedla, P.; "RBE as a Function of Neutron Energy," II. Statistical Analys is," Radiat. Res. 65, 172-186 (1976).

14. Berry, R. J., Mpodification of Neutron Effects Upon Cells by Prepair, and by Physical and Chemical Means;" in: Biological Effects of Neutron Irradiation, IAEA, Vienna (1974), pp. 257-27/.

15. Broerse, J. J., Barendsen, G. W. and Van Kersen, G. R.; "Survival of Cultured Human Cells after Irradiation with Fast Neutrons of Different Energies in Hypoxic and Oxygerrated Conditions," Int. J. Radiat. Biol. 13,559-572 (1968).

16. Broerse, J. J. and Barendsen, G. W.; "Relative Biological Effectiveness of Fast Neutrons for Effects on Norma 1 Tissues," Curr. Top. Radiat. Res. Q. 8, 305-350 (1973).

17. Hall, E. J.; "RBE and OER Values as a Function of Neutron Energy," Europ. J. Cancer 10, 297-299 (1974).

18. Field, S. B. and Hornsey, S., "RBE Values for Cyclotron Neutrons for Effects on Normal Tissues and Tumors as a Function of Dose and Dose Fractionation," Europ. J. Cancer 7, 16 1-169 (1971).

19. Bateman, J. L., Johnson, H. A., Bond, V. P., Rossi, H. H.; "The Dependence of RBE on the Energy of Fast Neutrons for Spermatogonia Depletion in Mice," Radiat. Res. 35, 86-101 (1968).

20. Coggle, J. E., Gordon, M. Y., Lindop, P. J., Shewe II, J., and Mil1, A. J.; "Some In Vivo Effects of $\pi$-Mesons in Mice," Br. J. Radiol. 49, 161-165 (1976).

21. Di Paola, M., Caffarelli, V., Coppola, Porro, F., and Quintiliani, $M$; "Biological Responses to Various Neutron Energies from 1 to $600 \mathrm{MeV}$. I. Testes Weight Loss in Mice," Radiat. Res. 84, 444-452 (1980).

22. Conger, B. Y. and Constantin, M. J.: "The Effectiveness of Fission Neutrons, 14.7-MeV Monoenerget ic Neutrons and $60_{0}$ Gamma Radiatiolr on Seedling Growth Reduction and Induction of Chlorophyll-Deficient Mutations in Barley," in: Biological Effects of Neutron Irradiation, IAEA, Vienna (1974), pp. 4T7-432. 
23. Lu, C. C., Meistrick, M. L., and Thames Jr., H. O.; "Survival of Mouse Testicular Stem Cells after $\gamma$ or Neutron Irradiation," Radiat. Res. 81, 402-415 (1980).

24. Phillips, T. L. and Fu, K. K.; "Biological Effects of $15 \mathrm{MeV}$ Neutrons," Int. J. Radiat. Onc. Biol. Phys. 1, 1139-1147 (1976).

25. Ullrich, R. L., Jernigan, M. C. and Storer, J. B.; "Neutron Carcinogenes is Dose and Dose-Rate Effects in BALB/C Mice," Radiat. Res. 72, pp. 487-498 (1977).

26. Ullrich, R. L., Jernigan, M. C., Cosgrove, G. E., Satterfield, L. C., Bowles, N. D., and Storer, J. B.; "The Influence of Dose and Dose Rate on the Incidence of Neoplastic Disease in RFM Mice After Neutron Irradiation, "Radiat. Res. $68,115-131$ (1976).

27. Ullrich, R. L.; "Effects of Split Doses of X-rays or Neutrons on Lung Tumor Formation in RFM Mice," Radiat. Res. 83, 183-145 (1980).

28. Upton, A. C., Randolph, M. L. and Darden, E. B. Jr.; "Relative Biological Effectiveness of Fast Neutrons for Late Somatic Effects in Mice," in: Biological Effects of Neutron and Proton Irradiation, IAEA, pp. 337-345 (1964).

29. Rossi, H. H.; "The Role of Microdosimetry in Radiobiology," Radiat. Environ. Biophys. 17, 29-40 (1979).

30. National Council on Radiation Protection and Measurements, "Influence of Dose and its Distribution in Time on Dose-Response Relationships for Low-LET Radiations," NCRP Report 64, (1980).

31. Lloyd, D. C., Purrott, R. J., Reeder, E. J., Edwards, A. A., and Dolphin, G. W.; "Chromosome Aberrations Induced in Human Lymphocytes by Radiation from $252 \mathrm{cf}$, "Int. J. Radiat. Biol. 34, 177-186 (1978).

32. Nijhoff, J. H. and deBoer, P.; MRadiation-inducec Meiotic Autosomal Non-disjunction in Male Mice: The Effects of Low Doses of Fission Neutrons and X-rays in Meiosis I and II of a Robertsonian Translocation Heterozygote," Mutation Res. 72, 431-446 (1980).

33. Conger, B. V.; "Maximum RBE of Fission Neutrons for Induction of Somatic Mutations in Maize," Int. J. Radiat. Biol. 27. 271-281 (7975).

34. Smith, H. H. and Rossi, H. H.; "Relation Between Mutation Yield and Cell Lethality Over a Wide Range of X-ray and Fission Neutron Doses in Maize," in: Biological Effects of Meutron Irradiation, IAEA, Vienna (1974), pp. 405-4T6. 
35. Searle, A. G., Evans, E. P. and West, B. J.; "Studies on the Induction of Translocations in Mouse Spermatogonia. II. Effects of Fast Neutron Irradiation, "Mutation Res. 7, 235-240 (1969).

36. Russe11, W. L., "Studies in Mammalian Radiation Genetics," Nucleonics 23, 53-62 (1965).

37. Batchellor, A. L., Phillips, R. J. S., Searle, A. G.; "A Comparison of the Mutagenic Effectiveness of Chronic Neutron and Gamma Irradiation of Mouse Spermatogonia, "Mutation Res. 3, 218-225 (1966.).

38. Searle, A. G.; "Progress in Mammalian Radiation Genetics," Radiation Research (Silini, G., Ed.), North-Holland, Amsterdam (T967) pp. 468-48T.

39. Searle, A. G.; "Genetic Effects of Neutrons in Mamma's and their Implications for Risk Assessment in Man," in: Brclogical Effects of Neutron Irradiation, IAEA, Vienna (1974), pp. 46T-47T.

40. Dobson, R. L. and Kwan, T. C.; "The RBE of Tritium Rejiation Measured in Mouse Oocytes: Increase at Low Exposure Leve1s", Radiat. Res. 66, 615-625 (1976).

41. Dobson, R. L. and Kwan, T. C.; "The Tritium RBE at Low-Leve 1 Exposure-Variation with Dose, Dose Rate, and Exposure Duration," Curr. Top. Radiat. Res. Q. 12, 44-62 (1977).

42. Dobson, R. L., Koehler, C. G., Felton, J. S., Kran, T. C., Wuebbles, B. S., and Jones, D.C.L.; "Vulnerabil ty of Female Germ Cells in Developing Mice and Monkeys to $T$ it ium, Gamma Rays, and Polycyclic Aromatic Hydrocarbons," in: Developmental Toxicology of Energy-Related Pollutants (Mahlum, D. D., Sikov, M. R., Hackett, P. L., and Andrew, F. D., Eds.), U.S. Department of Energy (1978) pp. 1-14.

43. Russe11, W. L.; "The Genetic Effects of Radiation," in: Peaceful Uses of Atomic Energy, United Nations, New York, T(T/2) VOT. T3, pp. 487-500.

44. RusseII, W. L.; "Effect of the Interval Between Irradiation and Conception on Mutation Frequency in Female Mice, ". Proc. Mat' 1 . Acad. Sci. U.S.A. 54, 1552-1557 (1965).

45. Storer, J. B., Serrano, L. J., Darden Jr., E. B., Jernigan, M. C., and Ulirich, R. L.; "Life Shortening in RFM and BALB/C Mice as a Function of Radiation Quality, Dose, and Dose Rate," Radiat. Res. 78, 122-161 (1979). 
46. Dennis, J. A. and Boot, S. J., "Dependence of the Oxygen Enhancement Ratio on Neutron Energy, " Nature (London) 215, 310-311 (1967).

47. Hall, E. J., Rossi, H. H., Kellerer, A. M., Goodman, L. J. and Marino, S.; "Radiobiological Studies with Monoenergetic Neutrons." Radiat. Res. 54, 431-443 (1973).

48. Ellett, W. H. and Blaby, L. A.; "The Microciosimetry of $250 \mathrm{kVp}$ and $65 \mathrm{kVp} \mathrm{X}$-rays, ${ }^{60} \mathrm{Co}$ Gamma-Rays, and Tritium Beta Particles," Radiat. Res. 51, 229-243 (1972).

49. Zamyatnin, Y. S.; "Prompt Neutron Spectrum of the Spontaneous Fission of Californium 252," in: Californium 252 Source Technology, Scientific and Industrial Applications, Proc. Symp., 26-28 April 1976, Paris, France, pp. IV-T to IV-7.

50. Phillips, T. L., Barschall, H. H., Goldberg, E., Fu, K. and Rowe, J.; "Comparison of RBE values of $15 \mathrm{MeV}$ Neutrons for Damage to an Experimental Tumor and Some Normal Tissues," Europ. J. Cancer 10, 287-292 (1974).

51. Oakberg, E. F.; "Gamma-Ray Sensitivity of 0ocytes in Immature Mice," "Proc. Soc. Exp. Biol. Med. 109, 763-767 (1962).

52. Dobson, R.,L. and Cooper, M. F.; "Tritium Toxicity: Effect of Loiv-Level ${ }^{3} \mathrm{HOH}$ Exposure on Developing Female Germ Cells in the Mouse, "Radiat. Res. 58, 91-100 (1974).

53. Ngo, F.Q.H., Antun, H. and Elkind, M. M.: "On the Repair of Sub-Lethal Damage in V79 Chinese Hamster Cells Resulting from Irradiation with Fast Neutrons or Fast Neutrons Combined with X-Rays," Int. J. Radiat. Biol. 32, 507-511 (1977).

54. Railton, ..., Lawson, R. C., and Porter, D.; "Interaction of Gamma Rav and Neutron Effects on the Proliferative Capacity of Chinese tamster Cells," Int. J. Radiat. Biol. 27, 75-82 (1975).

55. Zaider, M. and Rossi, H. H.; "The Synergistic Effects of Different Radiations," Radiat. Res. 83, 732-739 (1980).

56. Watkins, D. K. and Deacon, S.; "Comparative Effects of Electron and Neutron Irradiation on the Release of Enzymes from Isolated Rat-Spleen Lysosomes," Int. J. Radiat. Bioi. 23, 41-50 (1973).

57. Rossi, H. H.; "The Effects of Small Doses of Ionizing Radiation," Phys. Med. Biol. 15, 255-262 (1970).

58. Beaumont, H. M.; "Effect of Hormonal Environment on the Radiosensitivity of Docytes," in: Radiation Biology of the Fetal and Juvenile Mamal, Sikov and Mahtum, Eds., USAEC, ak Ridge (1969), pp. 943-954. 
59. Jones, E. C. and Krohn, P. L.; "The Effect of Hypophysectomy on Age Changes in the Ovaries of Mice," J. Endocrinol. 21, 497-509 (1961).

60. Schneiderman, M. H. and Hofer, K. G.; "The Target for Radiation-Induced Division Delay,." Radiat. Res. 84, 462-476 (1980).

61. Lambert, B.; "Cytological Damage Produced in the Mouse Testes by Tritiated Thymidine, Tritiated Water and X-Rays; "Health Phys, 17, 547-557 (1969).

62. Ord, M. J., and Danielli, J. F.; "The Site of Damage in Amoebae Exposed to X-Rays," Quart. J. Microscop. Sci. 97, 29-37 (1956).

63. Zirkle, R. E., Bloom, W., and Uretz R. B.; "Use of Partial-Cell Irradiation in Studies of Cell Division, " in: Proceedings of the International Conference on the Peaceful Uses of Atomic Energy, United Nations, New York (1956) Vol. 11, pp. 273-382.

4. Rogers, R. W. and von Borstel, R. C.; "Alpha-Particle Bombardment of the Habrobracon Egg. I. Sensitivity of the Nucleus," Radiat. Res. 7, 484-490 (1957).

65. Brandt, W. and Ritchie, R. H.; "Primary Process in Physical Stage," in: Physical Mechanisms in Radiation Biology, Proceedings of a Conference in ArTie, Virginia $11-14$ october 1972, USAEC Technical Information Center, pp. 29-46.

66. Baker, T. G. and McLaren, A.; "The Effect of Tritiated Thymidine on the Developing Oorytes of Mice," J. Reprod. Fert. $34,121-130$ (1973).

67. International Commission on Radiation Units and Measurements, "Linear Energy Transfer," ICRU Rept. 16, Washington D.C. (1970).

68. Okada, S.; "Radiation Biochemistry: Cells," (K. J. Altman, G. B. Gerber and S. Okada, Eds.), Academic Press, New York (1970), pp. 3-39.

69. Kuppermann, A.: "Diffusion Kinetics in Radiation Chemistry: An Assessment, " in: Physical Mechanisms in Radiation Biology, Proceedings of a Conference in Arlie, Virginia Ti-14 October 1972, USAEC Technical Information Center (1974) pp. i55-176.

70. Rossi, H.H.: "Microscopic Energy Distribution in Irradiated Matter," in: Radiation Dosinetry, F. H. Attix and W. C. Roesch, Eds., Academic Press, New York, Vol. 1 (1968), pp. 43-92.

71. International Comission on Radiation Units and Measurements, "Average Energy Required to Produce an Ion Pair, "ICRU Report 31, Washington, D.C. (1979). 
72. Auxier, J. A., Snyder, W. S., and Jones, T. D.; Meutron Interactions and Penetration in Tissue," in: Radiation Dosimetry (F. H. Attix and W. C. Roesch, Eds.), AcademfC Press, New York (1968), pp. 275-316.

73. Dobson, R. L.; Personal Communication.

74. Parsons, D. F.; "An Electron Microscope Study of Radiation Damage in the Mouse Oocyte," J. Cell Biol. 14, pp. 37-48, (1962).

75. Rossi, H. H. and Mays, C. W.; "Leukemia Risk from Neutrons," Health Phys. 34, 353-360 (1978).

76. Loewe, W. E. and Mendelsohn, E.; "Revised Dose Estimates at Hiroshima and Nagasaki," Health Phys, 41, 663-666 (1981).

77. Kerr, G. D., "Review of Dosimetry for the Atomic Bomb Survivors," in: Proceedings of the Fourth Symposium on Neutron Dosimetry, Comission of the European Communities, Luxembourg तT81) V0i. 1, pp. 501-513.

78. Auxier, J. A., Cheka, J. S., Haywood, F. F., Jones, T. D. and Thorngate, J. H.; "Free-Field Radiation-Dose Distributions from the Hiroshima and Nagasaki Bombings," Health Phys. 12, 425-429 (1966).

79. Milton R. C. and Shohoji, T.; "Tentative 1965 Radiation Dose Est imation for Atomic Bomb Survivors," Atomic Bomb Casualty Commission Report TR-1-68 (7968).

80. Beebe, G. W., Kato H., and Land, C. E.; "Studies of the Mortality of A-Bomb Survivors: 6 . Mortality and Radiation Dose, 1950-1974," Radiat. Res. 75, 138-201 (1978).

81. Medina, D.; "Preneoplastic Lesions in Mouse Mammary Tumorigen... " in: Methods in Cancer Research, H. Bush, (Ed.), Acac. : Press, New York (1973) pp. T-53 (1973).

82. Ohkita, T. and Kamada, N.; "Leukemia Among Atomic Bomb Survivors, " in: Proceedings of the Sixth International Congress of Radiation Research, Japanese Association for Radiation Research, Tokyo (1979) pp. 59-68.

83. Kaplan, H. S.; "Interaction Between Radiation and Viruses in the Induction of Murine Thymic Lymphomas and Lymphatic Leukemias," in: Radiation-Induced Leukemogenes is and Related Viruses, J. F. Duptan (Ed.), North-Hotland Publishing Company, Amsterdam (1977) pp. 1-18.

84. Schul1, W. J.,Otake, M., and Neel, J. V.; "Genetic Effects of the Atomic Bombs: A Reappraisal," Science 213, 1210-1227 (1981). 
85. Russe11, W. L.; Mutation Frequencies in Female Mice and the Estimation of Genetic Hazards of Radiation in Women, "Proc. Nat 1. Acad. Sci. USA. 74, 3523-3527 (1977).

86. Report of the RBE Committee to the International Commissions on Radiological Protection and on Radiological Units and Measurements, Health Phys. 9, 357-386 (1963).

87. International Commission on Radiation Units and Measurements, Radiation Quantities and Units, ICRU Report 33, Washington, D.C. (1980).

88. National Council on Radiation Protection and Measurements, NCRP Statement on Dose Limit for Neutrons, Washington, D.C. (1980).

89. Bond, V. P.; "Quantitative Risk in Radiation Protection Standards," Radiat. Environ. Biophys. 17, 1-28 (1979).

90. Rossi, H. H.; "A Proposal for Revision of the Quality Factor;" Radiat. Environ. Biophys. 14, 275-283 (197?).

91. Reevaluations of Dosimetric Factors, Hiroshima and Nagasaki, J. W. Thiessen and V. P. Bond (Eds.), DOE Symposium Series, CONF-810928, National Technical Information Service, Springfield, Virginia (in press).

92. ICRP Report 27, Problems Involved in Developing an Index of Harm, The International Commission on Radiological Protection, Pergamon Press, New York (1977).

93. Loewe, H. E.; Personal Communication. These values are estimates (not specifically computed) by Dr. Loewe and represent his expert judgement in this field.

94. Searle, A. G.; "Mutation Induction in Mice," Adv. in Radiat. Biol. 4, 151 - 160 (1974).

95. Kerr, G. D.; "Organ Dose Estimates for the Japanese Atomic Bomb Survivors," Health Phys. 37, 487 - 508 (1979).

96. Mole, R. H. and Davids, J.A.G.; "Induction of Myeloid Leukemia and Other Tumors in Mice After Irradiation with Fission Neutrons," in: Proceedings of European Seminar on Neutron Carcinogenesis, Rijswijk, The Netherlands (1982), in-press.

97. Wuebbles, B; Radiation Damage in the Mouse Female Germ Ce11: A Two-Part Study, Lawrence Livermore National Laboratory Report, . UCRL-52530 (Juiy 1978).

98: A Textbook of Histology, Bloom, W. and Fawcett, D. W. (Eds.), W. B. Saunders Publishing Company, Philadelphia (1975) pp. 54-57. 
APPENDIX 1

IMPLICATIONS OF NEW HIROSHIMA AND NAGASAKI DOSE ESTIMATES: CANCER RISKS AND NEUTRON RBE

T. Straume and R. Lowry Dobson

Hazards Control Department and Biomedical Sciences Division Lawrence Livermore National Laboratory, P.0. Box 808, Livermore, CA 94550

Radiation doses delivered by the atomic bombs in Hiroshima and Nagasaki have recently been recalculated by W.E. Loewe and E. Mendeisohn at this Laboratory (LLNL) (LOBI). The new est imates are substantially different from the generally accepted T65 doses (Mi68, Au77), and indicate that the gamma-ray component and totai dose were higher in Hiroshima than previously believed and lower in Nagasaki, while the neutron components were lower in both cities. There remain uncertainties in the doses, and work on them continues. But the uncertainties are thought to be less than a factor of two for neutrons and even smaller for gamma rays (L081). Shielding factors for japanese buildings and the human body have not yet been recalculated; they will be different now, in part because output spectra used for the new doses are not the same as those assumed for the T65 doses. These shielding factors will be needed for determining actual absorbed doses. In the interim, however, the factors previously derived for T65 doses (Mi68, Ke79) can be used as a first approximation, as we have done in the analyses presented here.

We have applied the new LLNL dose estimates to available biomedical data from A-bomb survivors (Be78, To79, Aw78) and reanalyzed dose-response relations for four major endpoints: radiation-induced leukemia, breast cancer, total malignancies, and chromosome aberrations. From the results, inferences are drawn regarding risk coefficients for radiation-induced malignancies and, where possible, regarding neutron RBE.

\section{LEUKEMIA}

When the new LLNL doses are combined with Hiroshima and Nagasaki data for leukemia (Be78), dose-response curves are sigmoidal for both cities and not significantly different from each other. This is seen in Fig. 1, where the data for the two cities, not differing significantly, are well fit by a single curve. This contrasts with what was previously thought to be the case. When T65 doses are used, the two curves are separate and differ in shape; and this has been interpreted as reflecting high neutron RBE at low doses (Ro78). With 
the LLNL doses, however, the lack of difference between the Hiroshima and Nagasaki curves suggests a low-dose neutron RBE, for leukemia induction, close to unity, although an upper limit of about 25 is compatable with the data.

Risk coefficients for radiation-induced leukemia determined with the LLNL doses are shown in Table 1. At low doses, the coefficient is consistent with the risk factor given by ICRP for lOW-LET radiation (ICRP77). The ICRP lifetime risk factor of $2 \times 10^{-3}$ $\mathrm{Sv}^{-1}$, with 25 years assumed as the period of excess leukemia risk, is equivalent to the risk coefficient of $0.8 \times 10^{-6}$ cases per person-year-rad, which is given in Table 1 . At high doses the coefficient reaches a value more than four-fold greater than the ICRP risk factor. In Table 2 are given the doubling dose and values for relative risk.

\section{BREAST CANCER}

When the LLNL doses are applied to Hiroshima and Nagasaki breast-cancer data (To79), response curves for the two cities, while showing suggestive differences at low doses, are well represented by a single curve ( $x^{2}$ per d.f. $=0.4$, indicating excellent $\left.f i t\right)$, as seen in Fig. 2. Again, neutron RBEs different from unity cannot be demonstrated and, due to greater statistical uncertainties in the breast-cancer data, an upper limit for the RBE is even less clear than it is for leukemia. The similarity in shape of the leukemia and breast-cancer curves, except for a steeper initial slope for breast cancer, resuit in similar risk coefficients, shown in Table 1. Note, however, that the doubling dose for leukemia (given in Table 2) is considerably smaller than that for breast cancer and the relative risk is larger at all doses.

\section{TOTAL MALIGNANCIES}

In contrast to the situation for leukemia and breast cancer, dose-response curves for total (combined) malignancies clearly demonstrate significant inter-city differences. These are apparent in Fig. 3. (Differences for total malignancies are seen also when T65 doses are used.) If one assumes that the inter-city differences in dose-response seen with the LLNL doses are due to the larger neutron component in Hiroshima than in Nagasaki (L081), neutron RBEs can be determined by the method of Ross $i$ and Mays (Ro78). In this way, and using statistical refinements to be described elsewhere (manuscript in preparation), we obtain a dose-response curve for Hiroshima neutrons, which is shown in Fig. 4 together with the curve for Nagasaki garma rays. The RBE is the ratio of gamma-ray and neutron doses for equal effect. Its value is seen to increase with decreasing dose. (It should be noted that the increase in RBE is not due to enhanced neutron effectiveness at low doses, but to diminished gamma-ray effectiveness.) At very low doses, the RBE reaches high values. Although large uncertainties are involved, an RBE of up to 
100 or more cannot be excluded, as seen in Fig. 5. (Neutron RBEs obtained in this manner for total malignencies do not differ greatly for LLNL and T65 doses.)

If leukemia, which shows no significant inter-city differences (Fig. 1), is removed from these total-malignancy data, an even greater contrast is found between Hiroshima and Nagasaki. Interestingly, the risk coefficient for the remaining cancers is extremely low for gamma rays at doses below $100 \mathrm{rad}$. The risk coefficients listed in Table 1 already reflect this phenomenon, with coefficients for total malignancies being lower than those for leukemia alone. This apparent inconsistency will be further considered elsewhere (manuscript in preparation).

\section{CHROMOSOME ABERRATIONS}

Data for chromosome aberrations in A-bomb survivors (Aw78), when similarly analyzed with LLNL doses for dose-response relations, again clearly demonstrate significant inter-city differences. This is seen in Fig. 6. (Similar differences appear a 7 so with T65 doses.) Using the methods referred to above (RO78), neutron RBEs for chromosomeaberration induction have been estimated with the ne'N LLNL doses. The RBE increases with. decreasing dose and, at very low doses, reaches values similar to those for total malignancies, as seen in Fig. 5. (RBEs determined with T65 doses are again not inconsistent with this.)

\section{DISCUSSION AND CONCLUSIONS}

What has seemed up to now a curious incompatability between the Japanese and other human radiobiological data is resolved by the new doses. Brown (Br77) had noted that A-bomb data (with T65 doses) gave risk coefficients for both leukemia and breast cancer that were unexpectedly low for acute exposure when compared with risk coefficients for chronic and fractionated medical exposures. However, the coefficients derived here with LLNL doses (Table 1) largely remove this inconsistency. The re-evaluated A-bomb-survivor data do not support the argument that low doses or low dose rates carry higher risk per rad than high doses or high dose rates. Rather, the contrary is observed with the new LLNL dose estimates; and this is consistent with experimental radiobiological results.

Our conclusions from these preliminary analyses can be summarized as follows:

- The new LLNL doses fit well with epidemiological observations in Japan, and dose-response relations derived from them give improved consistency between A-bomb and other radiobiological data. 
- Risk coefficients for radiation-induced leukemia and breast cancer obtained from the new dose-response curves are consistent--at iow doses--with those of ICRP. The TOW-LET coefficients for total malignancies appear lower than the ICRP value.

- Evidence for high neutron RBE is lacking for leukemia and breast cancer. However, data for total malignancies and for chromosome aberrations suggest that neutron RBES increase with decreasing dose, because of the diminishing effectiveness of gamia rays, and may, reach quite high values (100 cannot be excluded).

- The contrasting neutron RBEs observed for leukemia and breast cancer, on the one hand, and for total malignancies, on the other, need to be clarified.

\section{ACKNOWLEDGMENT}

We thank Drs. M.L. Mendelso, W.E. Loewe, H. Kruger, and Mr. E. Mendelsohn for discussions during the preparation of this report, and Dr. D. Moore for statistical advice. This work was performed under the auspices of the U.S. Department of Energy by the Lawrence Livermore National Laboratory uncer contract number W-7405-Eng-48. 


\section{REFERENCES}

Au77 Auxier J.A., 1977, "Ichiban: Radiation Dosimetry for the Survivors of the Bombings of "Airoshima and Nagasaki," (ERDA Technical Information Center, Oak Ridge).

Aw78 Awa A.A., Sofuni T., Honda T., I toh M., Neriishi S., and Otake M., 1978, "Relationship Between the Radiation Dose and Chromosone Aberrations in Atomic Bomb Survivors of Hiroshima and Nagasaki," J. Radiat. Res. 19, 126-140.

Be78 Beebe G.W., Kato H.s and Land C.E., 1978, "Studies of the Mortality of A-Bomb Survivors: 6. Mortality and Radiation Dose, 1950-1974," Radiat. Res. 75, 138-201.

Br77 Brown J.M., 1977, "The Shape of the Dose-Response Curve for Radiation Carcinogenes is: Extrapolation to Low Doses," Radiation Res. 71, 34-50.

ICRP77

International Commission on Radiological Protection, 1977, "Radiation Protection," ICRP Publication 26 (0xford, Pergamon Press).

Ke79 Kerr G.D., 1979, "Organ Dose Estimates for the Japanese Atomic-Bomb Survivors," Health Phys. 37, 487-508.

L081 Loewe W.E. and Mendelsohn E., 1981, "Revised Dose Estimates at Hirosinima and Nagasaki," Health Phys. (in press).

Mi68 Milton R.C. and Shohoji T., 1968, "Tentative 1965 Radiation Dose Estimation for Atomic Bomb Survivors," Atomic Bomb Casualty Commission Report TR-1-68.

Ro78 Rossi H.H. and Mays C.W., 1978, "Leukemia Risk from Neutrons," Health Phys. 34, 353-360.

To79 Tokunaga M., Norman J.E., Jr., Asano M., Tokuoka S., Ezaki H., Nishimori I., and Tsuji Y., 1979, "Malignant Breast Tumors Among Atomic Bomb Survivors, Hiroshima and Nagasaki, 1950-1974, " J. Nat 1. Cancer Inst. 62 (6), 1347-1359. 
TASLE 1. Risk coefficients for leukemia and total malignancies (mortality) and for breast cancer (incidence) at selected absorbed doses.

\begin{tabular}{|c|c|c|c|}
\hline \multirow{2}{*}{$\begin{array}{l}\text { Induced } \\
\text { effect }\end{array}$} & \multicolumn{3}{|c|}{ Risk coefficient* } \\
\hline & $10 \mathrm{rad}$ & $100 \mathrm{rad}$ & $200 \mathrm{rad}$ \\
\hline Leukemia & $0.8(-1.2$ to 2.8$)$ & $2.7(1.4$ to 3.9$)$ & $4.8(2.8$ to 6.8$)$ \\
\hline $\begin{array}{l}\text { Breast } \\
\text { cancer }\end{array}$ & $1.4(-3.6$ to 6.4$)$ & $2.4(1.1$ to 3.7$)$ & $3.3(2.3$ to 4.3$)$ \\
\hline $\begin{array}{l}\text { Total } \\
\text { malignancies }\end{array}$ & $-0.7(-1.2$ to 1.6$)$ & $1.1(-1.5$ to 3.7$)$ & $5.8(3.3$ to 8.3$)$ \\
\hline
\end{tabular}

TABLE 2. Doubling doses and relative risks for leukemia and total malignancies (mortality) and for breast cancer (incidence) at selected absorbed doses.

\begin{tabular}{lcccc}
\hline & \multirow{2}{*}{$\begin{array}{l}\text { Doubling } \\
\text { dose, rad } \\
\text { effect }\end{array}$} & $10 \mathrm{rad}$ & $100 \mathrm{rad}$ & $200 \mathrm{rad}$ \\
\cline { 5 - 5 } & 27 & 1.3 & 8.6 & 28 \\
\hline $\begin{array}{l}\text { Leukemia } \\
\begin{array}{l}\text { Breast } \\
\text { cancer }\end{array}\end{array}$ & 80 & 1.1 & 2.4 & 4.4 \\
$\begin{array}{l}\text { Total } \\
\text { malignancies }\end{array}$ & 246 & $\sim 1.0$ & 1.2 & 1.7 \\
\hline
\end{tabular}

*The ratio of total cases to naturally occurring cases ( $Y$-intercepts . of curves in Figs. 1, 2, and 3 taken as natural occurrence rates); Nagasaki curve (TOW-LET rádiation) used for total malignancies. 
Fis. 1. I.eukente eortality in the two cfeles show as anction of LWWL eat lestes of dowe to boue anrrou. llere and in Fige $2,3,4$, and 6 , solld curves are ilnear-quadratic, leset-squares bet fits to the date poines, wich ench point wetgheed by 1/30; vertleal bere ere \pm 130. Dahed curves extend beyond the data used for fitting and in wist cases Indicate dowmerd curvature frob cell-k11110 affect at highar doese (however, even if the high-loen polate are Included in the fleting tbey do not appreetably affect curve ahapes below 100 rad, the regiene of grentest intereet).

F18. 2. Breat cancer Incidence in the two citles thow at function of LWML estaces of done to breast.
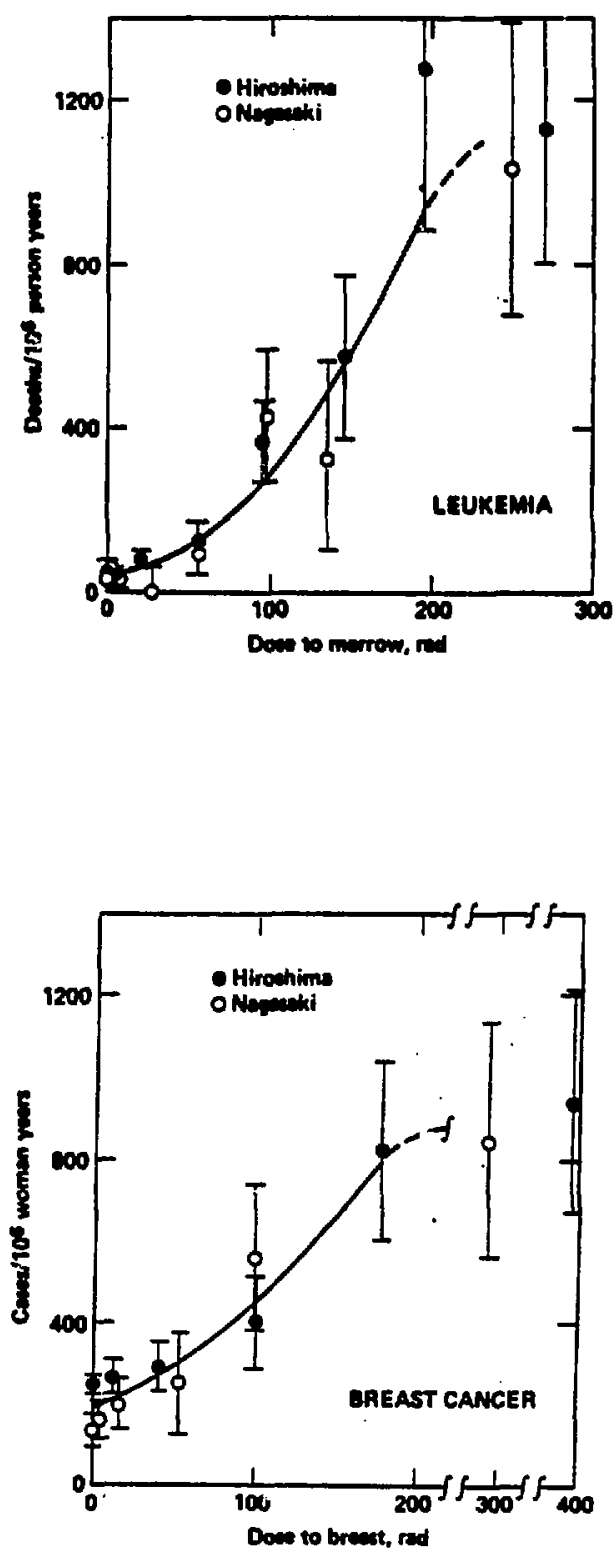


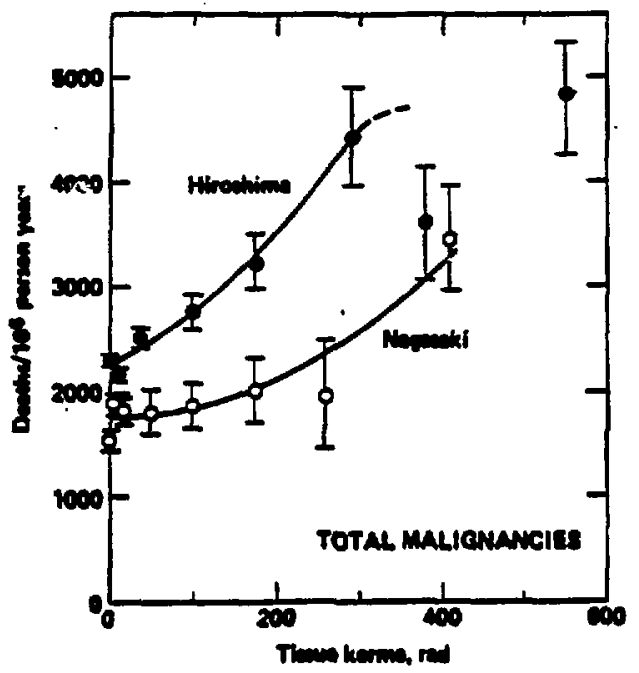

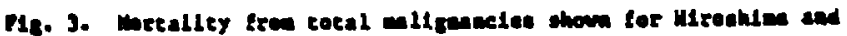

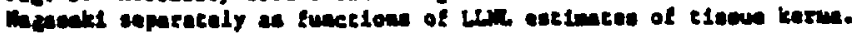

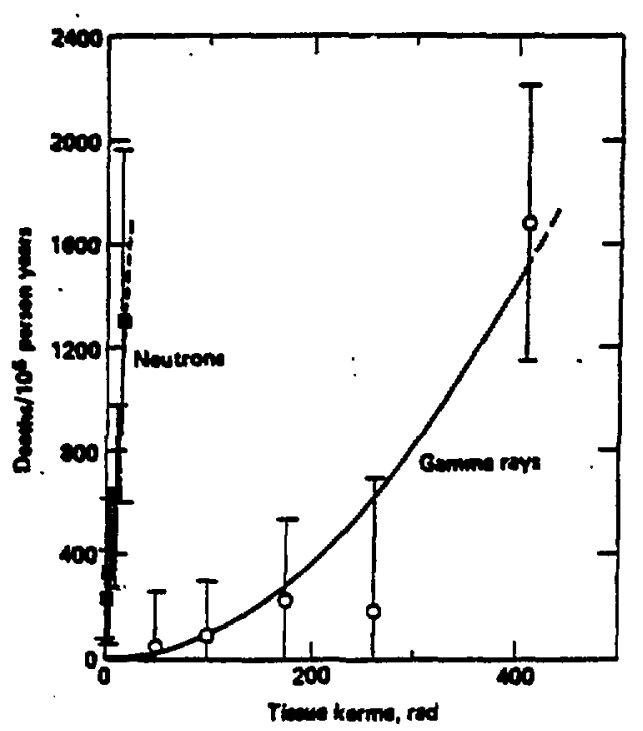

18. 4. Dose-response curves for neutrons and pane ray coupared; andpotat used, net eot talfe; fron cotal enliganedes. 


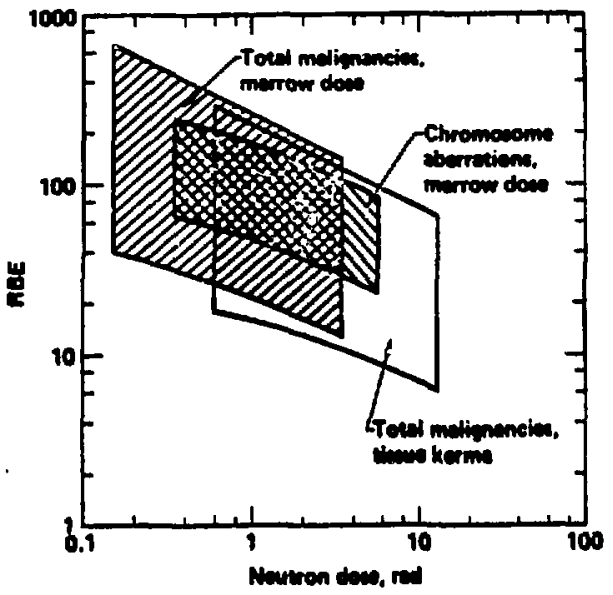

F1y. 5. Rance of probble newtron atse tor cocal callgancies (lor

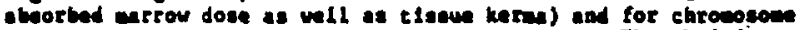
aberuten, shown as functions of nutren doee. The shaded arans represent ranges of uncertaln: derived by prepagating errors in ere

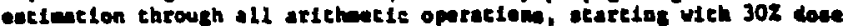

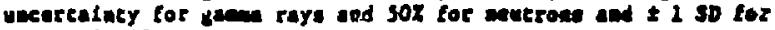
oluerved offects.

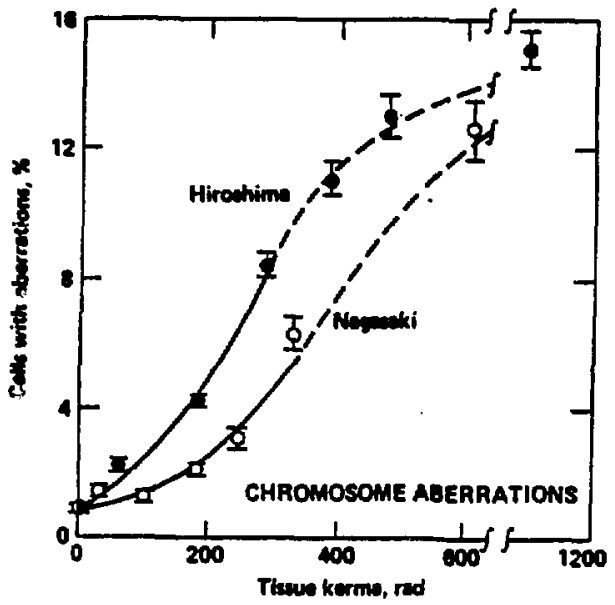

718. 6. Chrososowe aberraclone (exchanges) Ia blood lyephocytea shown for each elty as a function of Lhe onctented clesue keren. 
APPENDIX 2

\title{
SENSITIVITY OF HIROSHIMA AND NAGASAKI EPIDEMIOLOGICAL INFERENCES TO DOSIMETRIC PARAMETERS
}

\author{
M.L. Mendelsohn*, T. Straumet, and R.L. Dobson* \\ *Biomedical Sciences Division and \\ thazards Control Department \\ Lawrence Livermore Nationa? Laboratory \\ University of California \\ Livermore, California
}

\begin{abstract}
An analysis is made here of the influence that various possible values of physical dosimetric parameters can have on radiobiological interpretations of Hiroshima and Nagasaki epidemiologic data. The physical paraneters studied, which include yields of the weapon devices, neutron and garma-ray outputs, and slopes of the dose-versus-distance functions, are al lowed to vary over reasonable ranges and the resulting effects on a representative biological endpoint are evaluated. The endpoint used is radiation-induced chronosome aberrations in blood cells of A-bomb survivors. While this is - aiistically perhaps the strongest set of biomedical observations available for the purpose, it is used here simply as an indicator endpoint for exploring the jiological consequences of varying the physical parameters.

Neutron RBEs calculated from the resulting dose-response relationships are found to be most sensitive to variations in device yield, with a sensitivity ratio (SR) of 1.2 (12\% change resulting from 10\% parameter change). RBEs are also very sensitive to Hiroshima neutron output alone $(S R=0.7)$. They are least sensitive to Hiroshima gamma-ray output $(S R=0.4)$. Gamma-ray risk coefficients, which depend only on Nagasaki data, are in"ersely proportional to the Nagasaki gamma-ray output (and device yield).

Based on the assumption that neutrons could not have been protective, results from this analys is suggest limits on certain physical paraneters--device yields and gamma-ray outputs for both cities. No such limits, however, are found for reasonable values of Hiroshima neutron output, and this parameter has the potential for strongly influencing inferences regaroing neutron RBE.
\end{abstract}




\section{INTRODUCTION}

Radiation biology and radiation epidemiology can play significant roles in the evaluation of past and present dosimetry at Hiroshima and Nagasaki, as well as in the planning of projected dosimetric work. These disciplines can contribute to setting goals for statistical confidence limits of the relevant physical data, to defining zones of reasonable dosimetric outcome, and perhaps to m:aking choices among physical alternatives. They may also clarify some of the ongoing and confused public debate about the impact of recent changes in the dosimetry on issues of radition standards and regulatory policy.

The new radiation dose estimates for Hiroshima and Nagasaki (Loewe and Mendelsohn, 1981a; Kerr, 1981) differ significantly from the previously accepted T650 estimates (Auxier, 1966, 1977), and further dosimetric work is actively underway by a number of investigators aimed at establishing with acceptable precision what the doses were. It is especially profitable at this stage, therefore, while further dosimetry is being planned and pursuer, to systematicaliy examine both the magnitude and range of effects that various possible values of physical dosimetric parameters can have on radiobiological interpretations of the data from A-bomb survivors. Such an examination is made here in an attempt to identify the parameters in need of greatest precision and to provide a clearer perspective on the relationship between the physics and the biology.

Chromosome aberrations enumerated in blood lymphocytes of persons exposed at Hiroshima and Nagasaki were selected as the radiation-effect endpoint for this analysis. The data for this particular endpoint are especially extensive (Awa, 1978) and constitute probably the most statistically robust set of observations available. Furthermore, chromosome aberrations are biologically important; they indicate damage to genetic structures and riay be involved in carcinogenesis. It should be emphasized, however, that for present purposes this data set was selected simply as a representative model biolcgical endpoint. We are concerned here nut with any specific dose-response relationship, but with elucidating how such relationships depend upon, and vary with, dosimetric parameter values.

The physical parameters studied include yields of the two weapon devices, neutron output of the Hiroshima device, slope of the Hiroshima neutron dose-versus-distance function, gamma-ray outputs, and slope of the gamma-ray dose-versus-distance function. Neutron RBEs and ganma-ray risk coefficients are calculated from the birlogical data for the various parametric assumptions and compared with corresponding data in the radiobiological literature. 
METHODS

\section{Biological Endpoint and Curve Fitting}

Data for chromosomal exchanges are from fwa's (1978) report on radiation-induced chromosome aberrations in A-bomb survivors. In the present study attention is confined to exchanges, as they are least susceptible to uncertainties; data for other aberrations recorded by Awa (1978) are not included in this analysis.

For fitting basic dose-response curves to the biological data, linear-quadratic, least-squares best fits are employed, with data points weighted by $1 /(S D)^{2}$, where SO $=100 \times$ (number of cells with exchanges) $/ / 2$ /number of ceils examined. Additional standard deviations reflecting interpersonal differences were not given by Awa (1978). For both Hiroshima and Nagasaki, only the lower portions of the curves are used for analysis---the lower 4 data points for Hiroshima and the lower 6 points for Nagasaki, as shown in Fig. 1 by solid curves. Above these fitted regions inflections occur as the curves change from positive to negative curvaturn (presumabily reflecting cell-killing effects). The higher data points, eye-fitted with dashed curves in Fig. 1 , are not used in the analysis. Confidence limits for the biological data, except as employed in curve fitting, are not considered here as they are not relevant to the analysis of effects of dosimetric parameter variation.

\section{Reference Dosimetric Parameters}

The values for physical dosimetric parameters given by Loewe and Mendelsohn (1981a) are taken here, quite arbitrarily, as reference values for the independent variables used in the analysis. This particular reference selection was for convenience only; other estimates, such as those of Auxier (1966) could serve equally well.

Transmission Factors for Japanese Houses and the Human Body

Although questions concerning transmission-factor values are not directly relevant to our principal concern here, they are important to relating neutron RBEs from the Japanese data to those found experimentally in the laboratory. New transmission factors determined for the reference energy snectra (Loewe and Mendelsohn, 1981b) have not yet been generally agreed upon and are current ly being investigated. However, it is desirable for the present and?ysis to derive approximate values in order to estimate the impact that the new spectra could have on the total transmission factors (i.e., Japanese houses and human body) used here. New values for Japanese houses have been estimated by Marcum (1981), and are shown in Table 1. For the human body, we derived transmission factors by applying published values (Jones, 1977; Kerr, 1979) to estimated averages of the reference gamina-ray energy spectra for prompt, fission-product, and n-capture ganma rays. For neutrons, we assumed 
that T650 body transmission factors remained unchanged, as Marcum (1981) found for Japanese houses (mostly wood firame) that no significant changes are indicated. From this preliminary analysis, it appears that total transmission factors (for houses plus the human body) for converting free-in-air tissue kerma to bone-marrow dose will not be greatly different from those of $T 650$, as illustrated in Täble 1 .

Hence, for the present investigation, dose to bone marrow (chosen as reasonably representative for sites of lymphocytic stem cells) was calculated from the reference free-in-air tissue kerma by using attenuation factors derived for T65D specira, as given by Milton and Shohoji (1968) for Japanese houses and by Kerr (1979) for average Japanese anatomical features. However, mean values for each city, as given by Milton and Shohoji (1968), were not used; rather, more range-specific values were obtained from their data by taking into account regional differences in shielding described by them for various distances.

Neutron RBE Calculation

RBEs for Hiroshima neutrons were determined by the method of Rossi and Mays (1978). The principle of this method is presented in Table 2. For the gamma-ray component of each Hiroshima data point, an identical garma-ray dose, together with its associated effect and (sma11) neutron dose component, is obtained by interpolation from the fitted Nagasaki dose-response curve (which represents nearly pure gamma rays). The Nagasaki values are then subtracted from the Hiroshima values, leaving only residual Hiroshima neutron ciose and its corresponding residual effect. From these residual values a neutron dose-response curve is constructed. Neutron RBEs are then obtained from fitted dose-response curves (assumed linear for Hiroshima neutrons and linear-quadratic for the Nagasaki radiation) by taking the ratios of equal-effect doses.

\section{RADIOBIOLOGICAL CONSEQUENCES OF VARYING DOSIMETRIC PARAMETERS}

\section{Effects on Neutron RBE}

Yield of Hiroshima Device. To examine the influence that differing assumed values of Hiroshima yield can have on neutron RBE, the yield was allowed to range from 10 to $25 \mathrm{kilotons,} \mathrm{encompassing} \mathrm{both} 15 \mathrm{kT}$, the value recently proposed by Loewe and Mendelsohn (198ia) and 12.5 $k T$, the value used in the T65D estimates (Auxier, 1966, 1977) and also proposed by Kerr (1981). In Fig. 1, dose-response curves for chromosomal exchange aberrations versus total radiation dose to bone marrow are shown for 10,15 , and $20 \mathrm{kT}$. At $10 \mathrm{kT}$ the Hiroshima and Nagasaki curves differ greatly from each other. Their divergence is less at a device yield of $15 \mathrm{kT}$. The Hiroshima curve continues to move to the right as yield increases further, and the two curves become almost indistinguishable at $20 \mathrm{kT}$. 
From such curves, dose-response relationships for neutrons were derived as described in METHODS. A set of such relationships is shown in Fig. 2 for Hiroshima yields of 10 to $25 \mathrm{kT}$. They are reasonably linear, particularly at lower kT (linearity is expected for neutrons (Lloyd et al., 1976)). At $25 \mathrm{kT}$, however, there are difficulties: not on Ty is there nonlinearity, but the function is negative, which is biologically absurd. From the radiubiological relationships, then, it appears that a value of about $20 \mathrm{kT}$ is an upper limit of plausible Hiroshima yield.

The neutron dose-response relationships, linearly fitted, were used to derive corresponding neutron RBE values, and the systematic. dependence of RBE on Hiroshima yield is shown in Fig. 3. The function is seen to be quite steep for small neutron doses, 3BE $r$ ising as relative Hiroshima yield decreases. The question hen is: Are any of these RBE values radiobiologically plausible? This is addressed in Fig. 4, where neutron RBEs for Hiroshima yields of 10 , 15, and $20 \mathrm{kT}$ are displayed as functions of neutron dose in a jiagram that also shows, for comparison, laboratory results from four radiobiological endpoints for which extensive experimental data are available (Rossi, 1980), including a malignancy (in the $r a t$ ), mutations (in a plant), and chromosome aberrations (in human 1 ymphocytes irradiated in vitro). As seen in Fig. 4, neutron RBEs calculated from the Hiroshima-Nagasaki data are higher than expected from the laboratory results. The A-bomb RBEs approach the experimental RBEs as yield increases, but even at the upper limit of biologically plausible Hiroshima yield, they remain above the experimentally expected values.

Yield of Nagasaki Device. The Nagasaki yield was allowed to range from 17.5 to $24 \mathrm{kT}$, encompassing the generally accepted value of $22 \mathrm{kr}$ (about which there exists no serious debate), while all other parameters were held constant at the reference values. Neutron RBEs calculated for various Nagasaki yields are shown in Fig. 5, again plotted with laboratory data for comparison. The influence of Nagasaki yield on RBE is opposite to that of Hiroshima yield (compare Figs. 4 and 5), but of similar magnitude; the values again lie well above literature values for all acceptable and reasonable yields. The data for neutron dose-response curves are not shown, but below 17.5 kT the curves become negative indicating a lower limit for the Nagasaki yield.

Neutron Output of Hiroshima Device. The Hiroshima neutron output was allowed to range from 0.5 to 5 times the reference value; all other parameters, including Hiroshima yield, being held constant. Representative neutron dose-response curves derived from the resulting relationships are shown in Fig. 6, and the systematic dependence of RBE on Hiroshima neutron output, for representative doses of 1 and $10 \mathrm{rad}$, is shown in Fig. 7. For small doses the function is quite steep, especially at lower neutron outputs. The overall effect of neutron output on RBE is seen in Fig. 8, where RBE 
in the 1 to 10-rad range of neutron dose is shown for a series of assumed neutron outputs. Interestingiy, for outputs $\hat{3}$ to 5 -fold higher than the reference value (intermediate between reference and T65), calculated RBEs lie very close to or within the rance of laboratory experimental data. No biological constraints were found for any reasonable Hiroshima neutron output values.

Slope of Hiroshima Neutron Dose-Versus-Distance Function. To explore the influence of this parameter, two approaches were used. In the first, referred to as "tilt-1" and illustrated in Fig. 9, the slope of the dose-versus-distance function was varied, all resulting curves having common origin at zero slant range (i.e., at the exploding device). In the second, referred to as "tilt-2" and illustrated in Fig. 12, the function was allowed to pivot about a common point at a slant range of interest, thus allowing exploration of greater differences in slope.

For tilt-1, the reference neutron dose-versus-distance function (Loewe and Mendelsohn, 1981b), which is shown in Fig. 9 as a solid curve, was first approximated by a straight line (dashed line 1 in Fig. 9). Then lines of comon origin but differing slope were considered (two are shown in Fig. 9, dashed and labeled 0.5 and 2 ). The numerical values assigned to the considered lines, e.g., $0.5,1$, and 2 shown in Fig. 9, refer not to the slopes themselves, but to relative neutron dose (compared to the reference value on the solid curve) at a particular (but arbitrary) slant range of interest, viz., $1.28 \mathrm{~km}$, corresponding to the third data point of the fitted Hiroshima dose-response curves in Fig. 1 .

Neutron dose-response curves derived for relative tilt-1 of 0.5 to 2 are seen in Fig. 10. By comparing Figs. 10 and 6 it will be noted that the tilt-i approach gives results very similar to those for variations in neutron output. This is a reflection of the slope similarity of the lines in Fig. 9. Over the limited slant range of interest (indicated on the abscissa in Fig. 9), lines $0.5,1$, and 2 do not appear for practical purposes very different from. parallel. The resulting similarity in results is graphically seen in Fig. 11, where neutron dose-response curves for various neutron outputs and for various $t i l t-l$ values are plotted together. The effects of variations in tilt-i on neutron RBE are quite similar to those shown in Fig. 7 and 8 for variations in Hiroshima neutron output.

For tilt-2, the reference function, shown in Fig. 12 as a solid curve, was again first approximated by a straight line (dashed 1 ine 1 in Fig. 12). Then lines of significantiy differing slope, intersecting at a point corresponding to the middie of the biological data range (indicated on the abscissa in $F i g .12$ ) were considered. Numerical values assigned to the lines, e.g., $0.5,1$, and 2 in Fig. 12, refer not to slopes but to relative neutron doses at $1.54 \mathrm{~km}$ slant range, the distance corresponding to the second Hiroshima data point used for fitting the dose-response curves of Fig. $T$. 
The influence of $t i 1 t-2$ on neutron RBE is summarized in Table 3. RBE is not a strong function of tilt-2 variation; it changes only from about 150 to 100 for an unrealistically exaggerated range of relative tilt-2 values. (That this range is unrealistic is shown by neutron outputs corresponding to extrapolations (to zero s lant range) of 7 ines 0.5 and 2 of $F i g .12$; they correspond respectively to 200 and 0.004 times the reference value, well exceeding reasonable limits.)

Hiroshima Gamma-Ray Output. In Fig. 13 are presented a group of neutron dose-response curves derived from relationships for various assumed Hiroshima gamma-ray cutputs. RBE dependence on this parameter is shown in Fig. 14 for representalive neutron doses of 1 and $10 \mathrm{rad}$, and in Fig. 15 RBEs for various assumed Hiroshima gamma-ray outputs are compared with laboratory radioblological results. From Figs. 14 and 15 RBE is seen to be only weakly influenced by variations of Hiroshima gamma-ray output. For relative gamma-ray outputs exceeding 1.25 the neutron dose-response curve becomes biologically meaningless.

Nagasaki Gamma-Ray Output. This parameter affects RBE the same as yield of Nagasaki device.

Slope of Hiroshima Gamma-Ray Dose-Versus-Distance Function. In a manner similar to that used for analyzing effects of Hiroshima neutron tilt-1, the slope of the Hiroshima gamma-ray dose-versusdistance function was al lowed to vary so that gamma-ray doses at a particular slant range of interest $(1.28 \mathrm{~km}$, corresponding to the third data point of the fitted Hiroshima dose-response curves of Fig. 1) were 0.5 to 1.6 times the reference value. Neutron dose-response curves were then derived for various tilts and are shown in Fig. 16. They suggest an upper limit of gamma-ray tilt not greatly exceeding 1.2 times the reference value. Neutron RBE is only weakly dependent on Hiroshima gamma-ray tilt, the effect of which is similar to that of Hiroshima gamma-ray output (compare Figs. 13 and 16).

Effects on Gamma-Ray Risk Coefficient

Nagasaki Gamma-Ray Output. Because the radiation field at Hiroshima was a mixed one, with a significant neutron component (Loewe and Mendelsohn, 1981a; Kerr, 1981; Auxier, 1977), the only reliable basis from the A-bomb data for inferences concerning gamma-ray

effectiveness is the Nagasaki data. (The bone-marrow neutron dose at Magasaki was less than some $0.3 \%$ of the total dose at slant ranges of most interest to radioepidemiology (Loewe and Mendelsohn, 1981a).) Hence, gamma-ray risk coefficients inferred from the Japanese A-bomb experience depend almost solely on the Nagasaki gamma-ray output. Effects of gamma-ray output variations on the dose-response curve are readily visualized from Fig. 17 by allowing values on the dose axis to be fractions and multiples of the reference values. 
Seen in Fig. 17 are the risk coefficients for chromosomal exchanges, arbitrarily calculated for 100 rad (they are lower at lower dose) corresponding to gamma-ray outputs $0.5,1$, and 2 times the reference value. The risk coefficient is simply inversely proportional to the Nagasaki gamma-ray output. It should be noted that the risk coefficient derived with the reference dose values is compatable, within a factor of 2, with experimental results from human lymphocytes irradiated in vitro (Lloyd et al., 1975).

\section{CONCLUSIONS}

The power that dosimetric parameters have relative to one another in affecting calculated neutron RBE is displayed in Fig. 18. A curve for RBE versus relative Nagasaki yield is not shown; it is equally steep as that for relative Hiroshima yield but of opposite slope. The next most powerful parameter after the device yields is the neutron dose at Hiroshima, whether from neutron output or from tilts 1 or 2. Least powerful is Hiroshima gamma-ray dose, whether from gama-ray output or tilt.

To simplify comparisons, the sensitivity of calculated RBE to changes in each of the parameters can be expressed as a "sensitivity ratio," SR:

$$
S R=\frac{\text { percentage change in RBE }}{10 \% \text { change in parameter value }} .
$$

The parameters are ranked, together with their associated SRs in Table 4.

Although this analysis was done by varying one physical parameter at a time, changes in combinations of parameters should have roughly additive effects, as well as roughly additive constraints. For example, increasing the yield of both devices by the same factor will he no effect on RBE, whereas an increment (from the reference vaiue) of $16 \%$ to Hiroshima yield and a decrement of $16 \%$ to Nagasaki yieló should be equivalent to a $32 \%$ increment to Hiroshima yield and would result in a $35 \%$ reduction in neutron RBE (approximately the biologically limiting value for this physical parameter and model endpoint (Fig. 3 )).

While the concept of gamma-ray risk coefficient may not seem to apply to the endpoint used here (chromosomal aberrations) in quite the same way that it does to cancer induction, the effects of dosimetric parameter variation on risk coefficient will be exactly the same for other endpoints, including carcinogenesis. All ganma-ray risk coefficients derived from the A-bomb data will be inversely proportional to the Nagasaki gamma-ray output (and to the Nagasaki yield, which determines the gamma-ray output). 
The purpose of this analysis has been to evaluate the effects that changes in physical dosimetric parameters can have on biomedical inferences. In general, it is found that the radiobiology can be used both qualitatively and quantitatively to help establish reasonable limits on physical parameter values and that the parameters can be ranked according to their power in affecting neutron RBE.

\section{ACKNOWLEDGMENT}

We thank Dr. W.E. Loewe and Mr. E. Mendelsohn for helpful discussions on matters of dosimetry and Dr. D. Moore for statistica? advice.

Work performed under the auspices of the U.S. Department of Energy by the Lawrence Livermore National Laboratory under contract number $W-7405-E N G-48$. 


\section{REFERENCES}

Auxier, J.A., J.S. Cheka, F.F. Haywood, T.D. Jones, and J.H. Thorngate, 1966, Free-Field Radiation-Dose Distributions from the Hiroshima and Nagasaki Bombings, Health Phys. 12: 425-429.

- 1977, Ichiban: Radiation Dosimetry for the Survivors of the $\overline{B o m b}$ ings of Hiroshima and Nagasaki, (ERDA Technical Information Center, Oak Ridge).

Awa, A.A., T. Sofuni, T. Honda, M. Itoh, S. Neriishi, and M. Otake, 1978, Relationship Between the Radiation Dose and Chromosome Aberrations in Atomic Bomb Survivors of Hiroshima and Nagasaki, J. ." Radiat. Res. 19: 126-140.

Jones, T.D., 1977, CHORD Operators for Cell-Survival Models and Insult Assessment to Active Bone Marrow, Radiat Res. 71: 269-283.

Kerr G.D., 1979, Organ Dose Estimates for the Japanese Atomic-Bomb Survivors, Health Phys. 37: 487-508.

- 1981, Review of Dosimetry for the Atomic Bomb Survivors, paper presented at Fourth Symposium on Neutron Dosimetry, Gesellschaft für Strahlen und Umweltforschung, Munich-Neuherberg, June 1-5, 1981.

Lloyd, D.C., R.J. Purrott, G.W. Dolphin, D. Bolton, and A.A. Edwards, 1975, The Relationship Between Chromosome Aberrations and Low LET Radiation Dose to Human Lymphocytes, Int. J. Radiat. Biol. 28: 75-90.

, R.J. Purrott, G.W. Dolphin, and A.A. Edwards, 1976, Chromosome Aberrations Induced in Human Lymphocytes by Neutron Irradiation, Int. J. Radiat. Biol. 29: 169-182.

Loewe W.E. and E. Mendelsohn, 1981a, Revised Dose Estimates at Hiroshima and Nagasaki, Health Phys. 41: 663-666.

and Nagasaki, NucT. Science and Eng. (submitted).

Marcum, J., 1981, House Attenuation Factors for Radiation at Hiroshima and Nagasaki. Report prepared for the Director, Defense Nuclear Agency, (Draft), 15 May 1981.

Milton R.C. and T. Shohoji, 1968, Tentative 1965 Radiation Dose Estimation for Atomic Bomb Survivors, Atomic Bomb Casualty Cormission Report TR-1-68.

Rossi H.H. and C.W. Mays, 1978, Leukemia Risk from Neutrons, Health Phys. 34: 353-360.

- 1980, Comments on the Somatic Effects Section of the BEIR III Report, Radiat. Res. 84 : 395-406. 
TABLE 1. Transmission factors for T65D compared with those estimated for the Reference Doses.

\begin{tabular}{|c|c|c|c|c|c|c|c|c|}
\hline & \multicolumn{4}{|c|}{ Hiroshima } & \multicolumn{4}{|c|}{ Nagasaki } \\
\hline & \multicolumn{2}{|c|}{ T65 Value } & \multicolumn{2}{|c|}{$\begin{array}{c}\text { "Reference }{ }^{n \star \hbar} \\
\text { value }\end{array}$} & \multicolumn{2}{|c|}{ T65 value } & \multicolumn{2}{|c|}{$\begin{array}{l}\text { Reference } \\
\text { value }\end{array}$} \\
\hline & neutrons & $\begin{array}{l}\text { gamma } \\
\text { rays }\end{array}$ & neutrons & $\begin{array}{l}\text { gamin } 3 \\
\text { rays }\end{array}$ & neutrons & $\begin{array}{l}\text { gamma } \\
\text { rays }\end{array}$ & neutrons & $\begin{array}{l}\text { gamma } \\
\text { rays }\end{array}$ \\
\hline Houses & 0.32 & 0.90 & -0.35 & 0.55 & 0.35 & 0.81 & -0.35 & 0.50 \\
\hline Body* & 0.28 & 0.56 & 0.28 & .0.89 & 0.28 & 0.56 & -0.28 & $\sim 0.89$ \\
\hline Total & 0.09 & 0.50 & $\sim 0.10$ & $\sim 0.49$ & 0.10 & 0.45 & $\sim 0.10$ & $\sim 0.45$ \\
\hline
\end{tabular}

* Derived for the reference spectra as described in text.

** Shielding of bone marrow by body tissue. 
TABLE 2. RBE analysis for radiation-induced chromosomal exchanges using the reference doses.

\begin{tabular}{|c|c|c|c|c|c|c|c|c|}
\hline \multicolumn{3}{|c|}{ Hiroshima } & \multicolumn{3}{|c|}{ Nagasaki } & \multicolumn{3}{|c|}{$\begin{array}{l}\text { Residual } \\
\text { Hiroshima }\end{array}$} \\
\hline $\begin{array}{c}\text { Gamma-ray } \\
\text { dose, } \\
\text { rad }\end{array}$ & $\begin{array}{c}\text { Neutron } \\
\text { dose, } \\
\text { rad }\end{array}$ & $\begin{array}{l}\text { Net } \\
\text { effect* }\end{array}$ & $\begin{array}{c}\text { Gamma-ray } \\
\text { dose, } \\
\text { rad }\end{array}$ & $\begin{array}{l}\text { Neutron } \\
\text { dose, } \\
\text { rad }\end{array}$ & $\begin{array}{l}\text { Net } \\
\text { effect* }\end{array}$ & $\begin{array}{c}\text { Gamma-ray } \\
\text { dose, } \\
\text { rad }\end{array}$ & $\begin{array}{l}\text { Neutron } \\
\text { dose, } \\
\text { rad }\end{array}$ & $\begin{array}{c}\text { Net } \\
\text { effect* }\end{array}$ \\
\hline 0 & 0 & 0 & 0 & 0 & 0 & 0 & 0 & 0 \\
\hline 32 & 0.3 & 1.3 & 32 & 0.05 & 0.1 & 0 & 0.25 & 1.2 \\
\hline 96 & 1.7 & 3.3 & 96 & 0.25 & 1.1 & 0 & 1.45 & 2.2 \\
\hline 152 & 3.4 & 7.5 & 152 & 0.56 & 3.0 & 0 & 2.84 & 4.5 \\
\hline
\end{tabular}

* Percentage of cells with chromosomal exchanges; control values ( $y$-intercepts of fitted curves) subtracted. 
TABLE 3. Effect of neutron Tilt-2 on RBE.

\begin{tabular}{lcc}
\hline $\begin{array}{c}\text { Relative } \\
\text { tilt-2 }\end{array}$ & $\begin{array}{c}\text { Neutron RBE } \\
\text { at } 1 \text { rad }\end{array}$ & $\begin{array}{c}\text { Relative neutron } \\
\text { output }\end{array}$ \\
\hline 0.5 & 154 & 200 \\
0.8 & 130 & 6 \\
1.0 & 111 & 1 \\
1.3 & 102 & .06 \\
2.0 & 92 & .004 \\
\hline
\end{tabular}

TABLE 4. Relative Sensitivity of Neutron RBE to Changes in Physical Parameters.

Physical parameter

$S R *$

Hiroshima device yield

1.2

Nagasaki device yield $\quad 1.2$

Nagasaki gamma-ray output $\quad 1.2$

Hiroshima neutron output $\quad 0.7$

Hiroshima neutron tilt-1 $\quad 0.6$

Hiroshima neutron tilt-2 0.6

Hiroshima gamma-ray output $\quad 0.4$

Hiroshima gama-ray tiit $\quad 0.4$

* Sensitivity Ratio (defined in text). 


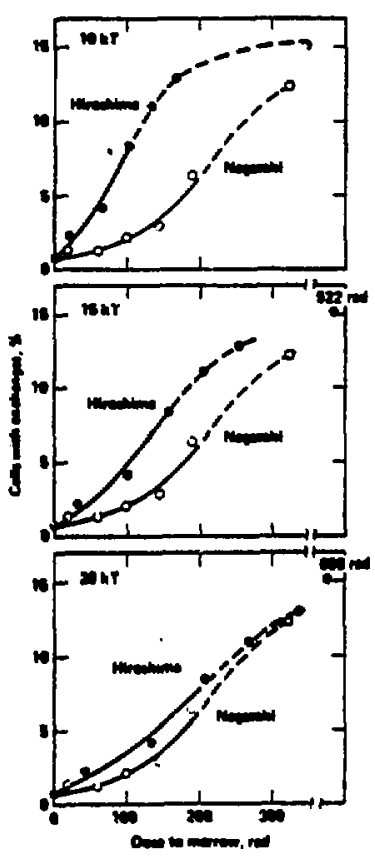

18. 1. Effect on the Hiroshtan dose-response curve, and on inter-city differences, of varying the Hirceiniea device yield.

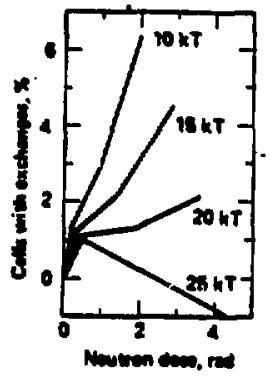

HE. 2. Meutron doseresponce curves for vartoue Hirouhine device yields.

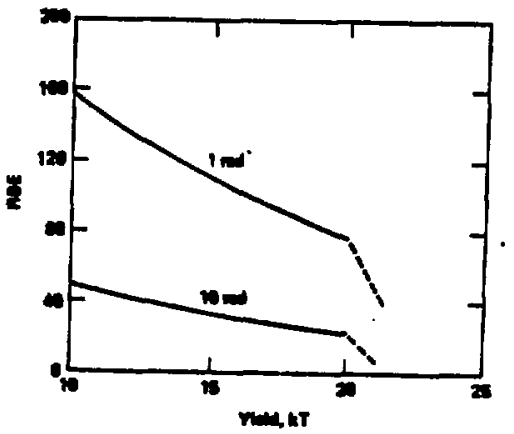

F18. 3. Heutron REE a a function of Hiroshied device yleld, shown for neutron doses of 1 and 10 rad. Dashed portions of curves represent device yleids giving negerlve neutron dose-response functiona. 
F1g. 4. Neutron RDEs derlved for varfous Hiroehlma device yields: comparison with laboratory experiaental values.
F18. 5. Keutron REEa dertvi: for various Magagak1 device yields: compariton with Iaboratory experimental values.
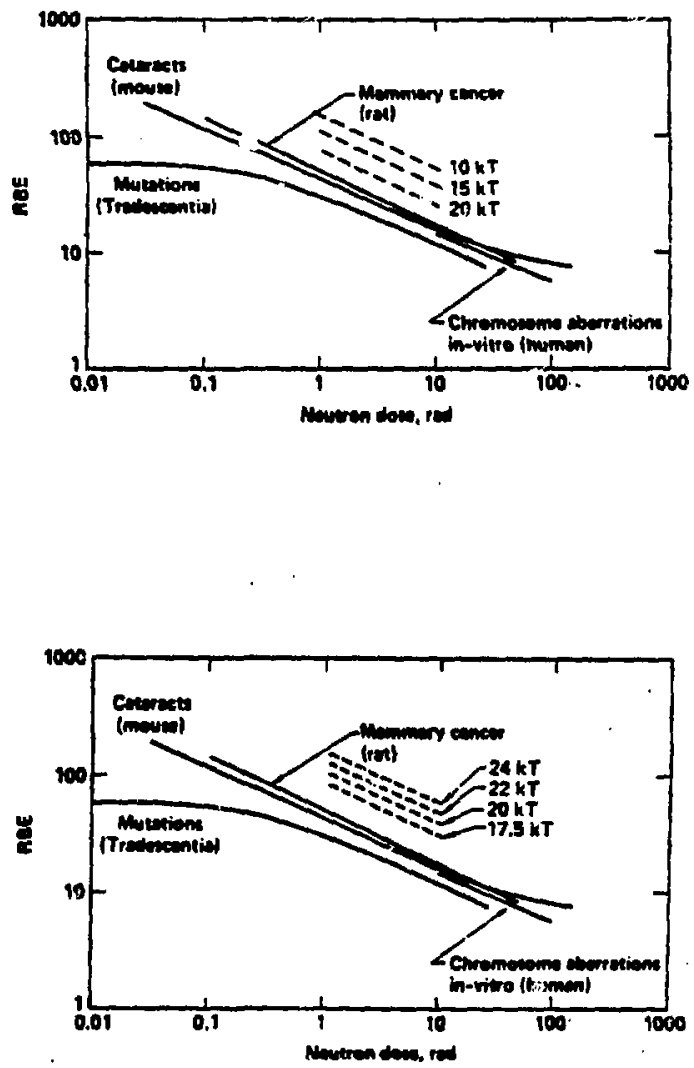

F15. 6. Effect of relative Hiroshine neutrod output or neutron dose-responie.

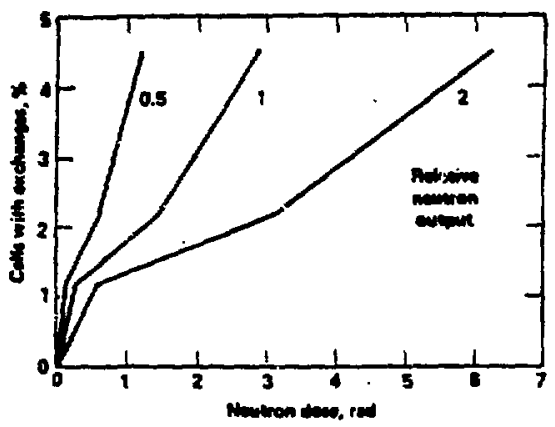


F18. 7. Heutron RBE a a function of relative neutron output, show for neutron doese of 1 and 10 rad.

F18 - 8. Neution RBEa derived for various relative Hiroshime neutron outputs: coperivon with laboratory experiuental data.
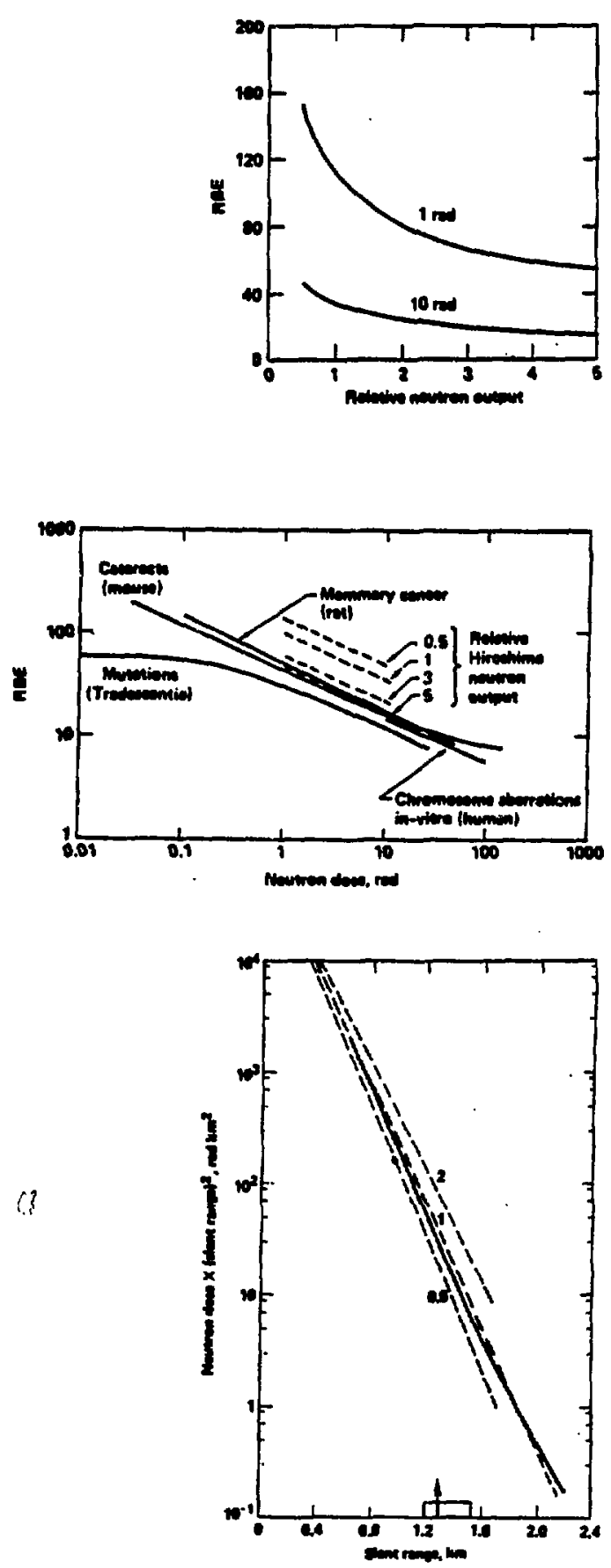

F1t. 9. T11t-1. The solid Ine is the referepce function for UIroshine . lioe 1 (dathed) is a Ineser approxibation to It; 1 ine: 0.5 and 2 represent one helf and twice the reference dose at $1.28 \mathrm{k}$ (arrow). The sheded area between 1.19 and 1.54 kn indicsted the range apan ueed in the present anclsats. 
F1. 10. Effect of relative Biroshime neutron t11t-1 on neutron done-reuponue.

18. 11. Comparison of the effecta of relative

Hirosh1we neutron t11t-1 and neutron output on neutron dose-response.
718. 12. T11t-2, The solid I1ne ia the reference function for Hifoshles. Line 1 (dantiat) is a linear approxineticy to It; linas 0.5 and 2 represent one half and twice the reference dose at $1.54 \mathrm{kn}$ (arrow). The shaded aree between 1.19 and $1.54 \mathrm{~km}$ Indfeates the range span used in the present analyais.
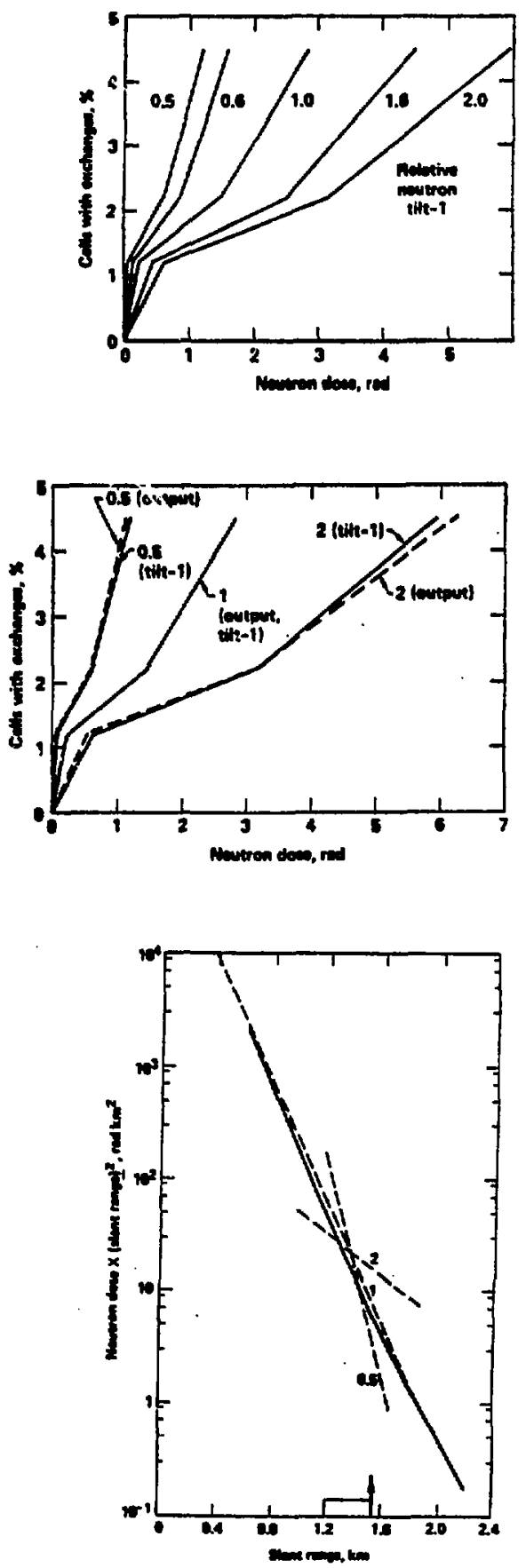
F18. 13. Effect of relative Hiroshime game-ray output on neutron done-renponse.
E18. 14. Neutron REE as a function of Hirouhie. gama-ray output, shown for neutron dosen of 1 and 10 rad. Danhed portions of curves represent gaane-tay outpute giving negative neutron dose-response functions.
F18- 15. Heutron RBE: derived for varioun relative Hiroshina game-ray outputs: couparison ufth liboratory experimental data.
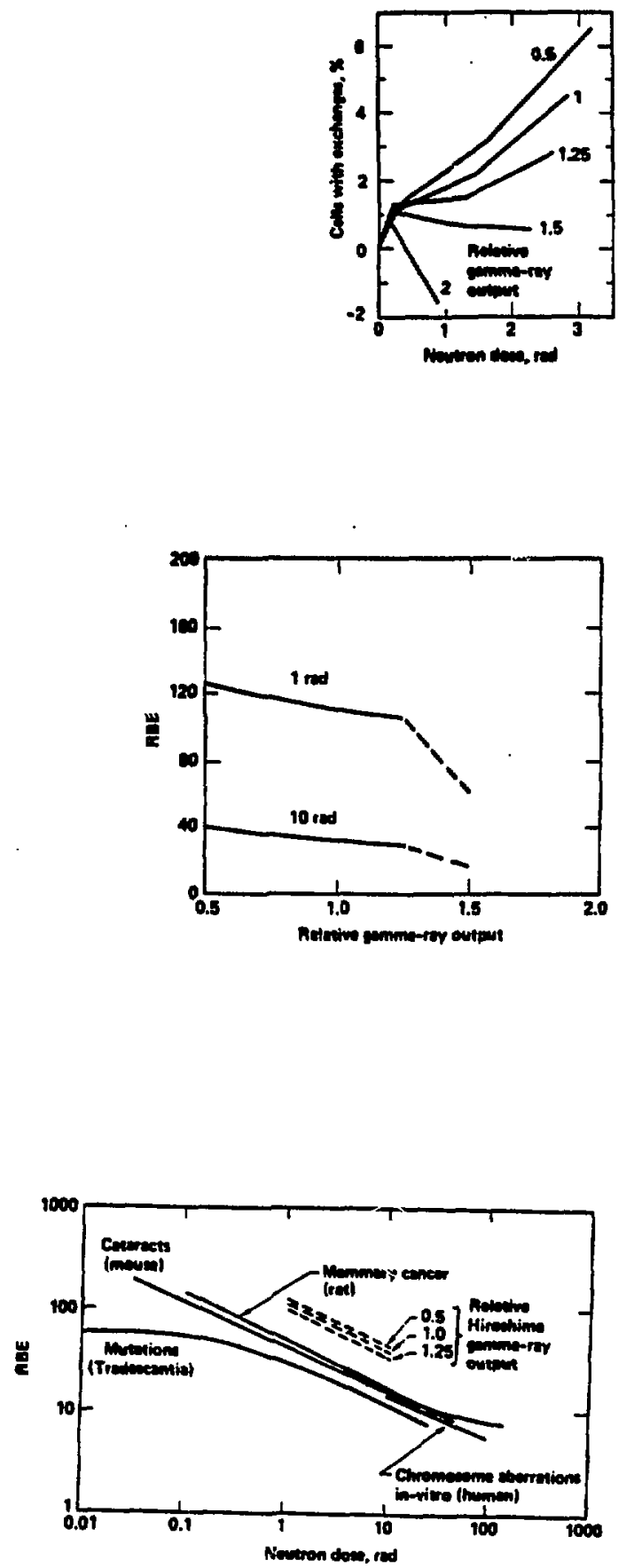
718. 16. Effect of relative Hiroshian guma-ray tilt on neutron dose-response.

15. 17. Effect of relative Nasasaki ganasaray output on the gasan-ray risk coefficleat for chromosonal exchanges. The relative outputs represent 0.5 and 2 tiaes the reference value.
F18. 18. A componte of Hi roshino done-paraweter effect: on neutron RBE. (A relative value of 2.0 is equivalent to the reference value.)
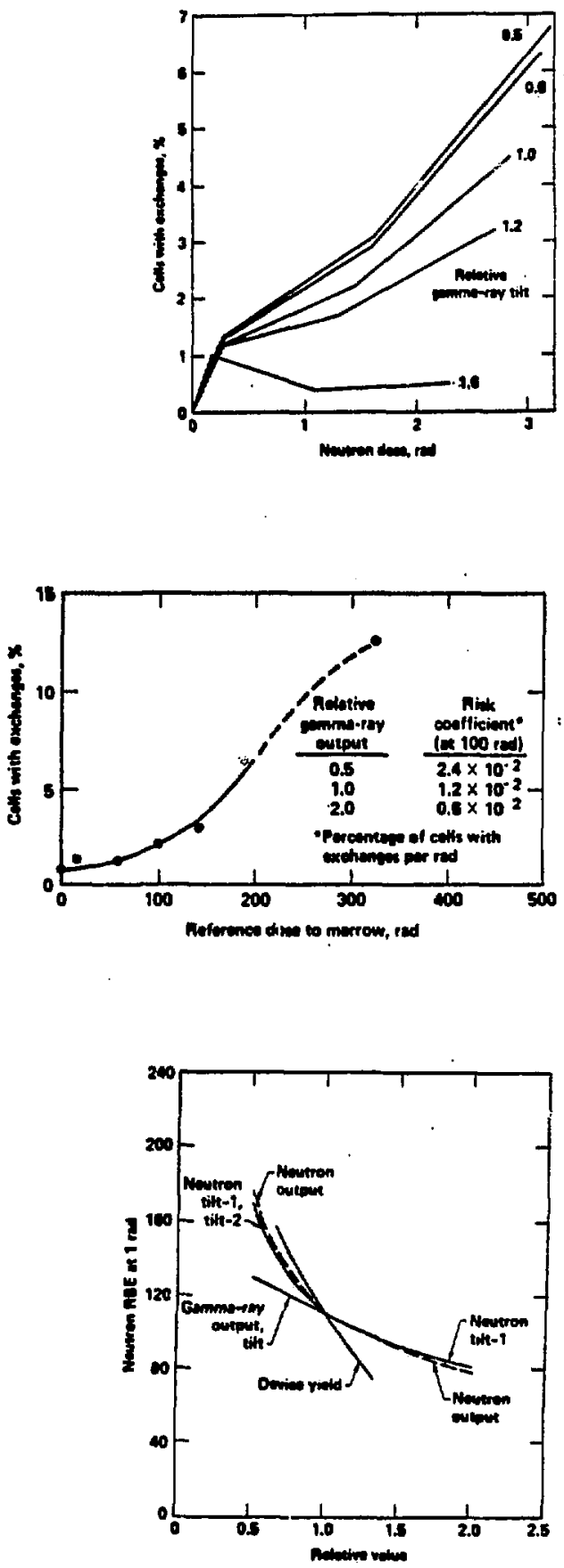
APPENDIX 3

CANCER RISKS AND NEUTRON RBE'S FROM HIROSHIMA AND NAGASAKI

\author{
R. Lowry Dobson and Tore Straume \\ Biomedical. Sciences Division and Hazards Control Department \\ Lawrence Livermore National Laboratory \\ University of California \\ Livermore, CA 94550, USA
}

\begin{abstract}
The new radiation dose estimates for Hiroshima and Nagasaki are here combined with epidemiologic data from the A-bomb survivors and examined radiobiologically for compatability with other human and experimental data. The new doses show certain improvements over the original T65 doses. However, they suggest for chronic granulocytic leukemia, total malignancies, and chromosome aberrations, at neutron doses of $1 \mathrm{rad}$, RBEs in excess of 100 , higher than expected from other findings. This and other indications suggest that either there are unrecognized systematic problems with the various radiobiological data, or the new doses are deficient in neutrons for Hiroshima, by a factor of about five. If in fact there were actually some 5-fold more dose from neutrons at Hiroshima than estimated by the new calculations, the RBEs would agree we 11 with laboratory results, and other inconsistencies would largely disappear. Cancer risks are estimated for neutrons from the new doses and are compared with those estimated from "radiobiologically reconciled" doses (the new doses adjusted by adding approximately 5-fold more neutrons). The latter estimates appear more reasonable. For low-LET radiation, cancer risk estimates are altered very little by the new dose estimates for Nagasaki.
\end{abstract}

\title{
INTRODUCTION
}

New radiation dose estimates for Hiroshima and Nagasaki have recently been proposed $(7,2,3,4,5)$. They differ substantially from the T65 doses $(6,7)$ that for many years have been widely accepted. This is forcing re-examination of radiation risk estimation from the Japanese data. Further dosimetric studies are currently underway at a number of institutions (see reference 8 ), primarily using computational methods; but key experiments, such as energy-spectrum determinations for leakage neutrons from a Hiroshima device mock-up, are planned as well. It should be noted, however, that rather close agreement exists among investigators involved in the new dosimetry. 
The Loewe-Mendelsohn (LM) estimates $(1,2,3)$, on the one hand, and the Kerr $(K)$ estimates $(4,5)$, on the other, are basically similar. Both use Hiroshima radiation output values calculated in 1976 at Los Alamos National Laboratory (9) and employ essentially the same computer code for calculating radiation transport through distance. Their estimates of neutron dose at Hiroshima, the item most changed from the original T65 estimates, are close together. Kerr, however, takes $12.5 \mathrm{kilotons}$ as the yield of the Hiroshima device, while the LM estimate uses $15 \mathrm{kilotons.} \mathrm{A} \mathrm{representative} \mathrm{comparison} \mathrm{of} \mathrm{the}$ three dose est imates is shown in Fig. 1. It is important to note that the neutron component in Hiroshima, though substantially smaller in the new estimates than in T65, has by no means vanished.

When these surprising new dose estimates appeared (1), we first tested them for radiobiological credibility. Our preliminary analyses (10) indicated that the LM doses were generally credible and showed some radiobiological inprovements over T65 doses. We derived cancer risk coefficients and neutron RBEs from them, and recognized that the RBEs (calculated by the method of Rossi and Mays (11)) for certain major biological endpoints appeared somewhat high compared with radiobiological expectations. Next, we made a systematic examination -- quite independent of any particular dose estimates -of the extent to which radiobiological inferences from

Hiroshima-Nagasaki epidemiologic data would be affected by changes in various dosimetric: parameters. Device yield, neutron output, gamma-ray output, and other influencing factors were al lowed to vary over wide ranges, and the radiobiological impact on an indicator endpoint (chromosome aberrations) was determined. A consistent picture was obtained (12), but again the suggestion emerged that the new doses perhaps underestimated Hiroshima neutrons, by a factor of about 5 . This is shown in Fig. 2 .

Here we present further radiobiological comparisons, which continue to raise questions concerning neutrons at Hiroshima, and lead to the notion of "radiobiologicaily reconciled" (RR) dose estimates as a temporary, exploratory approach, which may possibly assist the physical dosimetric search for the best achievable estimates of the doses actually delivered.

\section{LEUKEMIA}

In our earlier preliminary analyses (10), all forins of leukemia were combined and considered together. This was done because Loewe and Mendelsohn (2), as well as Rossi and Mays (11), had used combined data, and we wished our results, which differed from theirs, to be readily comparable. Our differences from Loewe and Mendelsohn (2) stem from two factors: we used more recent epidemiologic data and, importantly, we did not combine data points. The resulting dose-response curve, clearly sigmoidal in shape, as seen in Fig. 3, is radiobiologically more informative, and more compatible with experimental animal results (13). 
It was realized, however, that chronic granulocytic leukeinia (CGL) and acute leukemia (AL) would likely show contrasting responses, as had earlier been noted (with T65 doses) by Mole (14), and more recently by Ishimaru et al. (15). We have separataly analyzed CGL and AL data (15), Using the LM dose estimates. The results, presented in Figs. 4 and 5, are of great interest. CGL, which has a well known association with chromosome abnormality (the Philadelphia chromosome), shows responses that. differ for the two cities (Mantel-Haenszel test, $p<0.05$ ), and neutron RBE values (discussed below) that are very similar to those for radiation-induced chromosome aberrations (10). AL, on the other hand, shows no significant inter-city difference, and neutron RBE of. unity. Thus, AL and breast cancer (10) appear to fall together into one category, with neutrons no more etiologically effective than gamma rays, while CGL falls into another (much larger) category which includes chromosome aberrations and most other malignancies, where neutrons, especially at low doses, are very much more effective than gamma rays (see Fig. 5 in (10)). These observations have importance not only in establishing dose-response relationships, but in suggesting insights into mechanisms of causation.

\section{COMPARISONS WITH CRITICALITY ACCIDENT DATA}

Another approach we have explored is the comparison of cytogenetic findings from nuclear criticality accident victims with Hiroshima-Nagasaki data. This is exceedingly important, as criticality accidents are one of the few other sources of human whole-body neutron exposure experience available. We examined the extremely interesting cytogenetic data obtained by Littlefield and Joiner (16) from six individuals exposed 16 and 17 years earlier to various substantial doses of fission neutrons and gamma rays in the Y-12 accident of 1958 at Oak Ridge. Those investigators had already compared their results with Awa's data (17) for Hiroshima, using T65 doses, and found good agreement. We compared the same $Y-12$ data with newer results of S.wa et al. (18), using the LM Hiroshima dose estimates and, as seen by the Hiroshima curve in Fig. 6, again find remarkably good agreement -- which, however, in this case, with the much reduced neutron component estimated for Hiroshima (see Fig. 1), is unexpected and paradoxical. Neutrons are known (from blood activation data and mock-up measurements (19)) to have contributed some $26 \%$ of the tissue kerma dose at $Y-12$, but are now estinated $(2,4)$ to have contributed only about 2-6\% (depending on distance from the hypocenter) at Hiroshima. Therefore, the $\gamma-12$ data should not coincide with the Hiroshima curve, unless for chromosomal-exchange induction neutrons have RBEs close to unity, which is known not to be the case. Human in vitro lymphocyte data $(20-22)$ and human in vivo data $(18,10,12)$, as well as studies on other material, indicate neutron RBEs greater than 1 , which rise to substantiai values as dose diminishes. However, if perchance the new Hiroshima doses $(2,4)$ do underestimate the neutron component by about 5-fold, that is, if at Hiroshima there actually were 5-times more neutrons than recently 
estimated, then the observed concordance of $\gamma-12$ and Hiroshima curves seen in Fig. 6 would be expected and the paradox solved.

\section{COMPARISONS WITH IN VITRO DATA}

We have examined this in another way. Since in vitro and in $\checkmark$ ivo cose-response relations for radiation-induced chromosome aberrations in human lymphocytes are considered to be essentially identical $(23,24)$, we took experimentally determined in vitro standard curves for gamma rays (21) and for neutrons (20), adjusted them for disappearance of unstable lesions with time (25), and obtained exchange frequencies in blood 7 mmphocytes that would be expected in individuals exposed 23 years earlier (Awa collected his Hiroshima data in 1968-69). These curves are also shown in Fig. 6 . Next, we constructed from them the dose-response curve that would be expected for Hiroshima A-bomb survivors if they had received the gamma-ray doses and neutron doses of the LM estimates (2). The resulting curve is shown in Fig. 6 as a dot-dash line. It does not coincide well with that for observations at Hiroshima; rather, it lies. closer to the Nagasaki observations. If, however, 5-fold more neutrons are assumed for Hiroshima than estimated in (2), the dot-dash curve moves into concordance with that for Hiroshima observations. Thus, the suggestion becomes even stronger, and from independent sources, that neutrons at Hiroshima, though possibly less than estimated in the T65 doses, likely contributed more dose than recently estimated by computations $(2,4)$.

\section{RECONCILIATIONS}

Perspective is gained from intercomparing Figs. 6,7 , and 8 , where the Hiroshima and Nagasak $i$ chromosome data are displayed separately for $L M, T 65$, and $K$ dose estimates. Appearing in each of these figures are the $\gamma-12$ data, so that their relationship to the differing Hiroshima curves can be seen, and also the in-vitro-derived gamma-ray and neutron curves, so that their relationship to the Nagasaki and Hiroshima curves can be seen. With the T65 doses (Fig. 7), the Nagasaki and in vitro gama-ray curves correspond poorly. The Hiroshima curve, however, corresponds quite closely with the curve (not shown) constructed from T65 Hiroshima neutron-gama dose mixtures and in vitro relationships. In contrast, with $k$ doses (Fig. 8) there is excellent correspondence between the Nagaiaki and in yitro gama-ray curves, but the curve (not shown) constructed with K Hiroshima neutron-ganma dose mixtures corresponds poorly with Hiroshima observations (as was the case also for $L M$ doses, discussed above and shown in Fig. 6). A7so, with the LM doses (Fig. 6) correspondence between Nagasaki and in vitro garma-ray curves is remarkably close. Indeed, this close correspondence lends support to the validity of the present reconciliation approach. Similarly, support involving neutron data comes from the almost exact agreement found between $Y-12$ observations and the curve (not shoum) constructed from the in vitro relationships and knowledge that $\gamma-12$ doses (tissue 
kerma) were $26 \%$ neutrons. It should be emphasized that comparisons between $Y-12$ data and constructed Hiroshima curves show good correspondence for T65 doses, but poor correspondence for both $\mathrm{K}$ doses and LM doses.

As previously noted, however, the LM estimates appear some 5-fold low in Hiroshima neutron dose. It is possible, of course, that this effect is due to inadequately understood features of the biological data. An explanation may yet be found there. It does not appear possible, however, to account for this effect on the basis of uncertainties regarding dose transmission factors for houses and the human body (12). An obvious suggestive explanation (which can be tested) is that there were really more neutrons at Hiroshima than the recent recalculations disclose. If this is assumed to be the case, one may add approximately 5-fold more neutron dose (taking into account systematic variations with distance from hypocenter) and arrive at what may be called "radiobiologically reconciled" (RR) dose estimates. Such unifying "RR" estimates satisfy at once the cytogenet ic data from Hiroshima-Nagasaki, $Y-12$, and in vitro sources, and bring neutron RBES for CGL, total malignancies, and chromosome aberrations into agreement with experimental results.

Using "RR" dose estimates -- which are exploratory, strictly tentative, and employed here only as probes while physical dosimetry for Hiroshima is being more thoroughly studied -- we derived (by the Rossi-Mays (11) method) neutron dose-response curves for CGL, tutal malignancies, and chromosomal exchange aberrations. Neutron risk estimates, from the slopes of these (linear) curves, are given in Table 1, together with the (higher) estimates from the unadjusted LM doses. It is possible, of course, that neutron cancer risks actually are as high as those suggested by the LM doses (Table 1), but the radiobiological evidence outlined above suggests that the estimates based on "RR" doses are more likely.

RR-derived neutron RBES are shown in Fig. 9, compared with those based on $L M$ doses and also with laboratory experimental results. The RBEs fall into two categories. On the one hanc, there are those with values near unity (e.g., acute leukemia and breast cancer) that appear not to vary significantly with dose, and on the other hand, those that tend toward high values at low doses (e.g., CGL, total malignancy mortality, and chromosome aberrations). It is clear from Fig. 9 that for the 1 atter category, RBE values derived from $L M$ doses are too high to be consistent with representative experimental radiobiological data, whereas RBEs derived from "RR" doses show rather good agreement with laboratory results.

Cancer risk estimates for low-LET radiation based on bomb-survivor data are altered little by the new doses (10). Values for various malignancies are given in Table 2 for absorbed doses of $100 \mathrm{rad}$ (at 10 rad they are substantially lower). The number 2.8 listed in Table 2 for total leukemia is an overestimate. It was derived from combined data from bcth cities and therefore includes 
neutron-induced CGL of Hiroshima. The number is included here for completeness and comparison.

In conclusion, the suggestion is made that "RR" dose estimates for Hiroshima, such as we have developed here from the newly computed estimates $(2,4)$ together with other relevant radiobiological data, may serve two important functions:

- By identifying radiobiological "inconsistencies", they may significantly aid the extremely important efforts now underway to physically establish what the doses delivered at Hiroshima and Nagasaki actually were.

- They offer an alternative basis (possibly the soundest available at present) for drawing radiobiological inferences from hiroshima-Nagasaki epidemiologic data.

ACKNOWLEDGEMENT

We thank Dr. D. Moore for Statistical assistance. Work performed under the auspices of the U.S. Department of Energy by the Lawrence Livermore National Laboratory under contract number W-7405-Eng-48. 


\section{REFERENCES}

1. Loewe, W.E. and Mendelsohn, E., Revised Estimates of Dose at Hiroshima and Nagasak $i$, and Possible consequences for Radiation-Induced Leukemia, Lawrence Livermore National Laboratory Document 0-80-T4 (1980).

2. Loewe, W.E. and Mendelsohn, E., "Revised Dose Estimates at Hiroshima and Nagasakt," Health Phys. 41, 663-666 (1981).

3. Loewe, W.E. and Mendelsohn, E., "Neutron and Gamma Doses at Hiroshima and Nagasaki," Nuc 1. Sci. Eng. (in press).

4. Kerr, G.D., "Review of Dosimetrj for the Atomic Bomb Survivors," in: Proceedings of the Fourth Symposium on Neutron Dosimetry, Commission of the Eurspean Comunities, Luxembourg (1981) Vol. 1, pp. 501-513.

5. Kerr, G.D., "Findings of a Recent ORNL Review of Dosimetry for the Japanese Atomic-Bomb Survivors," in: Reevalutions of Dosimetric Factors, Hiroshima and Nagasaki, Thiessen, J.W. and Bond, V.P., Eds. DOE Symposiunı Series, CONF-810928, National Technical Information Service, Springfield, Virginia (in press).

6. Auxier, J.A., Cheka, J.S, Haywood, F.F., Jones, T.D. and Thorngate, J.H., "Free-Field Radiation-Dose Distributions from the Hiroshima and Nagasaki Bombings," Health Phys. 12, 425-429 (1966).

7. Auxier, J.A., Ichiban: Radiation Dosimetry for the Survivors of the Bombings of Hiroshima and Nagasaki, ERDA Technical Iliforma.ion Center, Oak Ridge (1977).

8. Reevaluations of Dosimetric Factors, Hiroshima and Nagasaki, Thiessen, J.W. and Bond, V.P , EdS. DOE Symposium Series, CONF-810928, National Technical Information Service, Springfield, Virginia (in press).

9. Henderson, M. et al., Output Calculations and Related Activities Funded by the Defense Nuclear Agency 1975-1978 (U), Los Alamos National Laboratory Document LA-8393-MS, SECRET RESTRICTED DATA (1980).

10. Str=ume, T. and Dobson, R.L., "Implications of New Hirushima and Nagasakj Dose Estimates: Cancer Risks and Neutron RBE," Health Phys. 41, 666-671 (1981).

11. Rossi, H.H. and Mays, C.W., "Leukemia Risk from Neutrons," Health Phys. 34, 353-360 (1978). 
12. Mendelsohn, M.L., Straume, T., and Dobson, R.L., "Sensitivity of Hiroshima and Nagasaki Epidemiologic Inferences io Dosimetric Parameters," in: Reevaluations of Dosimetric Factors, Hiroshima and Nagasaki, Thiessen, J.W. and Bond, Y.P., Eds. DOE Symposium Series, CoNf-810928, National Technicel Information Service, Springfield, Virginia (in press).

13. Upton, A.C., "The Dose-Response Relation in Radiation-induced Cancer," Cancer Res. 21, 717-729 (1961).

14. Mole, R.H., "Ionizing Radiation as a Carcinogen. Practical Questions, and Academic Pursuits," Brit. J. Radiol. 48, 157-169 (1975).

15. Ishimaru, T., Otake, M., and Ichimaru, M., "Dose-Response Relationship of Neutrons and $\gamma$ Rays to Leukemia Incidence among Atomic Bomb Survivors in Hiroshima and Nagasaki by Type of Leukemia, 1950-1971," Radiat Res. 77, 377-394 (1979).

16. Littlefie1d, L.G. and Joiner, E.E., "Cytogenetic Follow-Up $S$ :udies in Six Radiation Accident Vict ims 16 and 17 Years Post-Exposure," in: Late Biological Effects of Ionizing Radiation, IAEA, Vienna (T978) VoT. 1, pp. 297-308.

17. Awa, A.A., "Chromosome aberrations in Somatic Cells," J. Radiat. Res. Supplement 122-131 (1975).

18. Awa, A.A., Sofuni, T., Honda, T. Itoh, M., Niriishi, S., and Otake, M., "Relationship Between the Radiation Dose and Chromosome Aberrations in Atomic Bomb Survivors of Hiroshima and Nagasaki," J. Radiat. Res. 19, 126-140 (1978).

19. Hurst, G.S., Ritchie, R.H., and Emerson, L.C., "Accidental Radiation Excursion at the Oak Ridge Y-12 Plant -- III Determination of Radiation Doses, "Health Phys. 2, 121-133 (1959).

20. Lloyd, D.C., Purrott, R.J., Dolphin, G.W., and Edwards, A.A.,. "Chromosome Aberrations Induced in Human Lymphocytes by Neutron Irradiation," Int. J. Radiat. Biol. 29, 169-182 (1976).

21. Lloyd, D.C., Purrott, R.S., Dolphin, G.W., Bolton, D., and Edwards, A.A., "Relationship Between Chromosone Aberrations and Low LET Radiation Dose to Humen Lymphocytes," Int. 3. Radiat. Res. 28, 75-90 (1975).

22. Biola, M.T., LeGo, R., Vacca, G., Ducatex, G., Dacher, J., and Bourguignon, M., "E.fficacité relative de divers rayonnements mixtes gamna, neutrons pour l'induction in vitro d'anomalies chromosomiques dans les lymphocytes humains," in: Biological Effects of Heutron Irradiation, IAEA, Vienna (1974) pp. 22T-223. 
23. Randolph, M.L. and Brewen, J.G., "Estimation of Whole-Body Doses by Means of Chromosome Aberrations observed in Survivors of the Hiroshima A-Bomb," Radiat Res. 82, 393-407 (1980).

24. DuFrain, R.J., Littlefield, L.G., Joiner, S.E., and Frome, E.L., "In Vitro Human Cytogenet $1:$ Dose-Response Systerns," in: The Medical Basis for Radiation Accident Preparedness, Hïbner, K.F. and Fry, S.A., E.ds., Elsivier/North Holland, New York (1980) pp. $357-374$.

25. Buckton, K.E., Hamilton, G.E., Paton, L., and Langlands, A.O., "Chromosome Aberrations in Irradiated Ankylosing Spondylitis Patients," in: Mutagen-Induced Chromosome Damage in Man, Evans, H.J. and !.loyd, D.C., Eds., Yale University, New Haven (1978) pp. $14 \hat{c}-150$. 
TABLE 1. Neutron risk coefficients. ${ }^{a}$

Induced effect

LM doses

$R R$ doses

CGL

$\begin{array}{cc}70^{b} & 12^{b} \\ 409^{c} & 86^{c} \\ 1.5^{d} & 0.26^{d}\end{array}$

Total malignancies

Chroniosomal exchanges

$1.5^{\mathrm{d}}$

$0.26^{\mathrm{d}}$

aDetermined for dose to bone marrow.

bcases frer $10^{6}$ person year rad.

CDeaths per : $0^{\text {f }}$ person year rad.

dExchanges per $10^{2}$ cell rad.

TABLE 2. Gamma-ray risk coefficients for

malignantils (for 1su-rad LM absorbed dose).

Pisk
Induced Effect
Coefficient

CGL

$$
.0^{\mathrm{a}}
$$

AL

$1.8^{2}$

Total ieukemia

$2.8^{b}$

Breast cancer

$2.3^{a}$

Total malignancies

$1.6^{\mathrm{b}}$

${ }^{a}$ Cases per $10^{6}$ person year rad.

$b_{\text {Deaths per }} 10^{6}$ person year rad. 


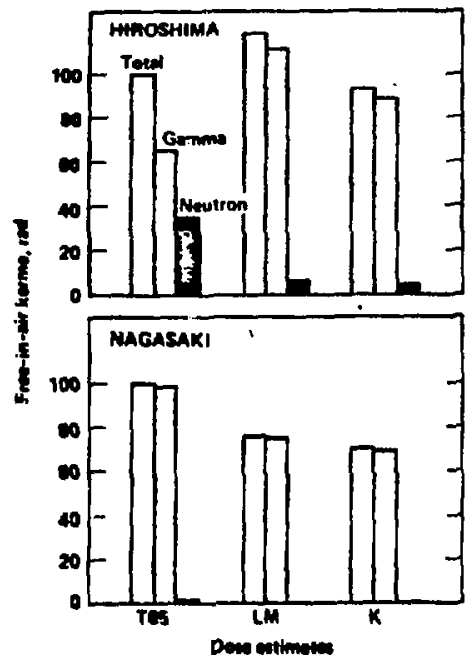

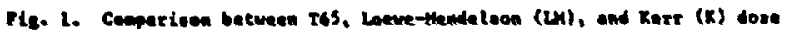

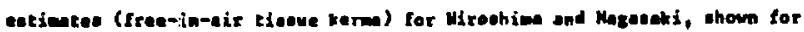
distewee-from-hypocenter in each city $(1,260$ - for Hirouhim, 1,550 a for

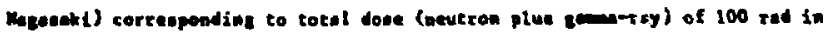
Its entivate.

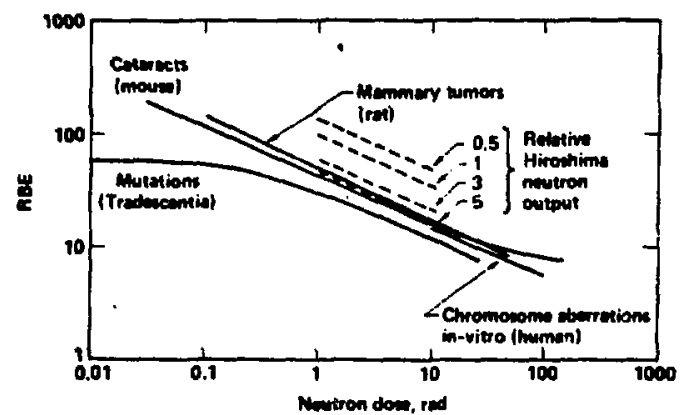

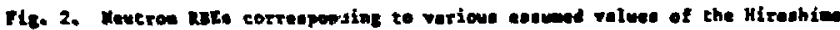

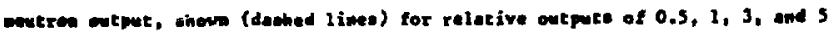

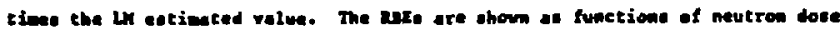

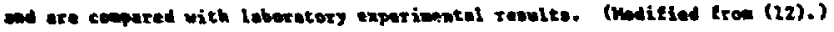




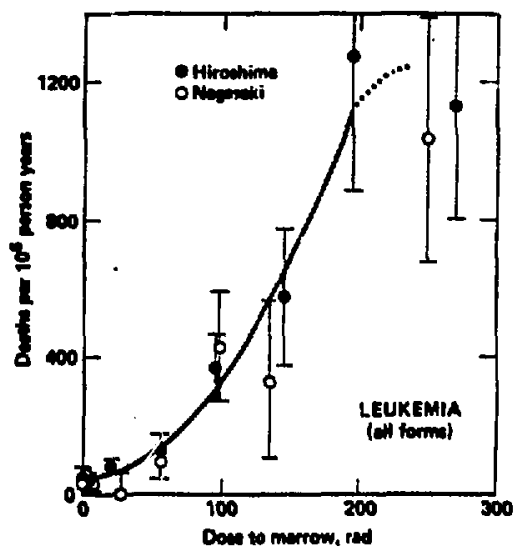

ris. 3. Hortality tron all forn of leukento in the two cities, whom an a

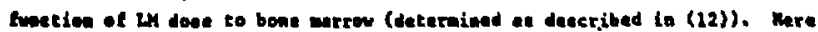
and is Iigu, 4-5, wolid curves are limear-quedratic, leat equares beat fita to two date pointe, with each point wizhted by $1 /(S D)^{2}$. Detted curven axtend beyead the date uned for fiteine and analyalo fdowmard curvature at

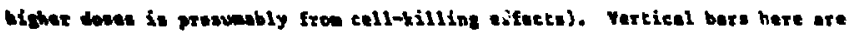
$\pm 150$.

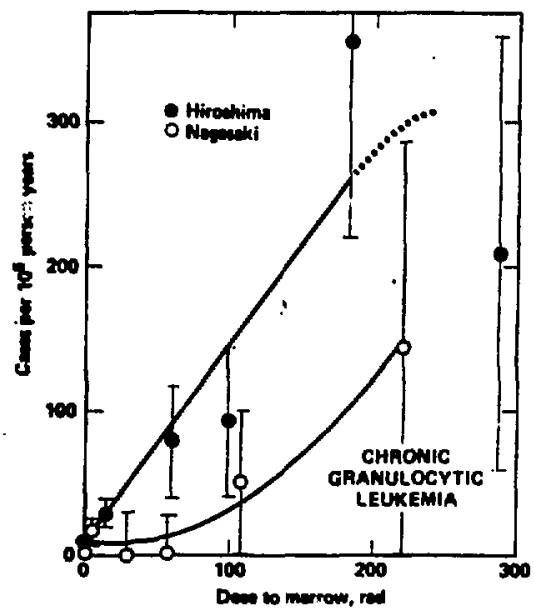

18. 4. Ineidence of ehrenile gremilecytie lenkeaid (COL) in the two citied, chem tor each city a a function of UH doos to bone marrow. Vertfcal bars, \pm 1 s. 


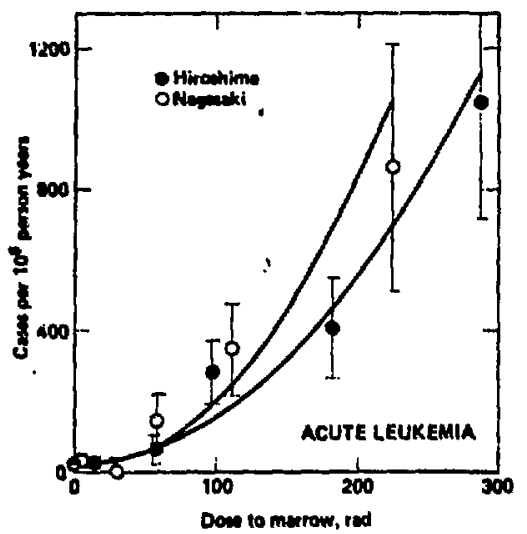

7if. 5. Ineidence of ecute Ievkemila in the two eifies, whow tor each city as - tunction of LA dose to bone warron. Vertical bars, \pm 1 gD.

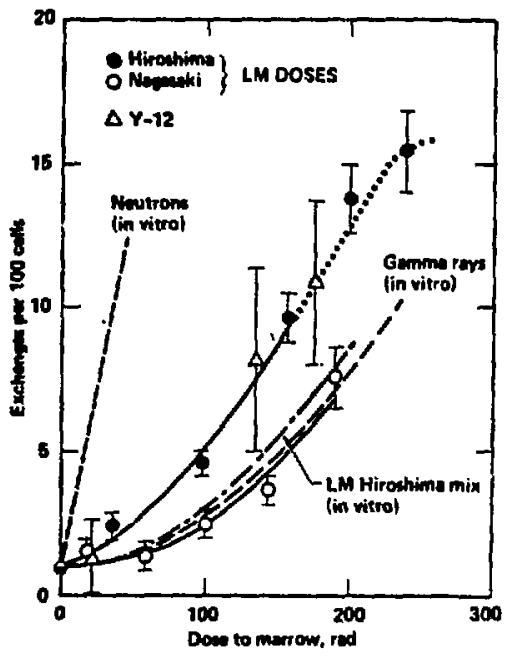

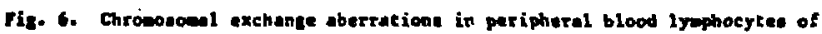
A-bous ourvivore in the two sities and of $\mathrm{Y}-12$ atcitent victien, show as

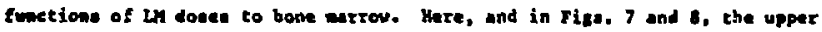
colid curve lo the tit to Mirowhiea data, and che lower solid eurre the fit to Maquaki dete; vertical bare are \pm 2 SD (baued on exchangew/100 ceile and maber of cell. acored). The dached in vitro neutron and gama-ray curven, hore ad in Fige. 7 and 8 , vere darived as described in text. The dot-dash curve is the prediction (from in vitro curven) for Hirouhian vith un nevtron end emonray doese mixtureu. 


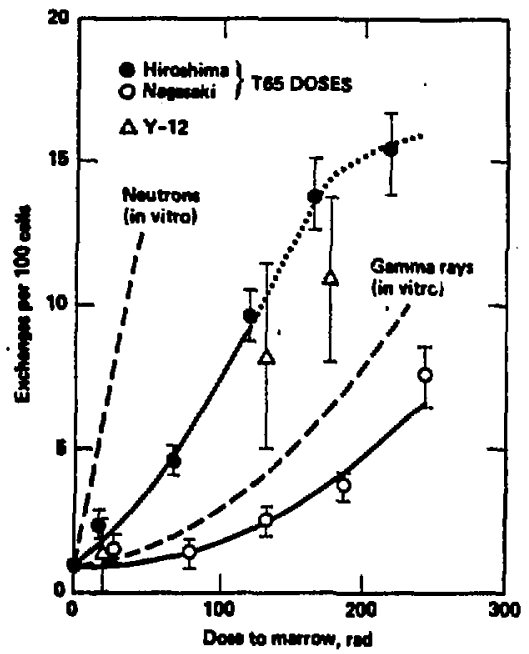

Fis. 7. Chrowoseanl axchange aberrations in blood lyophocytet of A-beab uurvivare and of Y-12 eceident victien, show at tunctione of I65 domes to beme arrou. sea ris. 6 caption.

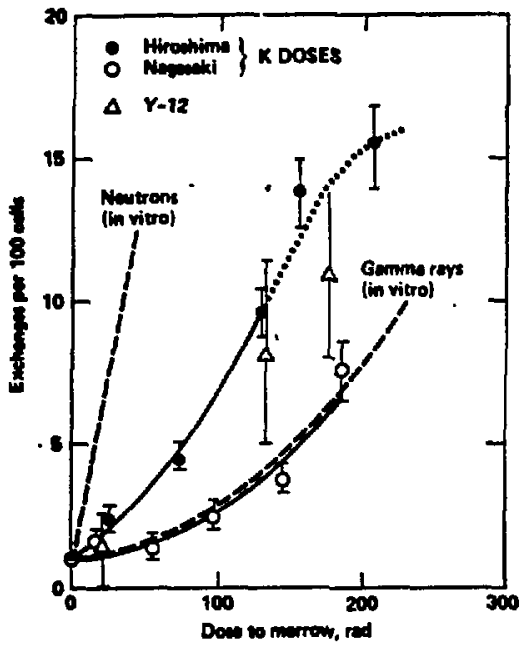

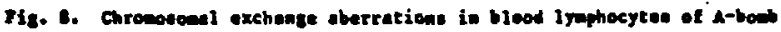
curvivers aud of $\mathrm{Y}-12$ accident victies, show an functione of $\mathrm{X}$ deace to bone martow. See Fir. 6 eaption. 


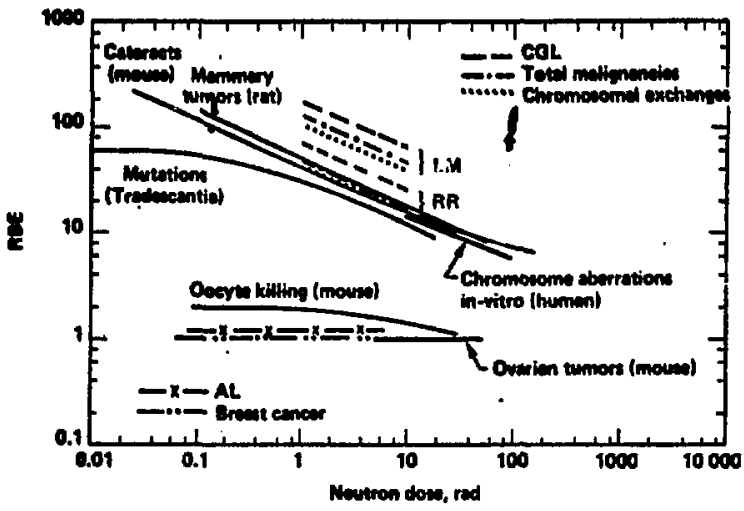

Fic. 9. Mevtron Rate for fira Mirouhima-Mazanaki endpointe (chrenie sremulecytie leukenia, total mlignaney wortality, chrowsomal exchangee, acute leukenia, and breact cancer) derived on the besis of un dares and of

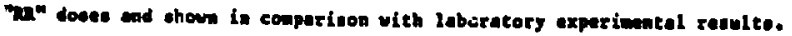

